Argonne National Laboratory 9700 South Cass Avenue

Argonne, Illinois 60439

\title{
HIGH ENERGY PHYSICS DIVISION SEMIANNUAL REPORT OF RESEARCH ACTIVITIES
}

July 1, 1995 - December 31, 1995

Prepared from information gathered and edited by the Committee for Publications and Information:

$\begin{array}{ll}\text { Members: } & \text { J. Norem } \\ & \text { D. Bajt } \\ & \text { R. Rezmer } \\ & \text { R. Wagner }\end{array}$

DISTRIBUTION OF THIS DOCUMENT IS UNEIMITED

October 1996

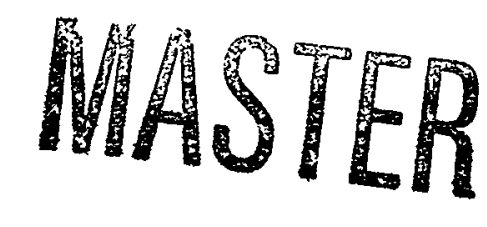




\section{Contents}

I Experimental Research Program $\ldots \ldots \ldots \ldots \ldots \ldots \ldots \ldots \ldots \ldots \ldots 2$

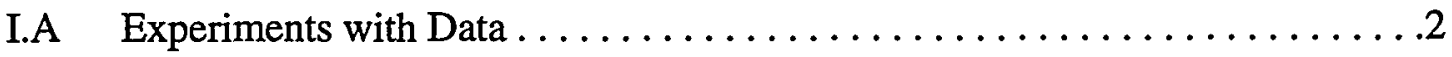

I.A.1 Medium Energy Physics Program .................... 2

I.A.2 Polarized Proton Physics at Fermi National Accelerator Lab ......... 3

I.A.3 Collider Detector at Fermi National Accelerator Lab ........... 4

I.A.4 Non-Accelerator Physics at Soudan .................... 11

I.A.5 ZEUS Detector at HERA. ......................... 20

I.A.6 BNL AGS Partial Snake Magnet Experiment ................ 34

I.B Experiments in Planning or Construction ................... 35

I.B.1 RHIC Spin Physics Program and STAR Detector ............ 35

I.B.2 MINOS-Main Injector Neutrino Oscillation Search .............36

I.B.3 ATLAS Calorimeter Development ....................40

I.C Detector Development ............................ 42

I.C.1 CDF Detector and DAQ Electronics Development ............ 42

I.C.2 ZEUS Detector Upgrades . . . . . . . . . . . . . . . . . . . . 44

I.C.3 STAR Calorimeter Development ..................... 48

I.C.4 ATLAS Calorimeter Design .......................50

I.C.5 ATLAS Detector Research \& Development ...............56

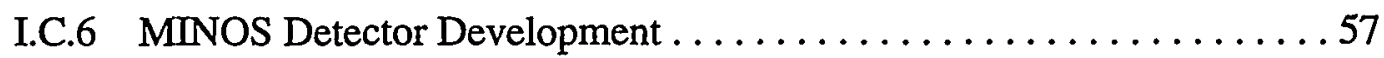

I.C.7 Electronics Support Group . . . . . . . . . . . . . . . . . 61

II Theoretical Physics Program $\ldots \ldots \ldots \ldots \ldots \ldots \ldots \ldots \ldots \ldots \ldots . \ldots \ldots$

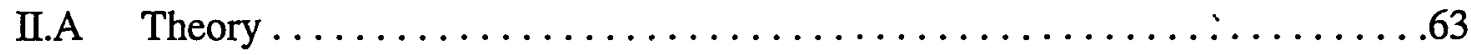

II.A.1 Lattice Formulation of Chiral Gauge Theories . . . . . . . . . . .63

II.A.2 Top Quark Production Dynamics ....................63

II.A.3 t-channel Unitarity Construction of Small-x Kernels . . . . . . . . . . 65

II.A.4 Deep Inelastic Diffractive Scaling . . . . . . . . . . . . . . 65

II.A.5 Isolated Photon Cross Sections . . . . . . . . . . . . . . . . . . . 65 


\section{DISCLAIMER}

Portions of this document may be illegible in electronic image products. Images are produced from the best available original document. 
II.A.6 Double Prompt Photon Production in Polarized Proton-Proton

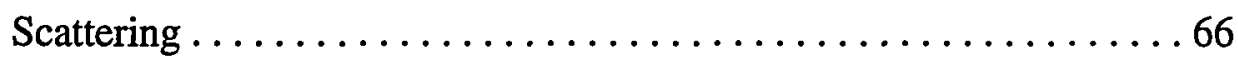

II.A.7 Lattice Measurement of Matrix Elements for Decays of Heavy Quarkonium .............................67

II.A.8 Geometry and Duality in Supersymmetric Sigma-models . . . . . . . 68

II.A.9 Production of a Prompt Photon in Association with a Heavy Quark . . 68

II.A.10 Analysis of Recent Polarization Experiments ...............69 69

II.A.11 Report of the TeV2000 Study Group on Future Electroweak Physics at the Tevatron ......................... 70

II.A.12 High-p $\tau$ Higgs Boson Production at Hadron Colliders to

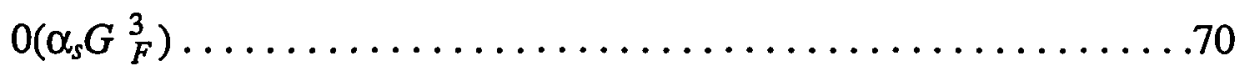

II.A.13 Detecting a Light Stop from Top Decays at the Tevatron ......... 71

II.A.14 Diffractive Deep Inelastic Scattering and Exclusive Vector Meson Production at HERA in Perturbative QCD .................71

II.B - Computational Physics (Lattice Gauge Theory) . . . . . . . . . . . . . 72

III Accelerator Research and Development Program $\ldots \ldots \ldots \ldots \ldots \ldots \ldots 75$

III.A Argonne Wakefield Accelerator Program ..................... 75

III.A.1 AWA Facility Status . . . . . . . . . . . . . . . . . . . . . 75

III.A.2 Experiments and New Ideas . ...................... 77

III.B High Resolution Profile Monitor Development .................78

IV Divisional Computing Activities $\ldots \ldots \ldots \ldots \ldots \ldots \ldots \ldots \ldots \ldots \ldots \ldots ., 81$

IV.A High Performance Computing: The PASS Project $\ldots \ldots \ldots \ldots \ldots \ldots . \ldots 1$

IV.A.1 R\&D During the Period July 95 through December $95 \ldots \ldots \ldots \ldots 81$

V Publications $\ldots \ldots \ldots \ldots \ldots \ldots \ldots \ldots \ldots \ldots \ldots \ldots \ldots \ldots \ldots \ldots \ldots . . \ldots 2$

VI Colloquia and Conference Talks ..........................96 
VII High Energy Physics Community Activities...................99

VIII High Energy Physics Division Research Personnel ...............101 


\begin{abstract}
This report describes the research conducted in the High Energy Physics Division of Argonne National Laboratory during the period July 1, 1995 - December 31, 1995. Topics covered here include experimental and theoretical particle physics, advanced accelerator physics, detector development, and experimental facilities research. Lists of division publications and colloquia are included.
\end{abstract}




\section{EXPERIMENTAL RESEARCH PROGRAM}

\section{A EXPERIMENTS WITH DATA}

\section{A. 1 Medium Energy Physics Program}

The focus of the medium energy polarization program has been on the study of spin effects in proton-proton (pp) and neutron-proton (np) scattering. Combining data from these experiments with measurements from other groups will allow the determination of nucleon-nucleon amplitudes. These amplitudes are needed to understand the strong interaction at intermediate energies, nucleon scattering from nuclei, and the interpretation of some electron scattering experiments at CEBAF.

A major paper describing final results for four np elastic scattering spin observables $\left(\mathrm{C}_{S \mathrm{~S}}, \mathrm{C}_{\mathrm{SL}}=\mathrm{C}_{\mathrm{LS}}, \mathrm{C}_{\mathrm{LL}}, \mathrm{C}_{\mathrm{NN}}\right)$ with a polarized neutron beam incident on a polarized proton target has been accepted for publication in Physical Review D. The full data set from this series of experiments covers beam kinetic energies of $484-788 \mathrm{MeV}$ and c.m. angles from 30 - 180 degrees. These data have a major impact on the isospin- 0 nucleon-nucleon amplitude determinations in this energy range.

Work has continued on a second paper describing LAMPF measurements of $n+p$ $\rightarrow d+$ pi spin observables at 484 and $634 \mathrm{MeV}$. The data analysis is being completed at the University of Montana and Argonne, and all other remaining work will take place at Argonne. Two of the three sets of data have been completely analyzed, and the third set should be finished soon. The goal is to complete and submit this paper by summer. These will be the last nucleon-nucleon data published from our program at LAMPF.

The remaining ANL experimental equipment at LAMPF was packed in a sea container and prepared for shipment. Nearly all the hardware was checked for residual radioactivity, but none was found. Part of the time during the two trips to pack the equipment was spent assisting an experiment (E1178) to measure the polarization parameter for pi- $+\mathrm{p}$ charge exchange scattering at low energies using the Neutral Meson Spectrometer. We helped with the polarized target and the data collection.

Work on the data analysis for the pp experiments at Saclay continued. Data from 1992 runs on 9-track tape were copied to Exabyte $(8 \mathrm{~mm})$ tapes, and many corrupt data files were repaired. A program was written to locate changes in trigger and detector efficiencies, and a large effort was made to analyze all the 1993, 1994, and 1995 runs with this program. Some problems were found that required software changes before the final analysis; these changes have been made. Other problems still require more study. There was a trip to Saclay to discuss the data analysis by C. Allgower and H. Spinka in December 1995. Very useful information on the experiment was obtained during these discussions. It is anticipated that these remaining problems will be solved, and that the final data analysis will be in progress by spring. 
The possibility of joining a collaboration to study baryon spectroscopy at Brookhaven using the SLAC Crystal Ball was discussed with physicists from UCLA, Abilene Christian University, and Brookhaven. These discussions are continuing.

(H. Spinka)

\section{A. 2 Polarized Proton Physics at Fermi National Accelerator Lab}

Recent results on analyzing power measurements in inclusive Lambda production with a $200-\mathrm{GeV} / \mathrm{c}$ polarized proton beam are published in Phys.Rev.Lett. The data indicate a substantial negative $A_{N}$ (about $10 \%$ ) at relatively large $x_{F}$ (above 0.5 ) and moderate $p_{T}$ (above $0.6 \mathrm{GeV} / \mathrm{c}$ ). For $x_{F}$ less than 0.5 or $p_{T}$ less than $0.6, A_{N}$ is compatible with zero. The onset of substantial asymmetry $A_{N}$ in $\Delta$ production further strengthen the case brought up in $\Lambda$ polarization that single-spin effects might be appreciably larger than expected from perturbative QCD.

A paper on "Single-Spin Asymmetries and Invariant Cross Sections of the High Transverse-Momentum Inclusive $\pi^{0}$ Production in 200-GeV/c $p p$ and $\vec{p}$ - $p$ Interactions" is to be published in Phys. Rev. The measured asymmetries are consistent with a value of zero, as shown in Fig. 1, within the error bars.

A paper on "The Difference Between the $p p$ and $\bar{p}-p$ Total Cross Sections with Spins Antiparallel and with Spins Parallel Longitudinally at $200 \mathrm{GeV} / \mathrm{c}$ " is completed and sent it for publication. The cross section of $\Delta \sigma_{L}(p p)$ at $200 \mathrm{GeV} / \mathrm{c}$ is consistent with zero although we observed as large as $-30 \mathrm{mb}$ at lower energies.

The following reactions are investigated:

$\eta(550), \omega(783)$,and $\mathrm{K}$-short (500) production in $p p$ and $\bar{p}-p$ at large $x_{F}$.

$A_{L L}$ in $\pi^{\circ}$ production in $p p$ and $\bar{p}-p$ with charged particles associated.
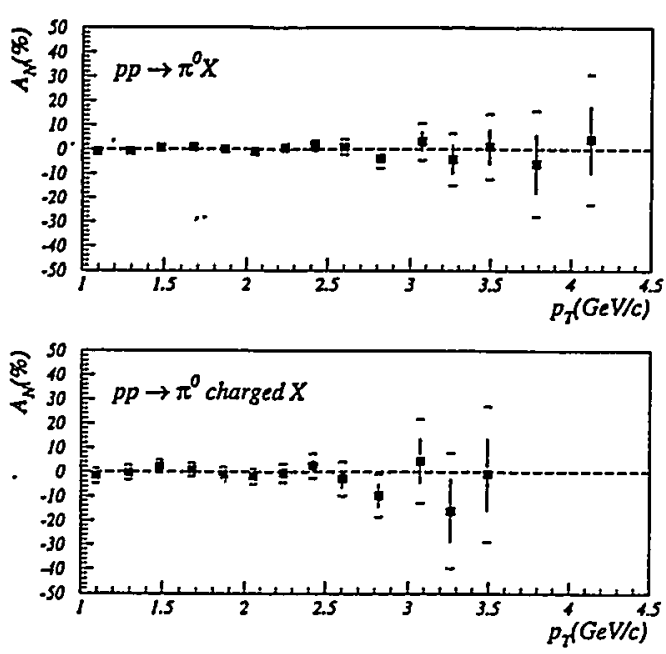

Figure 1. Single spin asymmetry of $\pi^{0}$ and $\pi^{0}+$ charged in $p p$ at $200 \mathrm{GeV} / \mathrm{c}$.

(A. Yokosawa) 


\section{A. 3 Collider Detector at Fermilab}

\section{a. CDF Physics Results}

\section{Top Quark Studies}

Analysis of the full $\sim 110 \mathrm{pb}^{-1}$ sample shows consistency with the partial sample as published. Various systematic studies have been undertaken in order to understand systematic effects, particularly gluon effects, in measuring the top mass using lepton plus jet events. A top signal was isolated in the all hadronic channel, consistent with dilepton and lepton plus jets. Marcus Hohlmann has been working to use $\tau$ lepton signatures in identifying top dilepton candidates.

Tom LeCompte, a new member of our group, working with Rob Roser from Illinois, has derived limits on nonstandard top decays. By looking for photon jet combinations consistent with the top mass along with some other evidence of a second top in the event they have bee able to set a 95\% CL limit on $B(t \rightarrow c \gamma)+B(t \rightarrow u \gamma)$ of $2.9 \%$.

\section{$\underline{\text { W Mass Measurement }}$}

Barry Wicklund developed a method of obtaining a map of conversion points from the inclusive electron sample as a starting point for a more accurate understanding of the material traversed by tracks; the published Run 1a W mass analysis only claimed an accuracy on material in $\mathrm{dE} / \mathrm{dx}$ of $\pm 30 \%$ and this can be improved substantially. Adam Hardman, a Purdue graduate student, has been recruited to work with our group on the W mass using muons. Karen Byrum completed making the Run $1 \mathrm{~b}$ inclusive electron sample available for Larry Nodulman and Karen to come up with the ultimate tower calibration and response trim for the central EM calorimeter, replacing the earlier calibration done by Larry using the $\mathrm{W}$ electron sample. The gain time dependence by arch is shown in Fig. 1. An arch is both a thermal mass and an individual high voltage supply.

\section{$\underline{\text { Vector Boson Couplings }}$}

Bob Wagner has continued to work with colleagues from UCLA, Ilinois and Texas Tech on extending the study of $\mathrm{W} \gamma$ and $\gamma$ production to the full data set including central and plug photons together.

Theresa Fuess along with Chris Wendt from Wisconsin performed a preliminary analysis of the full dataset, extending their limit on high $p_{t} \mathrm{WW}$ and $W Z$ production using events with jet pairs and leptons. A sample nonstandard coupling limit is shown in Fig. 2. Both have subsequently left the field. 


\section{$\underline{\text { High } E_{T}} \underline{\text { Jets }}$}

The excess of jet production at high $E_{T}$ has persisted in CDF data, as systematics continue to be examined. Steve Kuhlmann, with his CTEQ colleagues, have submitted a manuscript on the possible contribution of parton distributions to the high $p_{\mathrm{T}}$ jet excess. An increase in the high- $x$ gluon distribution can largely reconcile the CDF data without contradiction with direct photon data, Fig. 3 , but the comparison with the UA2 inclusive jet data, Fig. 4, is less favorable.

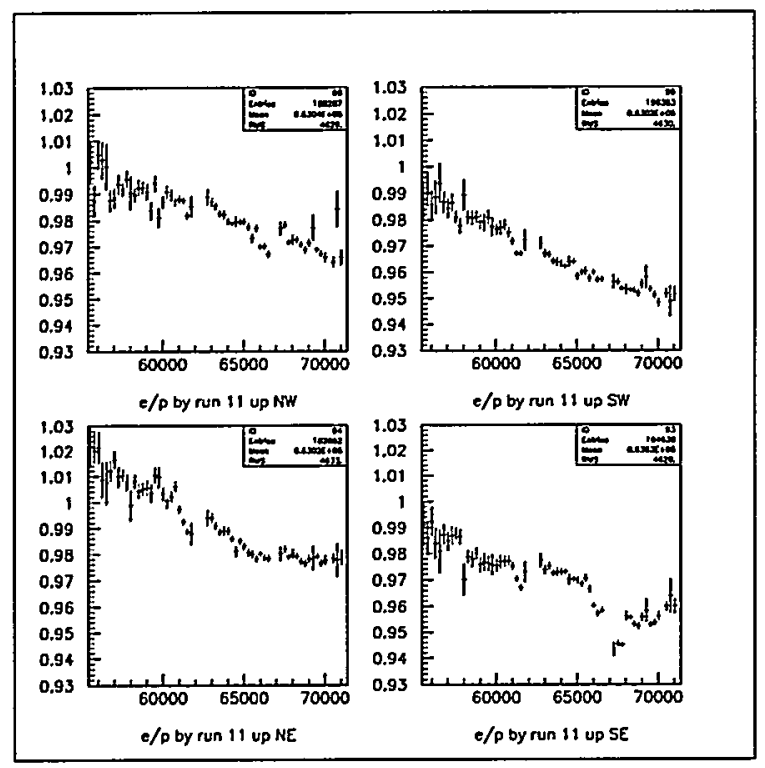

Figure 1: The trend in run number (time) of $E / p$ for inclusive electrons for Run $1 b$, by arch. The apparentdiscontinuity for the SE arch corresponds to a high voltage excursion. 


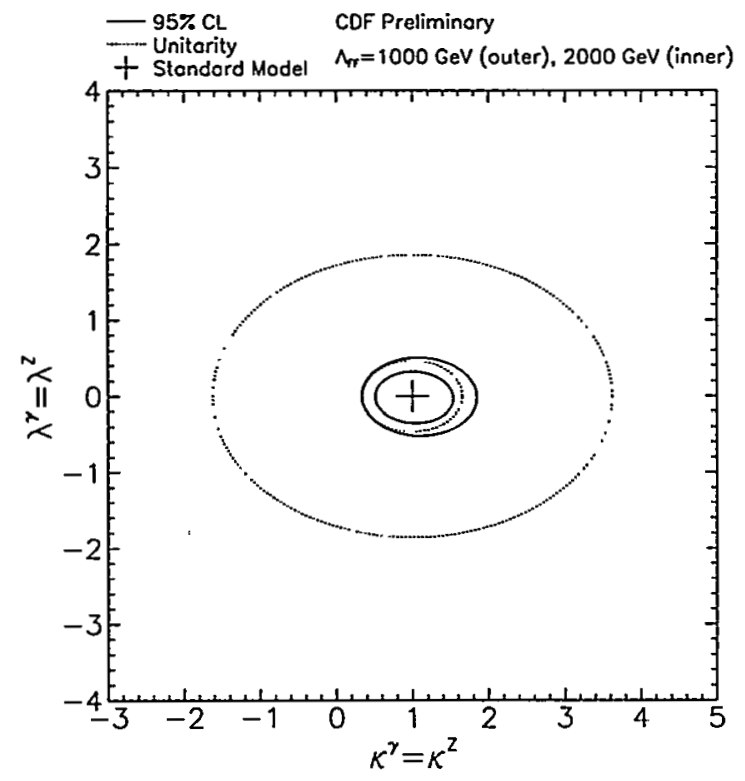

Figure 2: The limits on couplings $\kappa$ (should be 1 ) and $\gamma$ (should be 0 ) assuming $Z$ and $\gamma$ couplings the same. Form factor assumptions are noted as well as unitarity bounds.

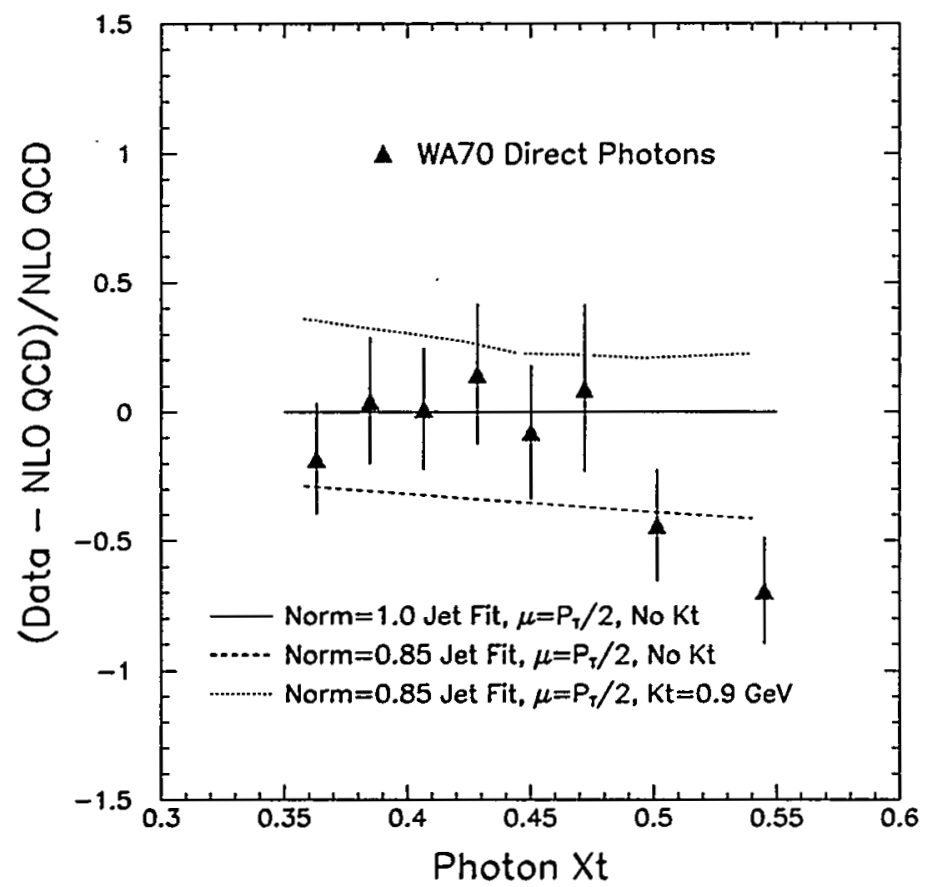

Figure 3: The hypothesis of additional gluons at high $x$ does not spoil the NLO QCD agreement with WA70 photon data for reasonable assumptions about $\mu$, normalization and $k_{r}$. 


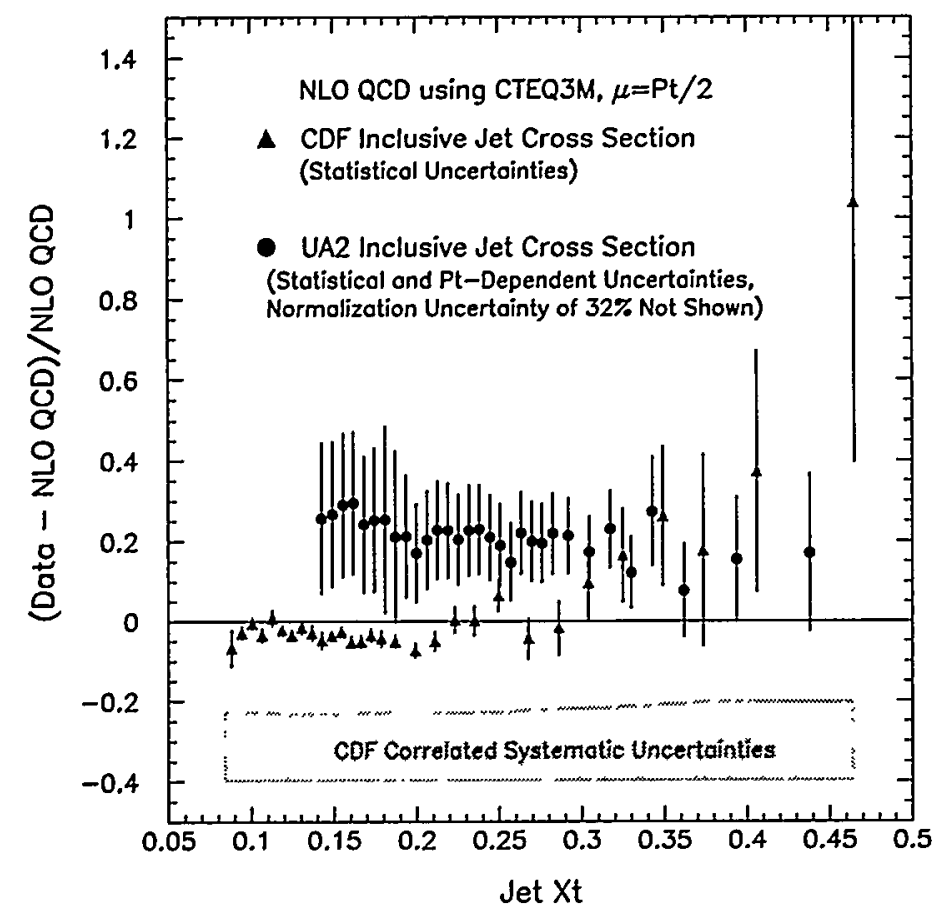

Figure 4: The nominal structure functions describe the shape of the UA2 jet spectrum which does not show the trend of the CDF jet spectrum.

\section{Direct Photons}

Steve Kuhlmann, Bob Blair and Dan Crane have been working on analyses involving photons identified in the central calorimeter. Particular emphasis has been on the study of production of photons with charm. Electrons, muons and $D^{*}$ s have been used to signal the presence of charm with photons and the results are similar, Fig. 5. The observed cross section is somewhat higher than the leading order expectation, which may be due to higher order effects or the charm structure function. 


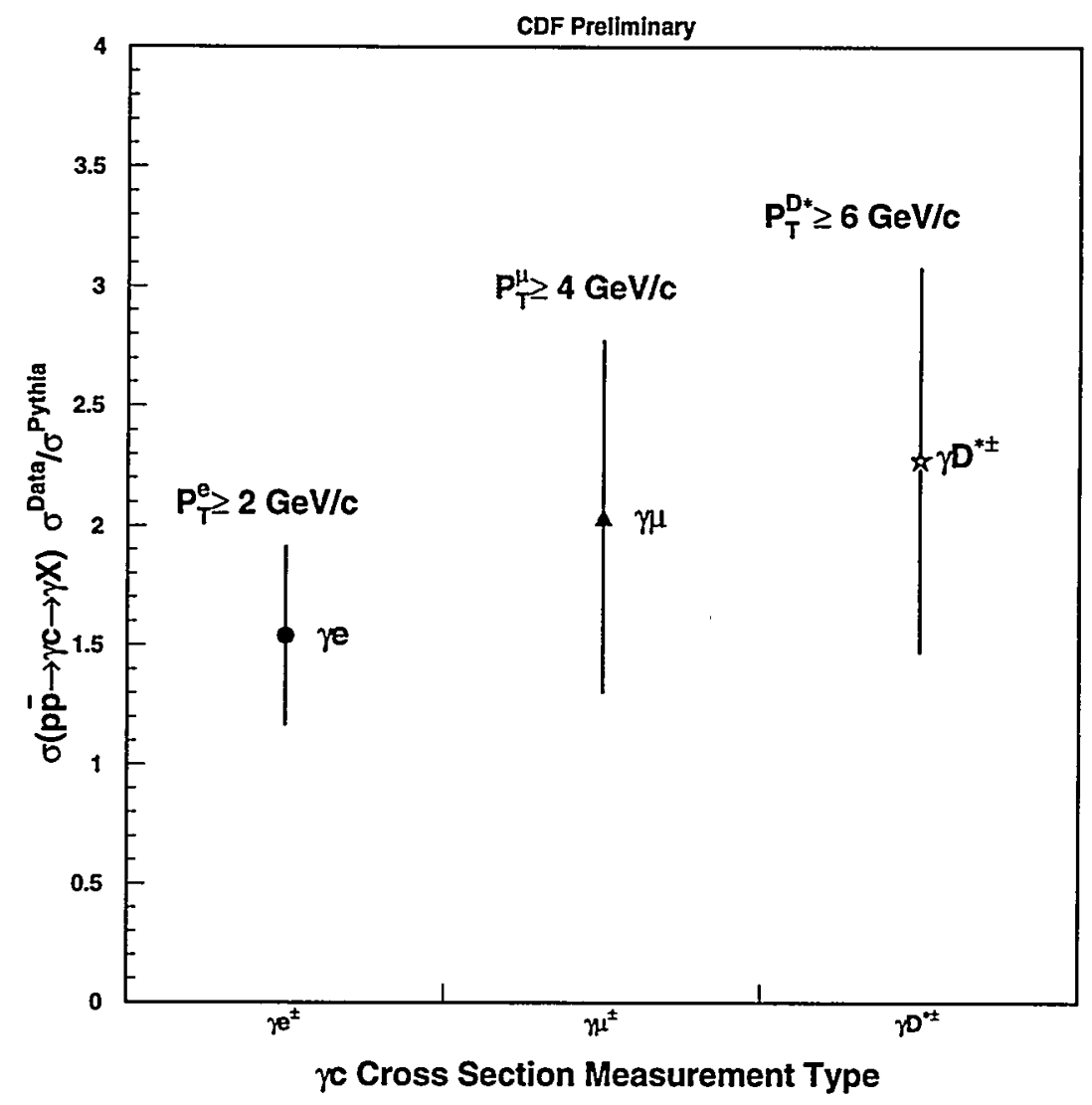

Figure 5: The rate of photon charm production observed/expected for three charm signatures.

\section{b Physics}

Karen Byrum and Barry Wicklund are continuing their work on measuring the $\mathrm{b}$ cross section using inclusive electrons. The full Run $1 \mathrm{~b}$ inclusive electron dataset is now available on disk at Argonne. Barry has continued working with his former student Fumi Ukegawa, now at Pennsylvania, on obtaining lifetimes for the neutral and charged $\mathrm{B}$ mesons using semileptonic decays. The charged versus neutral B mesons have a strong correlation to charged versus neutral D or D* mesons. The one neutral and three charged charm signals are shown in Fig. 6. The associated transverse vertex distributions allow lifetimes of $1.56 \pm 0.13 \pm 0.06$ and $1.54 \pm 0.08 \pm 0.06$ ps charged and neutral B mesons to be extracted, with the ration $1.01 \pm 0.11 \pm 0.02$. This measurement is nearly as accurate as the $\mathrm{CDF}$ reconstructed exclusive measurement. 

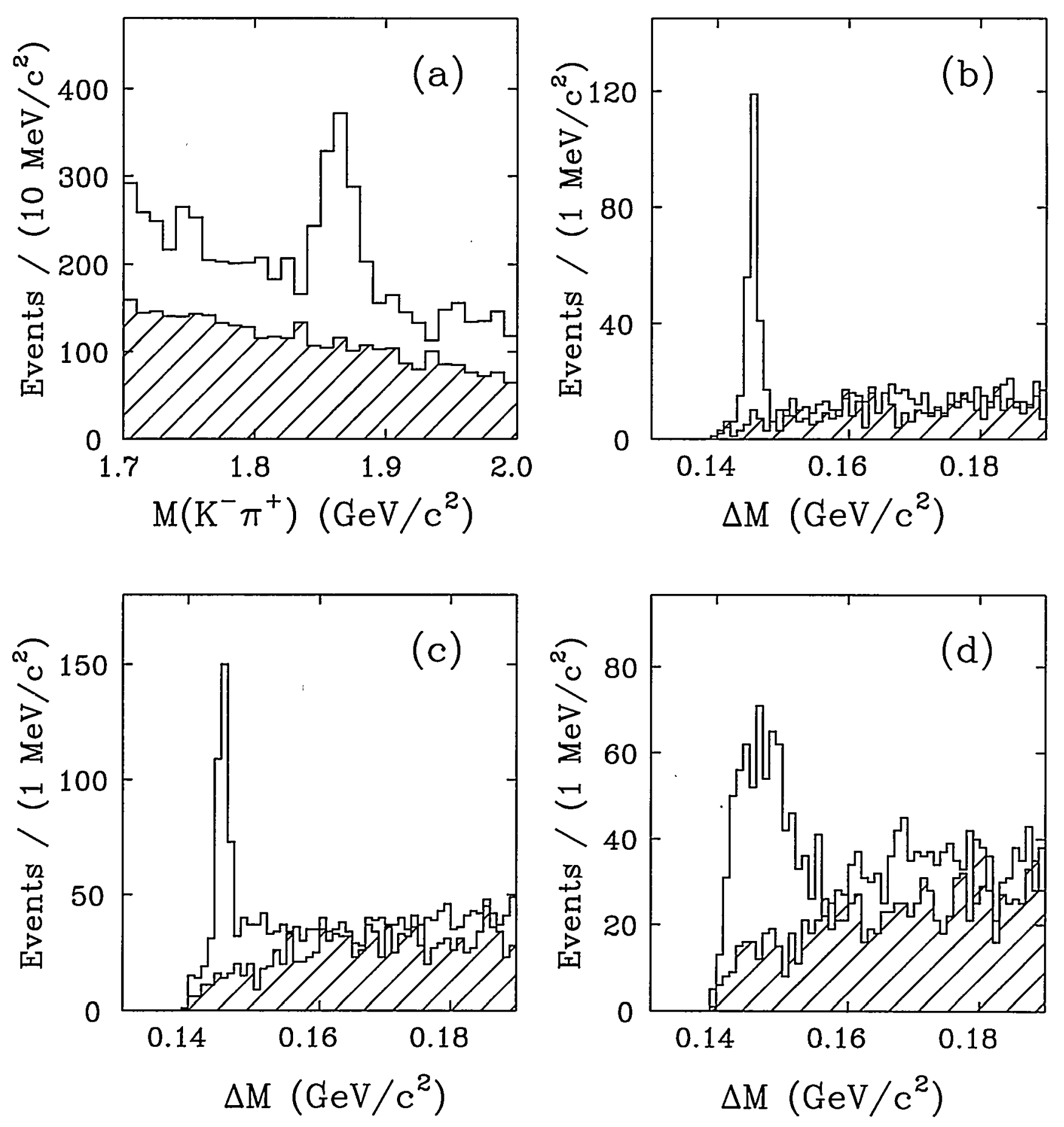

Figure 6: For the neutral charm the $D^{0} \rightarrow K \pi$ signal (a) is antiselected for $D^{*}$. For charged charm the $D^{*}$ mass difference is shown for $D^{*^{*}} \rightarrow \pi^{ \pm} D^{0}$ where the $D^{0}$ decays to, with charge conjugate, (b) $K \pi^{+}$, (c) $K \pi^{+} \pi^{+} \pi$ and (d) $K \pi^{0} \pi^{+}$. The shaded area is for wrong sign combinations, $\mathrm{x} 0.5$ for clarity in (a). 


\section{b. CDF Summary of Active Data Taking}

Running resumed after an extended summer shutdown. Given the small additional dataset likely to be obtained, the b-physics group was allowed to install various test triggers. Both luminosity and detector performance were disappointing in running at $1800 \mathrm{GeV}$ but in the last three weeks of running before the Christmas shutdown a very good sample of 0.5 $\mathrm{pb}^{-1}$ at $630 \mathrm{GeV}$ was obtained and the program of diffractive studies using a Roman pot spectrometer and microplug calorimeters became operational.

Marcus Hohlmann and Larry Nodulman have continued supporting the monitoring of data quality. Steve Kuhlmann, Tom LeCompte and Larry Nodulman continued cycles of being shift leaders, and Bob Wagner took an occasional week.

Running ended in February with a disappointing additional data sample. The detector will be removed from the collision hall after a period of machine studies and a source calibration for the central EM calorimeter

\section{c. CDF Planned Activities and Planning Issues}

The current expectation for run 2 is to roll the detector in during 1999. Unfortunately, the funding profiles given to both $\mathrm{CDF}$ and D0 seem more appropriate to completing the upgrades in 2000, and seem optimistic in expecting a substantial increase after two years, making further delay seem likely. The main injector program, on the other hand, seems likely to be able to provide luminosity on schedule. Both collaborations and Fermilab are facing difficult decision on lowering the scope of upgrades, staging, and/or delay.

The PAC and the laboratory have redefined Run 2 to start at a luminosity of $10^{32} \mathrm{~cm}^{-2} \mathrm{sec}^{-1}$ and grow by a factor 2 or more from there, although a weaker start and slower growth may result from funding limitations. A permanent magnet antiproton recycler ring in the main injector tunnel is included in the main injector project.

The Argonne group, with Bob Wagner in the lead, has worked with the Duke and Michigan groups and others to develop a proposal to the CDF collaboration ${ }^{(1)}$ for a straw tracker based on SDC experience to replace the CTC. An alternative being considered, is a smaller open cell chamber like the CTC which would give have lower mass and thus less multiple scattering. The straw system has more material but would have better performance at higher luminosity beyond the nominal $2 \times 10^{32} \mathrm{~cm}^{-2} \mathrm{sec}^{-1}$.

In September the collaboration had a technical review and godparent review of the two alternatives. The technical review found both alternatives viable and the godparent review favored the straw proposal, and this was reported at length to the $\mathrm{PAC}^{(2)}$. 
Cost and schedule concerns have caused the competition to be reopened, with a cost and schedule review within the collaboration to be complete in early March. A comprehensive TDR is planned for the overall upgrade.

We have reached agreement in detail on our participation in the program of work on front end electronics. Karen Byrum has taken over this project from Marcus Hohlmann who has moved on to worry about his thesis analysis.

The high luminosity physics report organized by Dan Amidei and Chip Brock called TeV2000 ${ }^{(3)}$ included among other things a study of $\mathrm{H} \rightarrow b b$ prospects by Steve Kuhlmann. Fermilab has now taken over this effort, renamed 'TeV33'. Several members of our group are expected to participate in this summer's Snowmass planning exercise.

\section{References}

(1) D. Amidei et al., CDF-3350.

(2) F. Abe et al. (CDF), CDF-3459.

(3) Report of the TeV2000 Study Group, D. Amidei and C. Brock eds., the latest draft can be found at http://www/pa.msu.edu/ ftp/pub/tev 2000/writeup/.

\section{(L. Nodulman)}

\section{A. 4 Non-Accelerator Physics at Soudan}

\section{a. Physics results}

During the last half of 1995 Soudan physicists continued to focus their analysis effort on the identification and characterization of atmospheric neutrino interaction events in Soudan 2 data. The initial goal of this analysis is to measure the $v_{u} / v_{\mathrm{e}}$ "flavor" ratio for atmospheric neutrinos using quasi-elastic events in Soudan 2. Differences between measured ratios and those predicted by Monte Carlo simulations have been interpreted as evidence for neutrino oscillations.

About 2.7 fiducial kiloton years of atmospheric neutrino data have been recorded by Soudan 2 since operation began in 1988. The initial two kton-year data set has been divided into four half-kiloton year samples for analysis purposes. A preliminary analysis of the first two samples was completed in 1993, using an early version of the Monte Carlo simulation. An extensive upgrade of the Monte Carlo simulation software was completed in early 1995, providing more accurate representations of neutrino interactions and of the detector response to particles produced in these events. At the same time, a new analysis procedure was adopted to provide a better understanding of systematic errors in the atmospheric neutrino flavor ratio measurement. 
The new analysis procedure mixes a large sample of Monte Carlo neutrino interaction events with actual contained event data. Monte Carlo events are processed by the same software as Soudan 2 data events, and are scanned by physicists along with actual data. The physicist classification of contained events is an inherently subjective process involving pattern recognition skills and physics judgments. In the new procedure, the physicist scan is carried out by two independent teams on a mixed, unlabeled sample of Monte Carlo and data events. Comparison of event classifications made by the two analysis teams gives a measure of the systematic uncertainties of the scanning process. Comparison of the classification of Monte Carlo events with the actual event types measures the event classification confusion matrix, and allows physics results to be corrected for misidentification effects.

The two analysis teams met for the first time to compare their results for the third half-kiloton year data sample in July. Despite the fact that two groups used somewhat different scan rules, the $\nu_{\mu} / v_{\mathrm{e}}$ ratio obtained from their samples of quasi-elastic neutrino events were nearly the same, and were also similar to the ratio obtained in 1993 for the first 1.0 kton year data set. The blind scanning of Monte Carlo events produced a number of important insights into event misidentification by scanners. At the end of 1995, the details of the scanning procedure and corrections for systematic effects were still being refined, and the scanning of Monte Carlo events for the first two data samples (the first kiloton year exposure) had begun. The collaboration plans to publish its ratio measurement based on a 1.5 kton year exposure during 1996.

Atmospheric neutrino events and the search for nucleon decay in Soudan 2 were studied by William Leeson in his Tufts University Ph.D. thesis, which was completed in December. One particularly important result is an experimental evaluation of the background from neutron and gamma interactions (produced by muons interacting in the rock surrounding the experiment) in the sample of atmospheric neutrino event candidates. For the 30 single-track candidate neutrino events in the first $1.0 \mathrm{kton}$ year sample, he deduced the neutron/gamma background to be $5.7 \pm 3.5$ events; a background of $2.0 \pm 3.7$ was found for the 35 single-shower events.

The Soudan 2 experiment has been able to perform this background study, which is unique among underground neutrino and nucleon decay experiments, because it has accumulated a large sample of contained "rock events" (from neutrons and gammas emerging from the cavern walls) which were tagged by the presence of hits in the active shield counters. Figures 1 and 2 show the penetration depth distributions for the tagged rock events for showers and tracks, respectively, while Figures 3 and 4 show the same distributions for the neutrino event candidates (which do not produce hits in the active shield). Each neutrino distribution was fitted to a sum of the shapes obtained from the rock events and the Monte Carlo neutrino events. The resulting background levels (given in the preceding paragraph) do not substantially change the value for the atmospheric neutrino flavor ratio obtained from Soudan 2 data. 
Three new Soudan 2 limits on nucleon decay are calculated in Leeson's thesis, which uses the first $1.0 \mathrm{kton}$ year data sample $(\tau / \mathrm{B}$ is the lifetime divided by the branching ratio):

Decay mode $\quad \tau B$ 90\% CL limit $\quad$ Bkgnd (subtracted) Expected bkgnd

$\begin{array}{llll}n \rightarrow \mathrm{e}^{+} \mathrm{e}^{-} & 5.0 \times 10^{31} \text { years } & 1 \text { event } & <0.1 \text { event } \\ n \rightarrow \bar{v} \eta & 1.6 \times 10^{31} \text { years } & 1 \text { event } & <0.1 \text { event } \\ n \rightarrow \bar{v} \pi^{\circ} & 3.2 \times 10^{31} \text { years } & 1 \text { event } & <0.9 \text { event }\end{array}$

While these limits are not yet as restrictive than those obtained by the Kamiokande experiment, the lower background levels in our experiment will give Soudan 2 an excellent sensitivity when our full exposure is analyzed. Other thesis students at Minnesota, Oxford, and Tufts are also searching for evidence of nucleon decay in a number of additional modes.

Finally, Leeson's thesis examines the hypothesis that the lower than expected ratio of muon to electron neutrino interactions may be due to an excess of single shower events from proton decay, $\mathrm{p} \rightarrow \mathrm{e}^{+} v v$, instead of the more conventional explanation in terms of muon neutrino oscillations. It is difficult to distinguish between the two hypotheses because the absolute rates of neutrino interactions cannot be predicted as accurately as the ratio of muon to electron events. However, the ability of Soudan 2 to detect recoil proton tracks allows neutrino interactions to be distinguished from proton decay events in a way which is not accessible to water Cerenkov detectors. About a third of the quasi-elastic neutrino interactions, for both electron and muon events, are expected to have visible recoil proton tracks, whereas nucleon decay events will not have such recoil tracks. The data show that 6 out of 35 single-shower events $(17 \pm 7 \%)$ and 9 out of 30 single-track events $(30 \pm 10 \%)$ have visible recoil proton tracks. The difference is compatible with the proton decay hypothesis, but is not statistically significant in this initial 1 kton year sample.

Soudan 2 data are also being used for a number of ongoing cosmic-ray physics analyses, which will be the subjects of future Ph.D. theses and publications. These include the study of primary cosmic ray composition using multiple muon events (described in the previous Semiannual Report) and the use of underground muon tracks to search for astrophysical point sources. A search for very energetic $(100 \mathrm{TeV})$, nearly horizontal muons is also under way. Such muons would result from very high energy. neutrino interactions in the rock surrounding the detector, and would be identified by the presence of a catastrophic interaction (depositing $>40 \mathrm{GeV}$ ) along the track, which is characteristic of very high energy muons. Some models predict that active galactic nuclei will produce a flux of such neutrinos which would be detectable in Soudan 2. Current work includes a search for candidate events, a study of detection efficiency, and an evaluation of possible backgrounds. 


\section{b. Experimental apparatus improvement}

The Soudan 2 calorimeter-module upgrade project was completed in November when the last rebuilt halfwall (an array of eight modules which is read out as a single unit) came into operation. During the last half of 1995 , seven halfwalls and twelve calorimeter modules were rebuilt. Over the two-year project, 80 of the worst-performing modules (out of 224 total) were removed from the detector to improve wireplane gain uniformity and to repair leaks. In addition, anode high voltage splitter hardware was installed on all remaining halfwalls so that each individual wireplane may be operated at its own optimum high voltage. The full 963-ton Soudan 2 detector has been operating continuously since November.

Three of the four panels of the new Oxford "crack filler" array began operation during the last half of 1995. The first two panels were installed, but not turned on, earlier in the year; 114 of the total 152 proportional tubes in the array are now in operation. Tubes for the fourth panel are being prepared for installation during 1996. This array of proportional tubes is being installed immediately above the upper layer of Soudan 2 calorimeter modules and is constructed of tubes originally used in the Tasso detector at DESY. It will provide enhanced detection of cosmic ray muons which might otherwise enter the calorimeter undetected through cracks between modules and halfwalls to produce fake contained events. Such detection is already provided by the cavern-liner active shield which has been in operation for many years. While the original shield is still thought to provide adequate coverage, crack-penetrating muons are potentially such a serious background for neutrino and nucleon decay events that the redundancy provided by the new crack filler array is important, if only to confirm the effectiveness of the original shield.

Argonne physicists continued to make substantial contributions to the maintenance and operation of the detector. Major activities included ongoing improvements of detector and electronics performance, and coordination of the module upgrade project. Argonne physicists also continued the development of software to make use of $\mathrm{dE} / \mathrm{dx}$ information from the detector.

\section{c. Summary of active data acquisition}

The Soudan 2 detector is operated continuously for physics data whenever other activities permit. In practice, most data are recorded during night and weekend periods when installation or maintenance work is not in progress, and the underground laboratory is unoccupied. The anode-cathode edge trigger, which was devised for neutrino interactions and nucleon decay, has high efficiency for cosmic-ray muon tracks as well. All data are processed at Soudan by track reconstruction programs, and the analysis results are recorded on $8 \mathrm{~mm}$ magnetic tape cassettes for distribution to the collaborating institutions. 
The Soudan 2 experiment continued routine data acquisition for contained events (neutrinos and nucleon decay) and cosmic ray muons during the second half of 1995. In addition, data from the $40 \mathrm{~m}^{2}$ surface array were recorded in coincidence with Soudan 2 in order to measure the energies of some of the cosmic ray air showers which produce underground muon events. Data from a wide-angle air Cerenkov air-shower detector were also recorded in coincidence with Soudan 2 on clear moonless nights.

The Soudan 2 detector itself recorded data for 131 days of livetime during the sixmonth period, giving a duty cycle of $72 \%$. This brought the total Soudan 2 exposure to 5.3 years, providing a total exposure of 2.7 fiducial kiloton years useful for nucleon decay and atmospheric neutrino physics.

\section{d. Planning activities}

During 1996, the last panel of the Oxford crack filler array will be brought into operation. The active shield ceiling will be further enhanced by the installation of the last two "HPW" ceiling panels. Six of the eight panels of this array are already in operation. The HPW ceiling array uses proportional tubes from the HPW nucleon decay experiment to increase the detection efficiency for cosmic ray muons entering the Soudan 2 detector in the cracks between modules. The HPW tubes are oriented perpendicular to the original Tufts shield tubes in order to provide additional position information for cosmic ray muons. The two dimensional muon information is particularly useful for the reconstruction of multiple muon events in the active shield.

Soudan 2 experimenters also devoted a major effort to planning for the new MINOS long baseline neutrino oscillation experiment, which is described elsewhere in this report. In September the MINOS experiment received a strong endorsement from the HEPAP Subpanel on Neutrino Oscillation Experiments. Argonne physicists played major roles in the planning for MINOS and in the presentation of the experiment to the HEPAP subpanel and the Fermilab PAC.

(D. Ayres) 


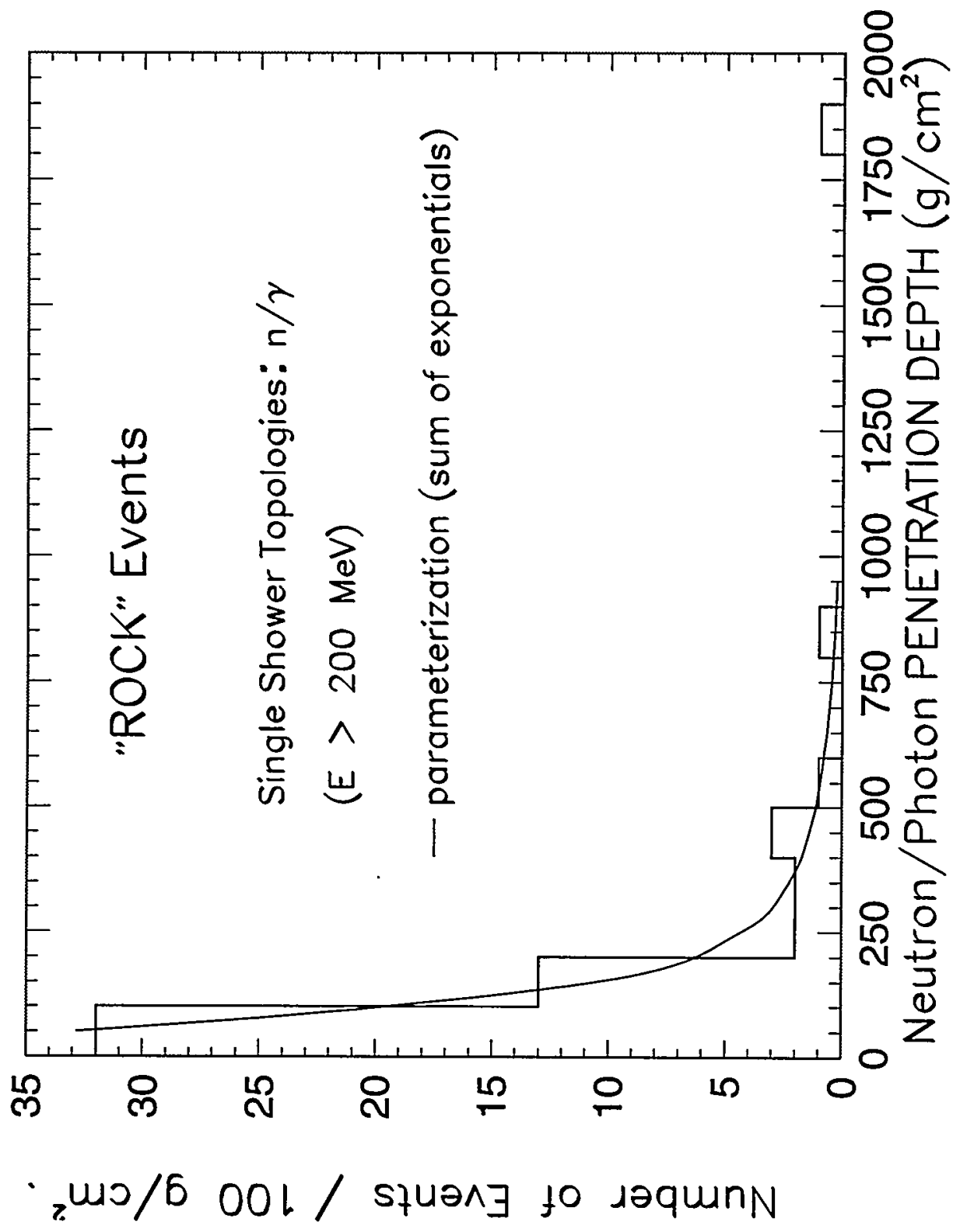

Fig. 1. Penetration-depth distribution for neutron- and gamma-induced single shower events with shower energies greater than $200 \mathrm{MeV}$, from contained Soudan 2 events which were flagged as background ("rock" events) by the active shield. The curve is a two-exponential parametrization of the data. 


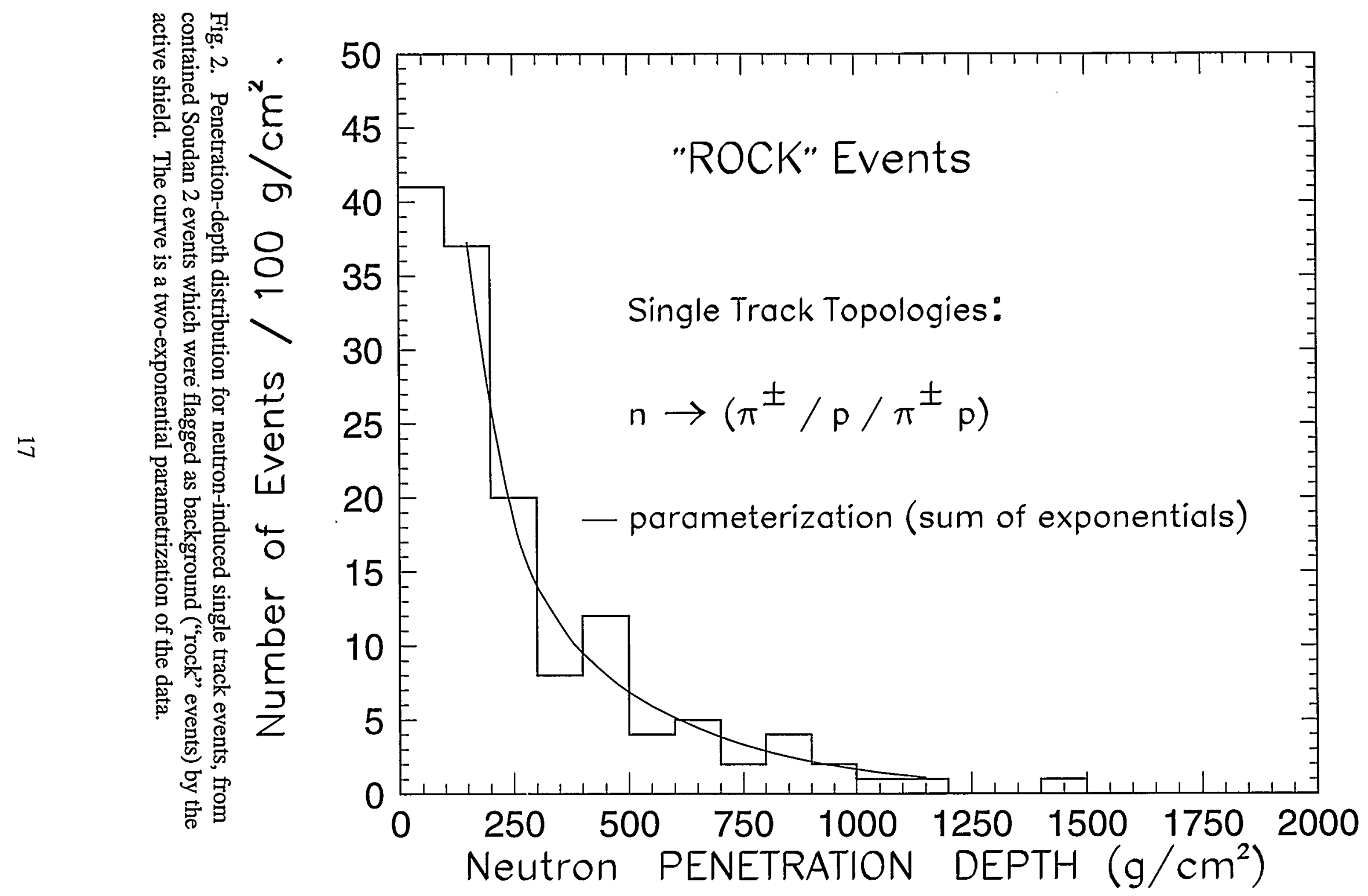




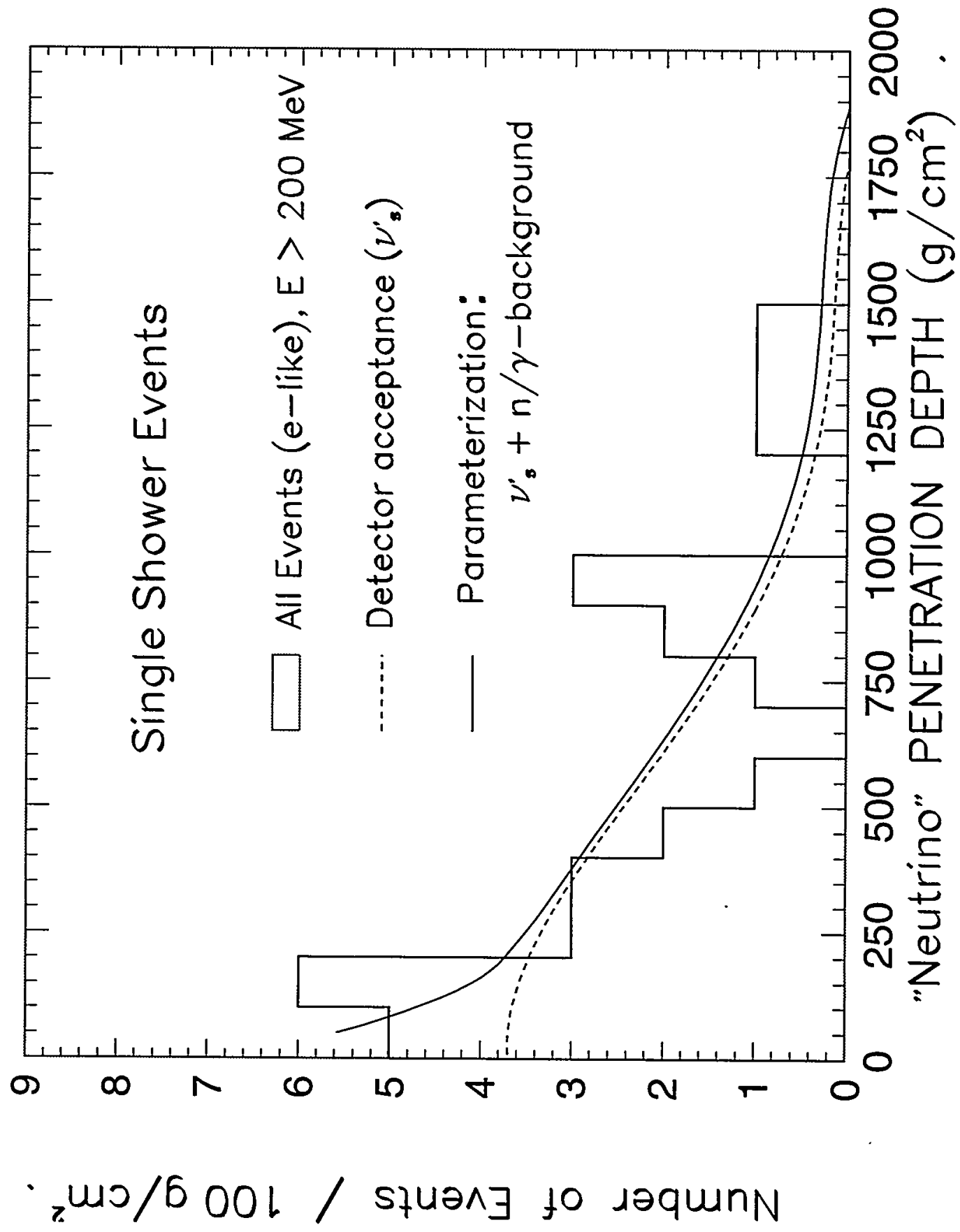

Fig. 3. Penetration-depth distribution for contained single-shower neutrino event candidates with shower energies greater than $200 \mathrm{MeV}$, from Soudan 2 events with no active shield hits. The dashed curve shows the detector acceptance and the solid curve is a fit to the sum of the shapes obtained from rock events and Monte Carlo neutrino events. 


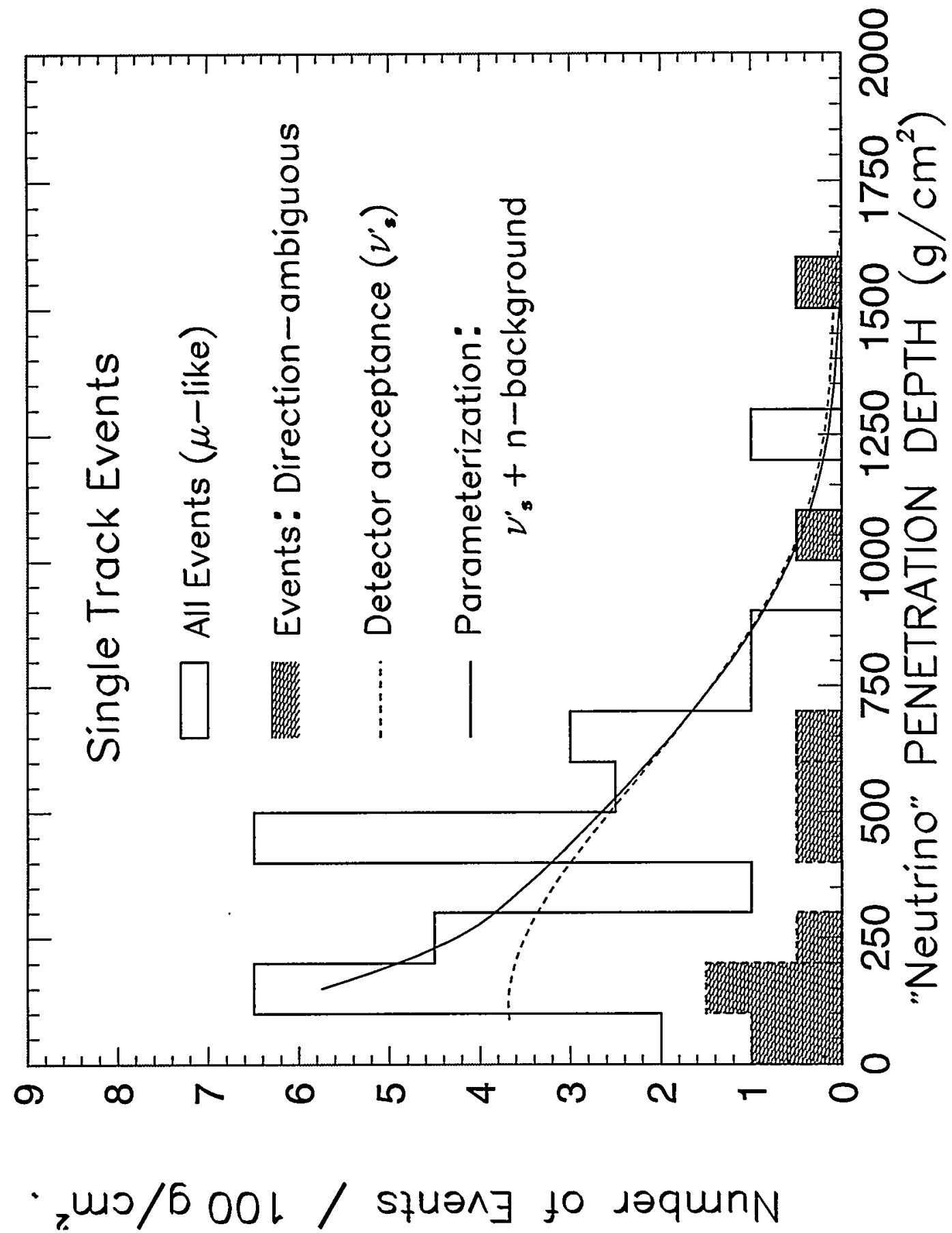

Fig. 4. Penetration-depth distribution for contained single-track neutrino event candidates, from Soudan 2 events with no active shield hits. The dashed curve shows the detector acceptance and the solid curve is a fit to the sum of the shapes obtained from rock events and Monte Carlo neutrino events. 


\section{A. 5 ZEUS Detector at HERA}

\section{a. Physics Results}

Seven papers were published in this period and several more manuscripts were submitted for publication.

Because of the asymmetric beam energies in HERA, the hadrons from the fragmentation of the photon remnant in resolved photoproduction can be measured in the main ZEUS detector. A sample of events with two high $P_{t}$ jets $\left(P_{t}>6 \mathrm{GeV}, \eta<1.6\right)$ and a third cluster in the direction of the incident photon were isolated using a clustering algorithm. The cm energy spans the range $130<\mathrm{W}<270 \mathrm{GeV}$. The third cluster has a mean transverse momentum with respect to the jet axis of $2.1 \pm 0.2 \mathrm{GeV}$, substantially larger than the expectation of the PYTHIA Monte Carlo simulation. After correcting the Monte Carlo simulation for this effect, the mean transverse energy per particle as a function of the jet energy E3 as well as the longitudinal and transverse energies are well reproduced as shown in Figs. 1a and 1b. The energy flow around the jet axis is shown in Fig. 1c. Figs. 1d, 1e and 1f compare the properties of the remnant jet with those of the two high $\mathrm{P}_{\mathrm{t}}$ jets. In doing this comparison, the high $\mathrm{P}_{\mathrm{t}}$ jets were selected to have energies between 8 and $15 \mathrm{GeV}$, comparable to those of the remnant jet.

The first study of high $Q^{2}$ neutral and charged current scattering was completed and the cross sections measured. As seen in Fig. 2, the cross sections become equal at $Q^{2}$ values comparable to the masses of the $\mathrm{Z}$ and $\mathrm{W}$ bosons. The neutral current cross section falls by six orders of magnitude from the lowest $Q^{2}$ value of $10 \mathrm{GeV}^{2}$ up to those shown in the figure. The data agree well with the expectations of the standard model.

Further information on diffractive processes in both DIS and photoproduction became available in this period. Measurements of the diffractive DIS cross section over a range of $Q^{2}$ and $x_{\mathrm{bj}}$ show that the diffractive fraction is independent of these variables at about the $10 \%$ level as shown in Fig. 3. If the process is mediated by pomeron exchange, then the cross section should factorise:

$$
F_{2}{ }^{D(4)}\left(\beta, Q^{2}, x_{I P}, t\right)=f_{I P}\left(x_{I P}, t\right) \cdot F_{2}{ }^{p}\left(\beta, Q^{2}\right)
$$

where $\mathrm{F} \begin{aligned} & D(4) \\ & 2\end{aligned}$ is defined by:

$$
\frac{d^{4} \sigma_{d i f f}}{d \beta d Q^{2} d x_{I P} d t}=\frac{2 \pi \alpha^{2}}{\beta Q^{4}}\left[\left(1+(1-y)^{2}\right) F_{2}^{D(4)}-y^{2} F_{L}^{D(4)}\right]\left(1+\delta_{z}\right)\left(1+\delta_{r}\right)
$$

The results shown are calculated neglecting the longitudinal structure function $F_{L}$, the $Z$ exchange contribution $\delta_{\mathrm{z}}$ and the radiative corrections $\delta_{\mathrm{r}}$. After integrating over $t$, the unmeasured momentum transfer at the proton vertex, the results for $F_{2}{ }^{D(3)}$ are shown in Fig. 4 as a function of $\mathrm{x}_{\mathrm{IP}}$ for different selections of $\mathrm{Q}^{2}$ and $\beta$. The latter is the fraction of the pomeron momentum that enters into the hard scattering. The data fall rapidly with 
$x_{I P}$. The line is a fit to $b\left(1 / x_{\mathrm{IP}}\right)^{a}$ with a fixed value of the exponent $a$. The result is $a=$ $1.30 \pm 0.08{ }_{-0.14}^{+0.08}$ This value corresponds to a pomeron intercept of $\alpha_{I P}(0)=1.15$ somewhat larger than the standard value of 1.08 derived by Donnachie and Landshoff from hadron scattering data, but below the value of 1.5 predicted by Lipatov. The $\beta$ and $Q^{2}$ dependences are shown in Fig.5. The $Q^{2}$ dependence is flat but the $\beta$ dependence is somewhat softer than the hard $\beta(1-\beta)$ form shown by the dashed lines.

Further insight into the pomeron structure is given by a comparison of these results with hard diffractive photoproduction. Hard scattering is selected by requiring a high $E_{\mathrm{t}}$ jet in the event. Fig. 6 shows the measured cross section as a function of pseudorapidity, $\eta$, compared to three assumptions about the pomeron structure. The hard gluon assumption is the only simple model to fit the data. A comparison with the DIS data is shown in Fig. 7. The two bands cross near $C_{g}=0.6$, where $C_{g}$ is the fraction of the pomeron momentum carried by gluons. The data are compatible with $\Sigma_{I P}=1$, which is the momentum sum rule. Since the pomeron is not a particle in the usual sense, the momentum sum rule may or may not be satisfied. These results show that hard gluons are an important constituent of the pomeron.

Further information comes from the measurement of exclusive $\rho^{o}$ production in DIS. The results are shown in Fig. 8. The mass spectrum of Fig. 8a exhibits a clear $\rho^{o}$ peak with little background. The cross sections are higher than those reported by the NMC collaboration at lower energies as seen in Fig. $8 \mathrm{~b}$. The decay angular distribution of the $\rho^{o}$ (Fig. 8c) indicates dominance by longitudinal photons in agreement with earlier data as seen in Fig. 8d. The cross sections are compared to lower energy and lower $Q^{2}$ data in Fig. 9. The cross sections for photoproduction $\left(Q^{2}=0\right)$ rise only slowly with energy as expected from soft pomeron models whereas at higher $Q^{2}$ the cross section rise is much faster. Such a behavior is at variance with the soft pomeron model of Donnachie and Landshoff but agrees with the calculations of Brodsky and collaborators. In this model the pomeron is considered to be a two gluon state so the cross section is proportional to the square of the gluon density in the proton.

A first determination of the strong coupling constant, $a_{\mathrm{s}}$ was made from a measurement of the two jet cross sections. The jets were identified using the JADE clustering algorithm. A cut on the angular distribution of parton emission in $\gamma^{\prime}$-parton $\mathrm{cm}$ system minimizes theoretical and experimental uncertainties arising from colinear partons. The resulting values of $\alpha_{s}$ in three $Q$ intervals is shown in Fig. 10. When extrapolated to $Q=M_{Z}$ the values yield $\alpha_{s}=0.117 \pm 0.005{ }_{-0.005}^{+0.004} \pm .007$ where the last error reflects the scale uncertainty in the theory.

A paper giving the first results on $\mathrm{K}^{0}$ multiplicities was also published in this period. 


\section{b. HERA and ZEUS Operations}

The $1995 \mathrm{e}^{+} \mathrm{p}$ data taking was completed at the end of November with HERA delivering $12.2 p b^{-1}$ and Zeus logging 7.1 $p b^{-1}, 3.6 p b^{-1}$ of which were taken with the Leading Proton Spectrometer. The HERA delivered luminosities for the four operating periods, summarized in Fig. 11, showing a steady improvement from year to year although the delivered luminosity for 1995 fell below the $15 \mathrm{pb}^{-1}$ expected. This is ascribed to some inefficiencies in the operation connected with an unusual number of power failures. The mean peak luminosity was $4 \times 10^{30} \mathrm{~cm}^{-2} \mathrm{sec}^{-1}$, and the average data run delivered $51 \mathrm{pb}^{-1}$ in 6.2 hours. The overall efficiency was $35 \%$. A few weeks of data were taken with shifted ep vertices to extend the kinematic coverage of the detector.

A number of significant improvements are being made to the HERA hardware during the 1995-1996 shutdown, with the aim of delivering $15 \mathrm{pb}^{-1}$ during the reduced data taking period from July through November 1996. The proton ring in the West area has been extensively modified to accommodate the HERA-b experiment. Beam tests done in 1995 show that it is possible for the HERA-b target to be in place without giving excessive backgrounds in ZEUS, although the situation is not completely understood.

Plans are being made for a test run with reduced proton energy $(450 \mathrm{GeV})$ during 1996. Such data will be needed to measure the longitudinal structure function. Operations with electrons is expected to resume in 1997.

(M. Derrick) 

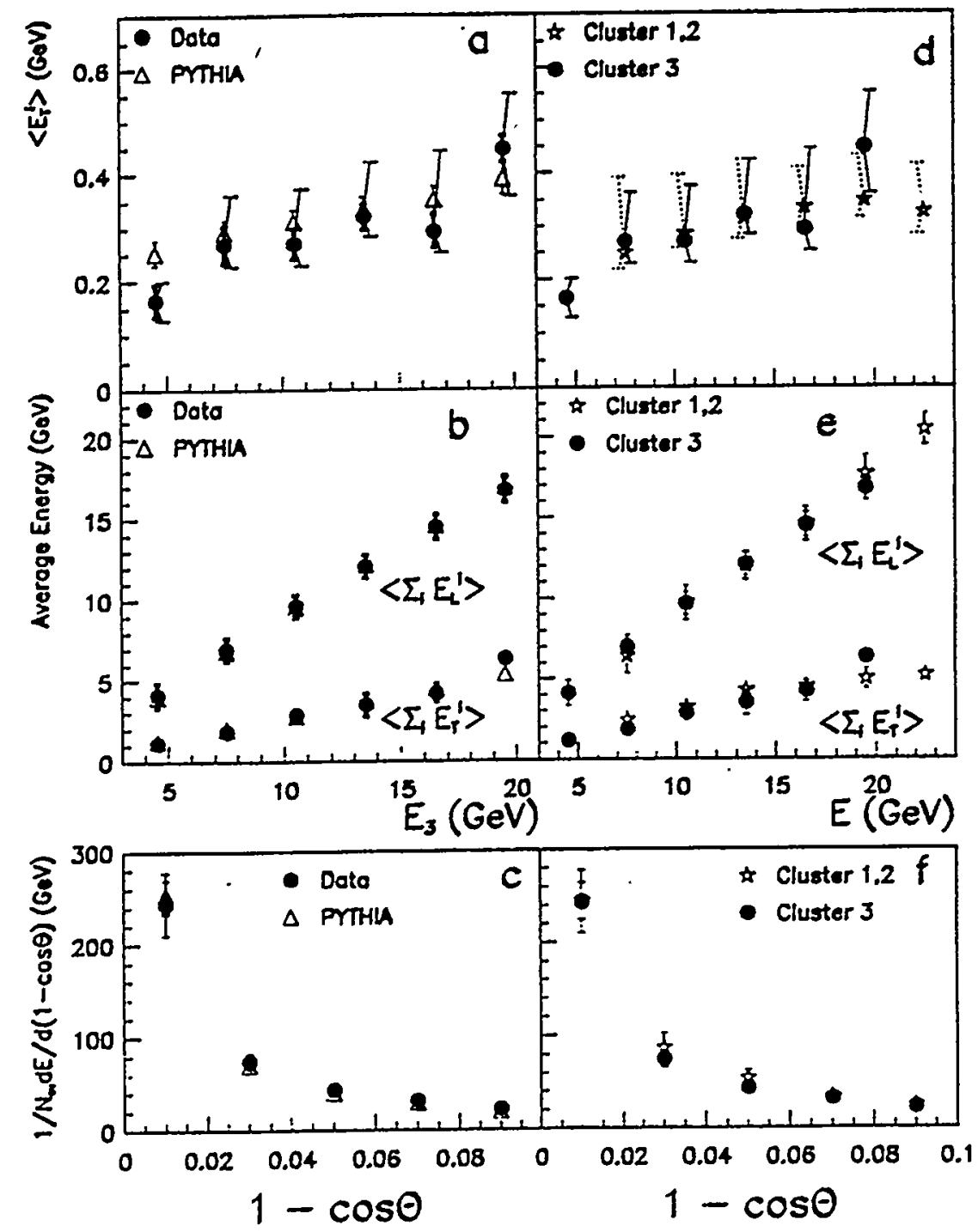

Fig. 1. (a-c) Comparison between the data and the hadron level Monte Carlo expectations. (a) The mean value of $\left(E_{T}^{1}\right)$, the average energy transverse to the cluster axis per particle, as a function of the cluster energy. (b) The average values of the total transverse $\left(\Sigma_{1} E_{T}^{1}\right)$ and total longitudinal $\left(\Sigma_{1} E_{L}^{1}\right)$ energy. (c) The flow of energy around the cluster axis. (d-f) Comparison between the photon remnant (cluster 3) and the two high - $\mathrm{pT}$ jets. The error bars show the systemmatic and statistical errors added in quadrature. 


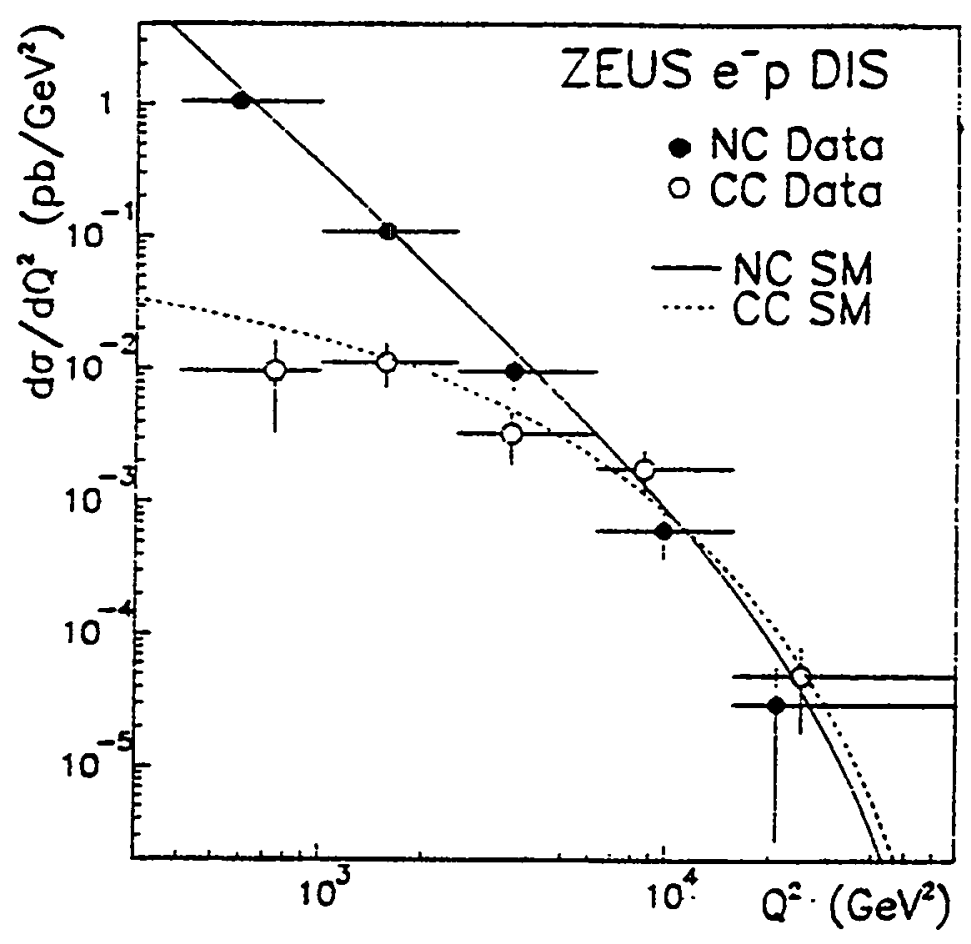

Fig. 2. $d \sigma / d Q^{2}$ for CC and NC DIS. The points with errors are the data, and the curves are the standard model Born cross sections. The data are plotted at the average $Q^{2}$ of the events in each bin. 


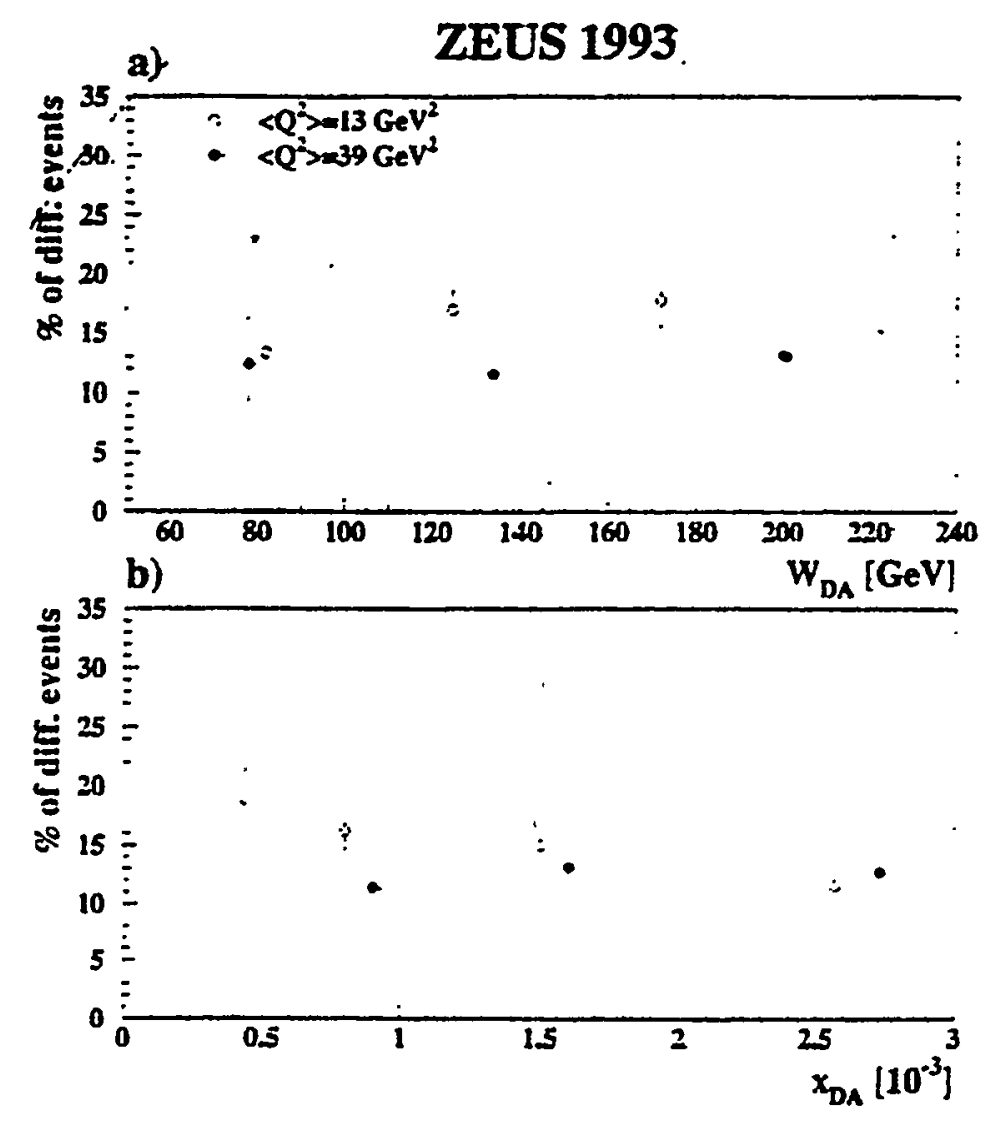

Fig. 3. Observed fraction of diffractive events as a function of $W_{D A}$ and $\chi_{D A}$ in two $Q^{2}$ intervals. The data are fitted to $\mathrm{NZ}$ model for diffractive processes and the CDMBGF model for the non-diffractive contribution. The errors are the statistical errors combined in quadrature with $50 \%$ of the non-diffractive DIS background. 


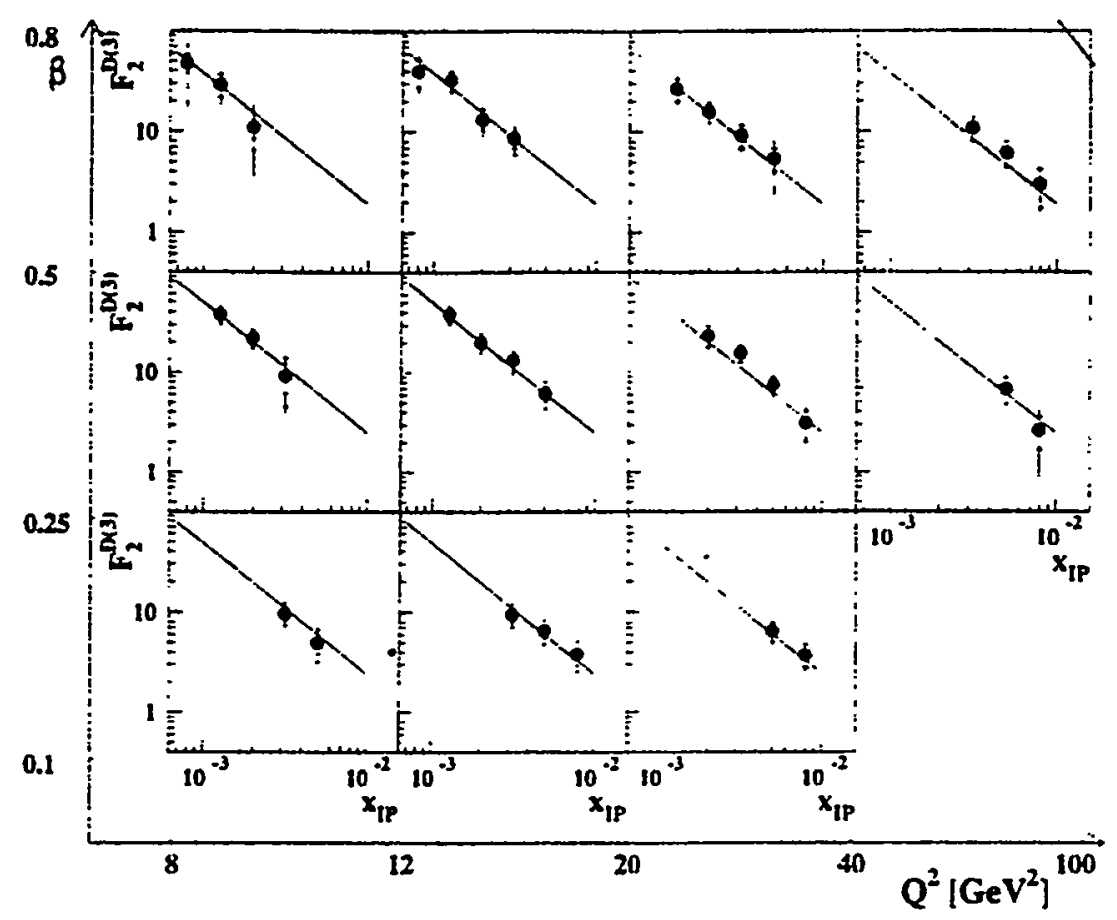

Fig. 4. The results of $F_{2}^{D(3)}\left(\beta, Q^{2}, \chi_{I P}\right)$ compared to the parametrisation ' discussed in the text. The inner error bars show the statistical errors, the outer bars correspond to the statistical and DIS event selection systematic errors added in quadrature, and the full line corresponds to the statistical and total systematic errors added in quadrature. Note that the data include an estimated $15 \%$ contribution due to double dissociation. The overall normalization uncertainty of $3.5 \%$ due to the luminosity uncertainty is not included. The outer axes indicate the range of $\beta$ and $Q^{2}$ where the measurements have been performed. 


\section{ZEUS 1993}
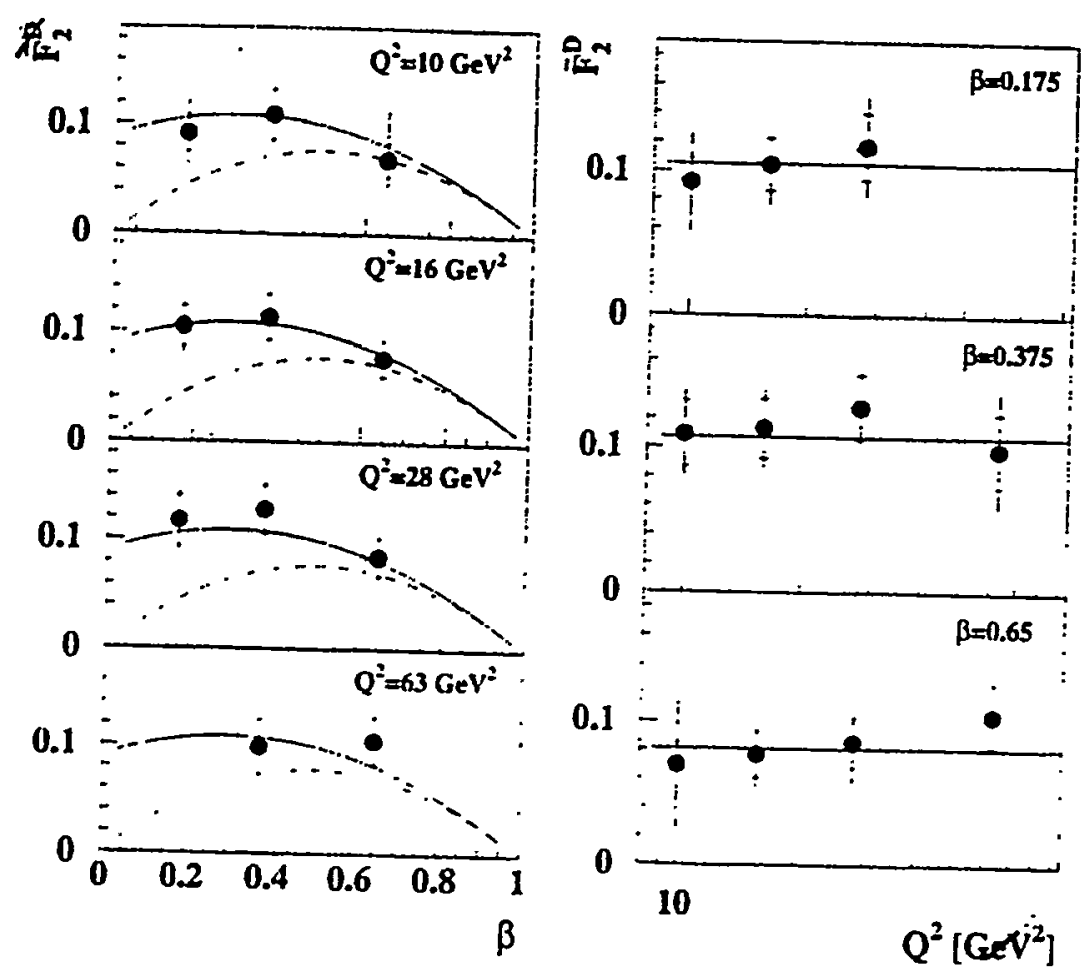

Fig. 5. The results of $F_{2}^{D}\left(\beta, Q^{2}\right)$ compared to the parametrisation discussed in the text, indicated by the full line, and the $\beta(1-\beta)$ hard contribution, indicated by the dashed line. The inner error bars show the statistical errors, the outer bars correspond to the statistical and systematic errors added in quadrature. The systematic errors combine in quadrature the fits of the $\chi_{\mathbb{P}}$ dependence due to each of the systematic checks discussed in the text. Note that the overall normalisation is arbitrary and is determined by the experimental integration limits over $\chi_{\mathbb{P}}\left(6.3 \cdot 10^{-4}<\chi_{\mathbb{P}}<10^{-2}\right)$. The data include an estimated $15 \%$ contribution due to double dissociation. 


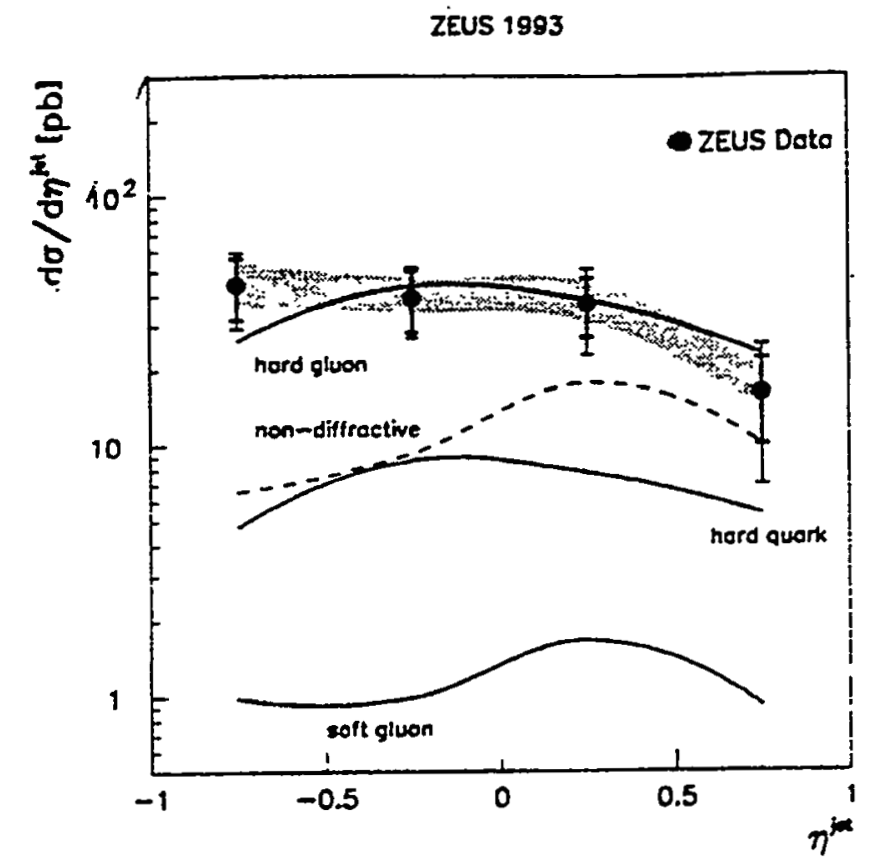

Fig. 6. Measured differential ep cross section $d \sigma / d \eta^{\mathrm{jet}}(\eta \underset{\max }{h a d}<1.8)$ for inclusive jet production for $\mathrm{E}_{T}^{j e t}>8 \mathrm{GeV}$ in the kinematic region $Q^{2} \leq 4 \mathrm{GeV}^{2}$ and $0.2<y<0.85$ (dots). The measurements are not corrected for the contributions from non-diffractive processes and double dissociation. The inner error bars represent the statistical errors of the data, and the total error bars show the statistical and systematic errors - not associated with the energy scale of the jets - added in quadrature. The shaded band displays the uncertainty due to the energy scale of the jets. For comparison, POMPYT predictions for single diffractive jet production $\left(e+p \rightarrow e+p+j e t+X_{r}\right)$ using the DL flux factor for direct plus resolved processes for various parameterisation of the pomeron parton densities (hard gluon, upper solid line; hard quark, middle solid line; soft gluon, lower solid line) are also shown. The GS-HO photon parton densities have been used in POMPYT. The contribution from nondiffractive processes is exemplified by the PYYHIA predictions using MRSD (GRV-HO) for the proton (photon) parton densities (dashed line). 


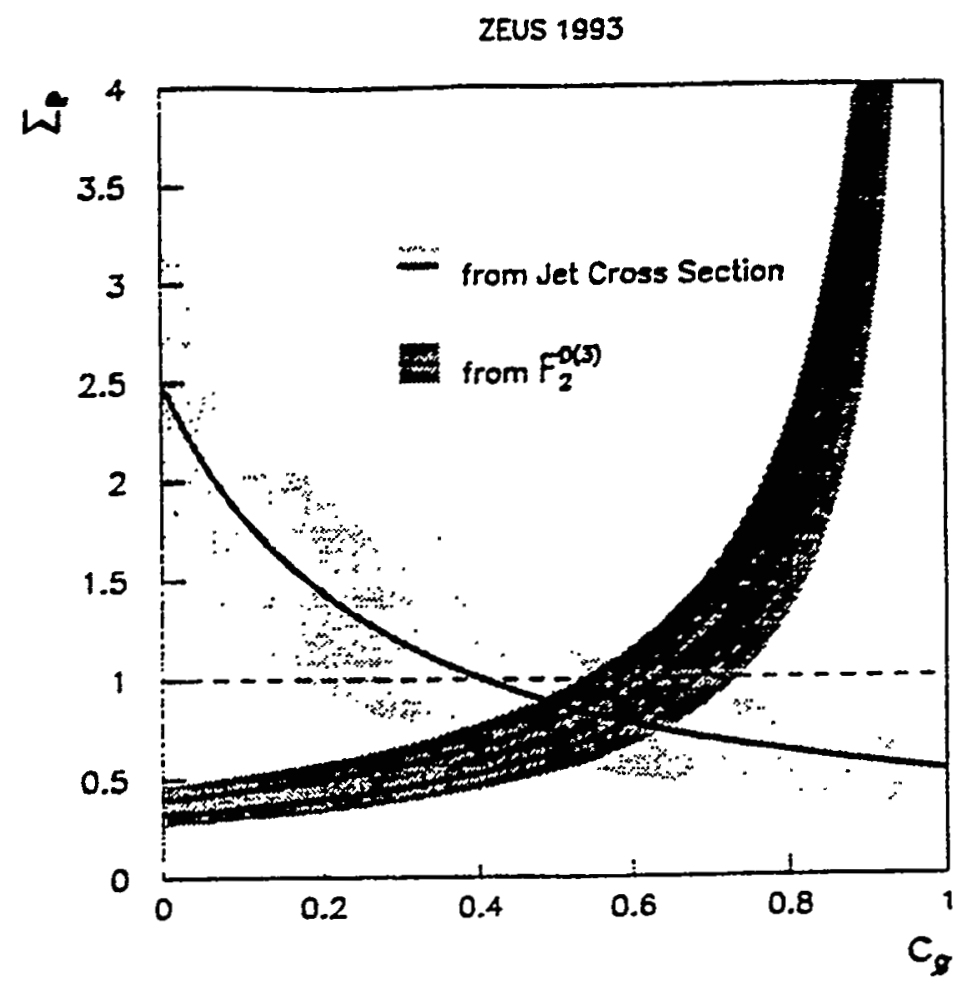

Fig. 7. The plane of the variables $\Sigma_{p}$ (momentum sum) and $\mathrm{c}_{\mathrm{g}}$ (relative contribution of hard gluons in the pomeron). The thick solid line displays the minimum for each value of $c_{g}$ obtained from the $\chi^{2}$ fit (the shaded area represents the 1 or band around these minima) to the measured $d \sigma / d \eta^{\text {jet }}\left(\eta_{\max }^{\text {had }}<1.8\right)$ using the predictions of POMPYT. The constraint imposed in the $\Sigma_{\mathrm{p}}-c_{\mathrm{g}}$ plane by the measurement of the diffractive structure function in DIS $\left(\mathrm{F}_{2}^{D(3)}\right)$ for two choices of the number of flavous (upper dot-dashed line for $\Sigma_{\mathrm{pq}}=0.32$ ) is also shown. The horizontal dashed line displays the relation $\Sigma_{\mathrm{p}}=1$. 


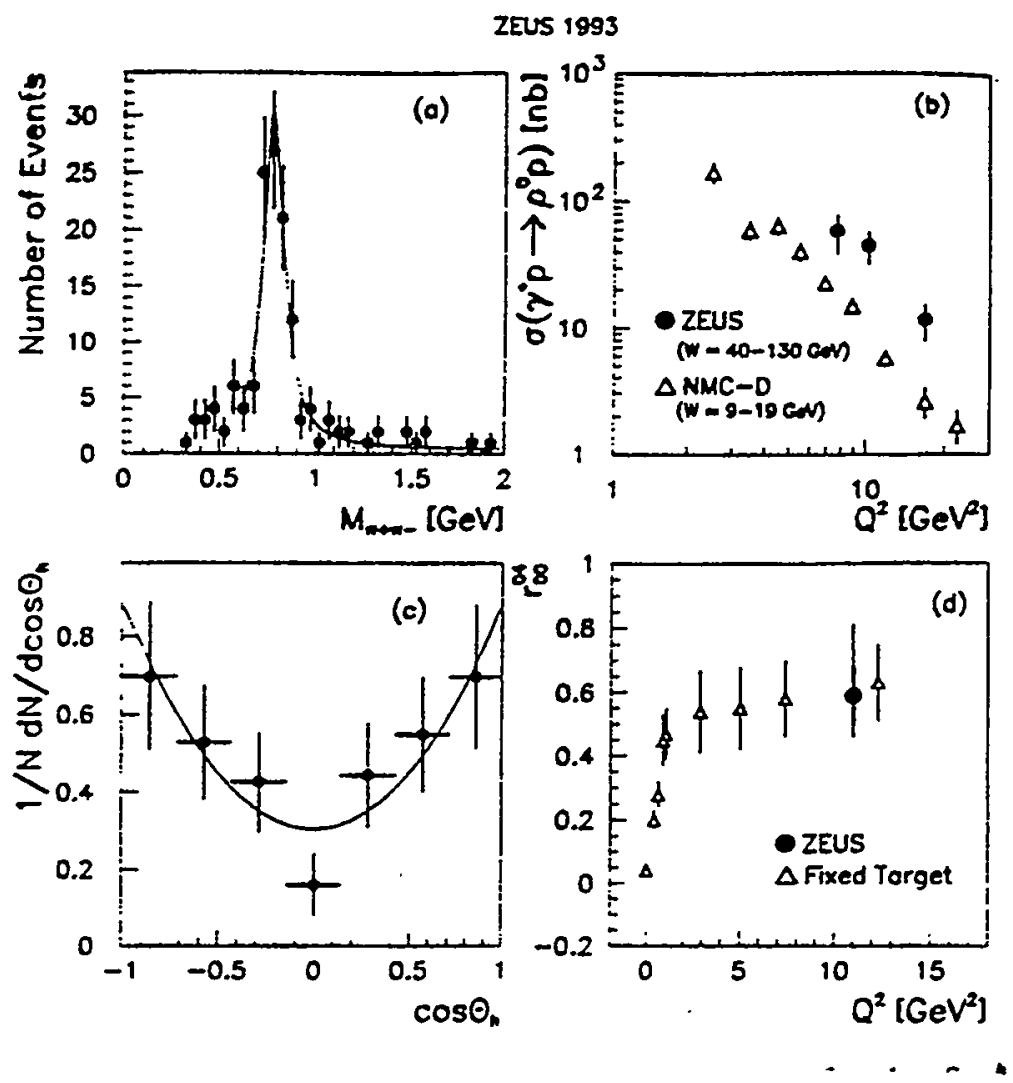

Fig. 8. (a) The $\pi^{+} \pi^{-}$invariant mass distribution for the final sample of events; the curve is a maximum likelihoodc fit with a non-relativistic Breit-Wigner distribution plus a flat (4\%) background; (b) the cross section for $\gamma^{*} p \rightarrow p^{0} p$ as a function of $Q^{2}$ for $0.0014<\chi<0.004$. Also shown are data from the NMC experiment; the errors shown are just the statistical errors. The ZEUS (NMC) data have an additional 31\% (20\%) normalisation uncertainty (not shown); $\odot$ the $\cos \theta_{\mathrm{h}}$ distribution for the decay $\pi^{+}$, in the s-channel helicity system, corrected for acceptanc e, for $\pi^{+} \pi^{-}$pairs in the mass range $0.6-1.0 \mathrm{GeV}$; (d) the $p^{0}$ density matrix element, $r_{(x)}^{04}$, compared with results from fixed target experiments as a function of $Q^{2}$. The thick error is the statistical error and the thin error is the systematic error added in quadrature. 


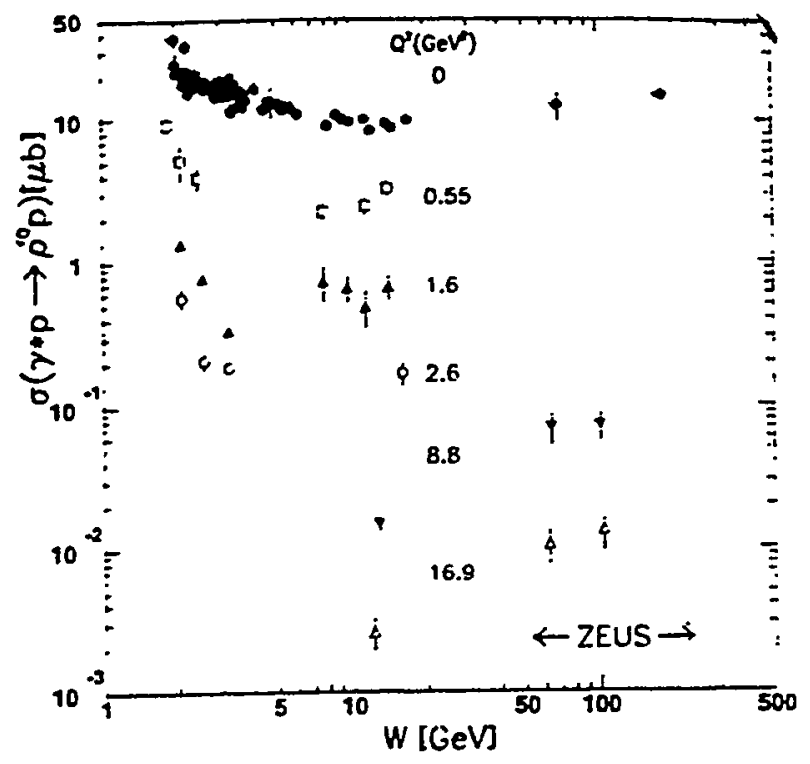

Fig. 9. The $\gamma^{*} p \rightarrow p^{0} p$ cross section as a function of $\mathrm{W}, \gamma^{*} p$ centre of mass energy, for several values of $Q^{2}$ low energy data $(\mathrm{W}<20 \mathrm{GeV}$ ) come from fixed target experiments. The high energy data ( $\mathrm{W}>50 \mathrm{GeV}$ ) come from the ZEUS experiment and the present analysis. The ZEUS data at $Q^{2}=8.8$ and $16.9 \mathrm{GeV}^{2}$ have an additional $31 \%$ systematic normalisation uncertainty (not shown). 


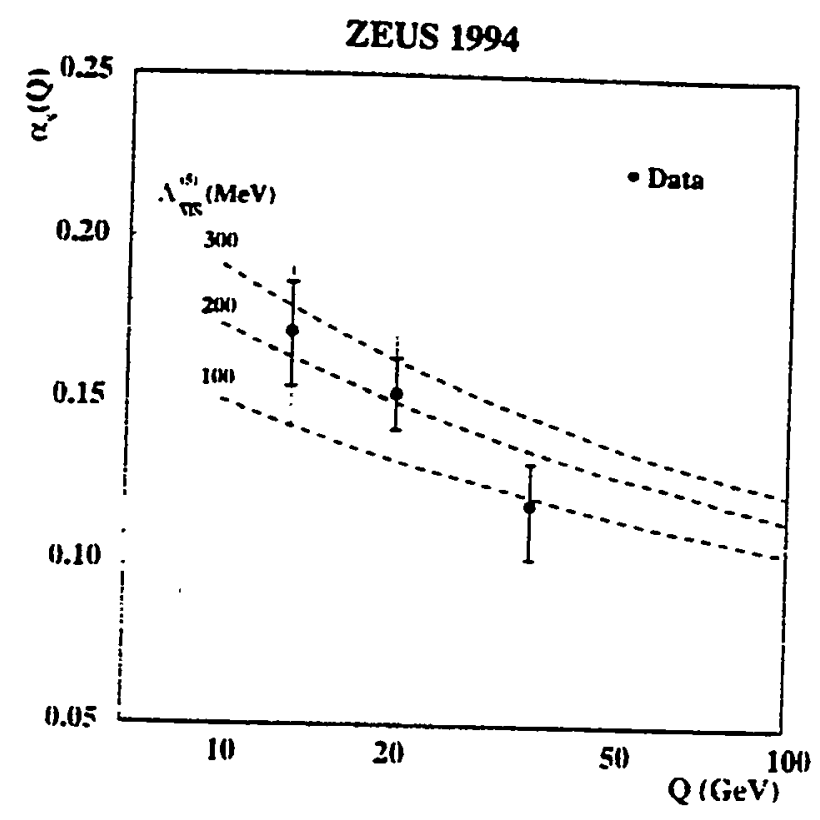

Fig. 10. Measured values of $\alpha_{s}(Q)$ for three different $Q^{2}$ regions. The statistical error corresponds to the inner bar and the thin bar reflects the statistical and systematic error added in quadrature. Note that the systematic errors are strongloy correlated. The dashed curves represent $\alpha_{s}$ with $\mathrm{A} \frac{(5)}{M S}=100,200$, and $300 \mathrm{MeV}$. 


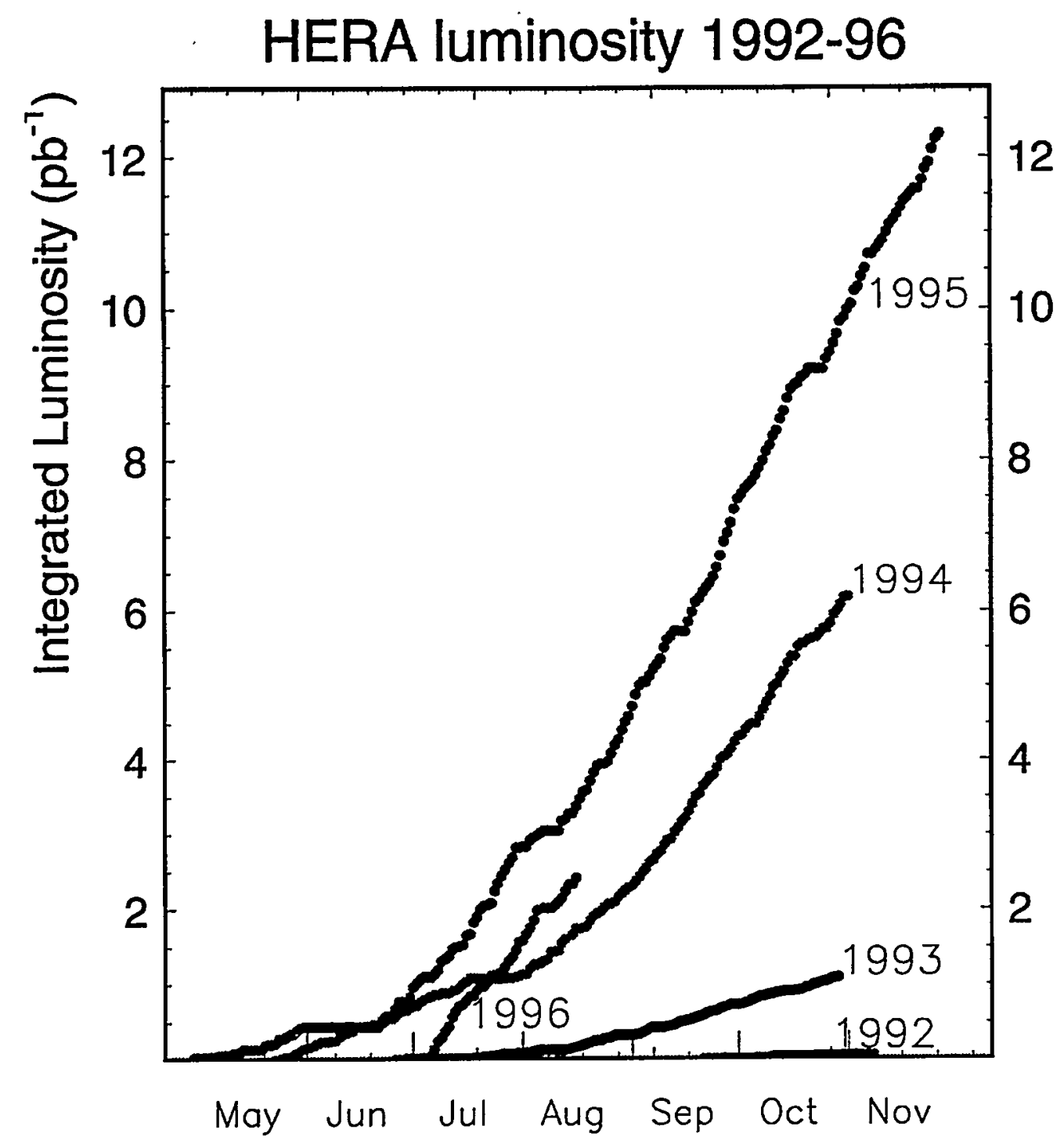

Fig. 11. HERA luminosity history 


\section{A. 6 BNL AGS Partial Snake Magnet Experiment}

The 1996 running of the partial snake experiment at Brookhaven, E880, will focus on new methods of crossing spin resonances. We had successin both 1994 runs with the tune jump method for some resonances, but not adaquate success at all resonances. The fast quadrupolesused for tune jumps were removed in July. We will next concentrate on two methods. One is decoupling the horizontal and vertical betatron oscillations which appear to make the spin resonances worse when they coincide. The other is changes in radius at the resonance energies.

The beam intensity will be higher in the coming run than in the past. It will be even higher when polarized beam is injected into RHIC. This is primarily because of more bunches from the booster being loaded into the AGS. The increased beam will be a big advantage for the beam position and phase space diagnostics measurements. We must improve the polarimeter to handle the increased intensity. This will be done in two ways. Two of the 11 scintillation counters on each polarimeter arm will have new phototubes of 10 stages instead of 14 stages, and high current tube bases will be used. These are being prepared at Argonne. The other change is to run with carbon fiber target having lower mass than the fishline target. This was tested in the last run. With carbon, there is no pure hydrogen signal, so the polarimeter becomes a relative polarimeter.

(D. Underwood) 


\section{I.B EXPERIMENTS IN PLANNING OR CONSTRUCTION}

\section{I.B.1 RHIC Spin Physics Program and STAR Detector}

In early summer, an Electromagnetic Calorimeter (EMC) task force was formed for bringing the EMC project through a successful technical review on the start of construction. The task force, working closely with the EMC project leader, is to develop a reviewable project outline, solid technical cost/schedule, work plan, identify problem areas, and form an effective team in order to get the job done. The task force was chaired by Lee Schroeder, LBNL, met at Argonne (July 19th) to review EMC status, in particular - EMC FTE's activities at ANL, and cost roll-up (first estimate, "Straw-man"). Since then, there have been weekly phone conferences and Tuesday morning video conferences.

During the STAR collaboration meeting in August, some of the important issues, which were the major focus of the meeting, included examining the significance of the STAR Spin program in a world perspective and the role of spin physics in the overall STAR program, reviewing the manpower on the STAR Additional Experimental Equipment (AEE), and charting a course to bring these projects through the final review process to construction.

The report of a BNL Director's committee, which reviewed the RHIC spin program, describes that RHIC will provide an excellent opportunity for the study of proton spin structure and that STAR, in its upgraded form, is well matched to a strong spin physics program at a polarized RHIC. Now that the RHIC spin program has once again received a strong endorsement it was an appropriate time to re-examine the role of spin physics as an integral part of the STAR physics program.

An important focus was to examine the manpower and effort on the STAR AEE (beyond that required for the STAR baseline) and insure that it is sufficient to conclude that the final review process in a timely way, allowing the AEE projects endorsed by the NSAC subcommittee earlier this year (SVT, EMC, TOF) to proceed to construction.

In September, a Memorandum of Understanding between the Institute of Physical and Chemical Research (RIKEN), Japan, and Brookhaven National Laboratory concerning the collaboration on the spin physics program at the RHIC was signed. RIKEN will provide funding for the construction and maintenance costs of accelerator hardware and experimental apparatus for the spin physics program which amounts to about $\$ 20 \mathrm{M}$. This was a huge breakthru for spin physics at BNL.

In October, a STAR visit to DOE NP took place in order to prevent a complete lapse of funding for STAR EMC. "Day One Physics with 25\% Coverage" was stressed and funding required for FY96 to FY2000 was presented.

The BNL NP and HEP management reminded the RHIC spin collaboration that the physics goals of STAR and PHENIX require a polarimeter system accurate to $\triangle P / P=$ $\pm 5^{\circ}$ (absolute calibration, not just calibration) and requested to organize a Polarization 
Measurement Working Group (PMWG) to investigate and formulate a proposal to achieve the beam polarization measurements with an accuracy mentioned above. The PMWG was formed in the fall and several discussion meetings were held.

\section{(A. Yokosawa)}

The 1996 running of the partial snake experiment at Brookhaen, E880, will focus on new methods of crossing spin resonances. We had success in both 1994 runs with the tune jump method for some resonances, but not adequate success at all resonances. The fast quadrupole used for tune jumps were removed in July. We will next concentrate on two methods. One is decoupling the horizontal and vertical betatron oscillations which appear to make the spin resonances worse when they coincide. The other is changes in radius at the resonance energies.

The beam intensity will be higher in the coming run than in the past. It will be even higher when polarized beam is injected into RHIC. This is primarily because of more bunches from the booster being loaded into the AGS. The increased beam will be a big advantage for the beam position and phase space diagnostics measurements. We must improve the polarimeter to handle the increased intensity. this will be done in two ways. Two of the 11 scintillation counters on each polarimeter arm will have new phototubes of 10 stages instead of 14 stages, and high current tube bases wll be used. These are being prrepared at Argonne. The other change is to run with carbon fiber target having lower mass than the fishline target. This was tested in the last run. With carbon, there is no pure hydrogen signal, so the polarimeter becomes a relative polarimeter.

(D. Underwood)

\section{I.B.2 MINOS-Main Injector Neutrino Oscillation Search}

The Soudan group, which previously had been working on a proposal for a Fermilab neutrino oscillation experiment (P822) using Soudan 2, has joined a new collaboration to build a large new detector in the Soudan mine to study neutrino oscillations. The collaboration includes Argonne, Boston College, Caltech, Columbia, Fermilab, Houston, Indiana, ITEP, Lebedev, Livermore, Minnesota, Oak Ridge, Oxford, Rutherford, Stanford, Sussex, Texas A\&M, Tufts, and Western Washington. The MINOS Collaboration proposes to conduct a search for $v_{\mu} \rightarrow v_{\tau}$ and $v_{\mu} \rightarrow v_{e}$ oscillations using a new $v_{\mu}$ beam from the Fermilab Main Injector with energies well above the $\tau$ production threshold. Oscillations will be detected by the comparison of signals in a 'near' detector at Fermilab and a 'far' detector situated $730 \mathrm{~km}$ away. A new $10 \mathrm{kton}$ detector will be built at Soudan to allow the exploration of oscillation parameters down to $\Delta \mathrm{m}^{2} \approx 0.002 \mathrm{eV}^{2}$ and $\sin ^{2}(2 \theta) \approx 0.01$. In addition the existing, much finer grained but 
smaller, Soudan 2 detector will provide an independent check of any potential signal with $\sin ^{2}(2 \theta)$ larger than $\approx 0.1$.

In mid 1995, a HEPAP subpanel on neutrino oscillations, chaired by Frank Sciulli, compared the capabilities of the Fermilab MINOS experiment with along baseline proposal at Brookhaven (BNL889). The subpanel's recommendation was that MINOS should be supported and that BNL889 should not. They gave three reasons: 1) The combination of MINOS with COSMOS, a short baseline experiment in the same neutrino beam, will be cost effective; 2) MINOS will have higher event rates, a larger variety of signals, and the possibility of a Narrow Band Beam (NBB), and 3) MINOS will have greater "discovery potential" for $\Delta \mathrm{m}^{2}<0.02 \mathrm{eV}^{2}$.

The discovery potential was defined by the subpanel as the region of parameter space in which one signal has $99.9 \% \mathrm{CL}$ or greater and a second signature has $95 \% \mathrm{CL}$ or greater. Each experiment was asked to show its discovery potential. This calculation depended crucially on a full understanding of systematic errors in all tests. It quantitatively depended on the power of the second best test. The MINOS discovery potentials for $v_{\mu} \rightarrow v_{\tau}$ is shown in Figure 2. Three curves are shown for differing assumptions about the systematic error for the disappearance signature. Discovery potential for $v_{\mu} \rightarrow v_{e}$ is better by about a factor of 5 in $\sin ^{2}(2 \theta)$.

The MINOS proposal describes a $10 \mathrm{kton}$ reference detector which can be built using well understood technology. It will be built in a new hall at the Soudan mine, adjacent to the existing hall containing the Soudan 2 detector. The detector is designed to obtain measurements of muon momentum by range and/or curvature in magnetized steel, and to provide calorimetric measurements of hadronic and electromagnetic energy. The reference detector is a 36-m long, 8-m diameter sandwich of 4-cm thick octagonal steel plates separated by $2-\mathrm{cm}$ gaps containing the active detectors. A total of 600 such planes constitute the mass of $10 \mathrm{kton}$. A coil running through a central hole will produce a toroidal magnetic field of $\sim 1.5$ Tesla.

The active detector elements will be $1-\mathrm{cm}$ thick, 1-cm pitch limited streamer tubes.

Argonne has taken a major role in beam design to maximize the neutrino event rate for the NuMI project. It now appears that a focusing system with three "horn" focusing devices leads to a $50 \%$ larger event rate than has been previously assumed. The three horn design is shown in Figure 1. The neutrino fluxes for a three horn system and an improved two horn design are shown in Figure 3 along with the predictions for an ideally focused beam and a bare target (no focusing).

Argonne has undertaken an R\&D program in three areas for the MINOS detector. A limited streamer tube development program is underway to study the ideal geometry, suitability of differing gas mixtures, and to provide a basis for mass production cost estimates. An electronics development program is determining the requirements for the 480,000 channels of electronics. A steel and magnet development program is involved in 
determining the optimal configuration of the passive detector vis- $\ddagger$-vis magnetic and structural considerations.

(M. Goodman)

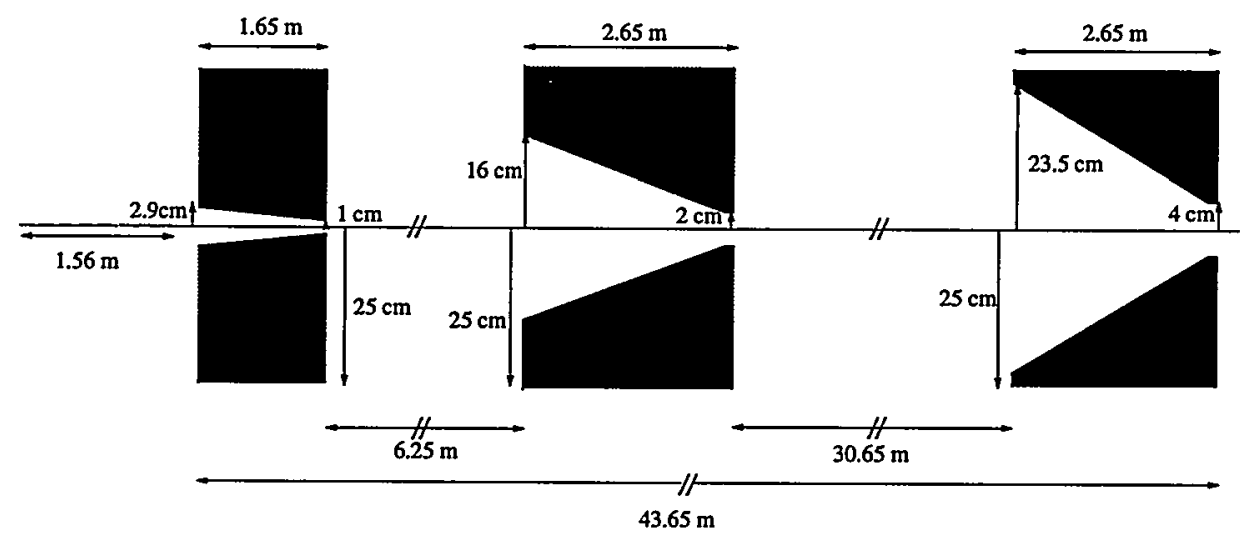

Figure 1. A three horn focusing system design. 




Figure 2. MINOS two year "Discovery level curve" for $v_{\mu} \rightarrow v_{\tau}$ oscillations. Discovery level is defined to be $99.9 \% \mathrm{CL}$ in one test and $95 \%$ in a second test. The three curves shown differ by the assumption of systematic error. The curve to left is for no systematic error. The middle curve shows the range of parameters using our present estimate of systematic errors, which is $1 \%$ for the near/far test. The curve to the right shows the parameter space using a more pessimistic assumption of $2 \%$. For parameter space to the right of each curve, we would be able to discover $v_{\mu} \rightarrow v_{\tau}$ neutrino oscillations with confidence levels greater than the specified values. 


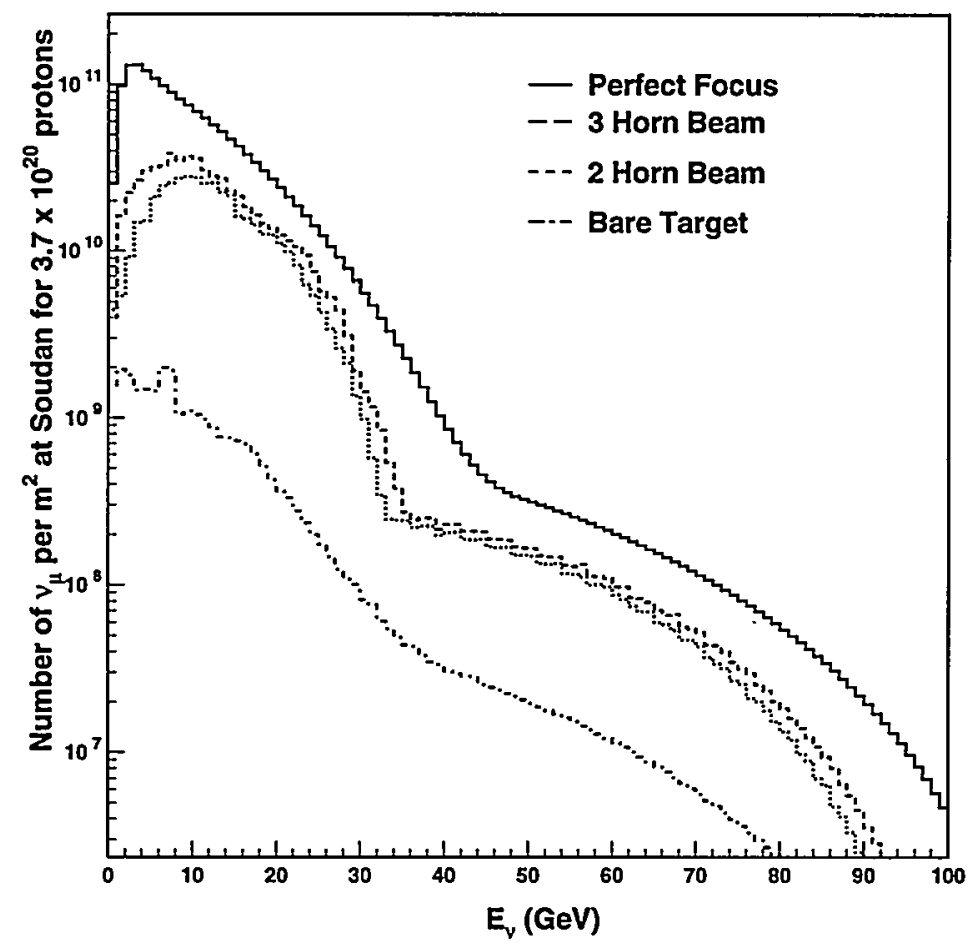

Figure 3. Comparison of neutrino fluxes at Soudan 2 for ideal focusing, a two horn design, a three horn design, and a bare target.

\section{I.B.3 ATLAS Calorimeter Development}

\section{Overview of ANL LHC Related R\&D Programs}

The second half of 1995 has seen significant progress in the ATLAS detector R\&D program. Within the mechanical program for the TileCal calorimeter, we have completed fabrication of the die and stamping of all master plates to be used in the assembly of Barrel Module 0, have completed the design and fabrication of a prototype stacking fixture for use in submodule assembly and fabricated a gauge plate for use in master plate inspection. In addition, we manufactured 80 master plates using laser cutting to meet the schedule for stacking 2 submodules at CERN in November and sent a technician to CERN to participate in this test. No significant problems were encountered in this test. The laser cut plates have also provided a cost reference point for the comparison of the two fabrication approaches and demonstrates the significant cost saving which may be obtained by die stamping the master plates. Beam tests of the test modules continued in July of this year and we contributed both effort on shift and to the data analysis in which 
we have concentrated on understanding the light output of the calorimeter and in particular the light from those segments using for the Hamamatsu R5900 photomultiplier tubes. In addition, we have begun preparations for the 1996 testbeam run and in R. Stanek have one of the two run coordinators for the 1996 period. Trigger hardware provided by Argonne was also tested in a beam in August 1995. Although extensive problems were encountered in other parts of the hardware and software being tested, the trigger supervisor built by Argonne functioned as designed. Finally, we held a 3 day ATLAS physics workshop at Argonne. This was quite successful in bringing together many US collaborators to discuss physics rather than detector technology.

(J. Proudfoot) 


\section{I.C DETECTOR DEVELOPMENT}

\section{I.C.1 CDF Detector and DAQ Electronics Development}

\section{a. Upgraded Shower Max Read out Electronics}

There was a change in personnel during this period as our graduate student, Marcus Hohlmann, completed his term with Argonne. Since October, Karen Byrum and Larry Nodulman have taken over the project.

We still await delivery from CDF for a QIE chip mounted on a test board. In the meantime, we have continued studying the linearity, gain and noise characteristics of the Harris Semi-Conductor CA3280 series chip. This transconductance operational amplifier was to be the candidate for the read out electronics of the shower max detector.

During this time, we made real progress in reducing the noise in our test setup by roughly a factor 3 . With this reduction, comparisons between our test setup and the readout electronics at CDF are almost comparable. We then found however, the noise characteristics of the CA3280 chip to be unacceptable, introducing roughly a factor 10 more noise into the system than without the chip. As a result of these noise studies, we are now investigating other solutions.

(K. Byrum)

\section{b. CDF Central Tracking Upgrade}

In June, 1995 the Fermilab Physics Advisory Committee requested that CDF develop an integrated tracking plan for Tevatron Collider Run 2. As part of this, a plan for replacing the existing Central Tracking Chamber (CTC) was requested. Beginning in July, two alternative proposals were developed: a straw tube tracking chamber based on the existing SDC Outer Tracker design and an open cell tracker similar to the existing CTC but with a factor of four finer wire spacing.

The straw tracker proposal was developed by a subset of the existing CDF institutions: Argonne, Duke University, Fermilab, Johns Hopkins University, University of Michigan, University of Pennsylvania, and Rockefeller University. This collaboration formed around the original group that had proposed a straw tracker option for an intermediate tracking detector for CDF. This proposal has been described in a previous semi-annual report.

The straw tube tracker proposal was presented to the CDF collaboration on 20 September 1995. Subsequent to review by a specially constituted Technical Review panel and the Tracking Godfather committee for CDF, the straw tracker option was chosen over the open cell geometry chamber as the preferred replacement for the existing 
CTC. The tracking godparents identified a few items to be addressed in the Central Straw Tracker (CST) design and work was begun in October, 1995 to address these problems. These included increasing the straw tube diameter from $4 \mathrm{~mm}$ to approximately $6 \mathrm{~mm}$ and defining the number of layers in the axial view to be 12 per superlayer rather than 8 . These two changes improved the pointing accuracy of the detector and gave better assurance of efficient track segment linking during reconstruction. Alignment of the straws was also suggested as being in need of improvement. The Argonne group worked with Duke and Fermilab to improve the mounting scheme for straw bundles to provide a more easily achievable alignment to the required precision of 50-100 microns per wire.

The efficiency and speed with which pattern recognition was accomplished in the CST were also questioned early after the tracking decision was made. Both Barry Wicklund at Argonne and Seog Oh at Duke University provided crucial work to demonstrate that pattern recognition and track reconstruction, while being handled fundamentally different from that of an open cell tracker, were readily and perhaps more efficiently handled in the CST.

At the end of the calendar year the status of the CDF central tracking upgrade was a first pass design of the CST layout (see figure ??) and uncertainty over the budget for the upgrade. Given the budget uncertainty and schedule needed for completion, the competition for tracker choice for Run 2 will most likely be reopened. Whether either the CST or the open cell tracker has either a cost or schedule advantage will be re-examined. A decision will be forthcoming in March or April, 1996. 




Figure 1. A possible layout for one of four pairs of axial/stereo superlayers as developed by physicists, engineers, and designers within the Argonne High Energy Physics Division. The straws which contain the sense wires are bundled and contained within the modules illustrated in the layout. The locating pin is used to provide accurate $( \pm 100$ micron) placement of the module onto the mounting cylinder.

(R. Wagner)

\section{I.C.2 ZEUS Detector Upgrades}

ZEUS collaborators from Argonne continued to study upgrades to the ZEUS Barrel Calorimeter. Studies of the performance and feasibility of a wire and pad-based Barrel Hadron Electron Separator (BHES) were completed with data taken in the BNL test beam. Also, lab and test beam studies of a scintillator-based pre-shower detector were 
performed, resulting in a proposal for the addition of a Barrel Presampler (BPRE) to the ZEUS Detector.

\section{a. Barrel Hadron Electron Separator (BHES)}

The BHES testbeam results were submitted to Nuclear Instruments and Methods and will appear in an early 1996 issue.

At DESY, one complete module (4 skis) was installed into an accessible BCAL module and read out along with the ZEUS 1995 data. The objective of this test was to try to identify electrons and pions from ep events in ZEUS. This data is currently being analyzed.

\section{b. Barrel Presampler (BPRE)}

During the first 6 months of 1995, lab tests of various configurations of scintillator tiles with fiber readout were done, leading to a practical design for a barrel presampler detector. Subsequently, three versions of the optimum tile configuration were tested in August, 1995 at the BNL test beam. Figure 1 shows the response of the calorimeter versus the presampler response for a) incident electrons of various beam momenta passing through a $1.5 \mathrm{X}_{0}$ absorber, and b) $5 \mathrm{GeV}$ electrons through various absorber thicknesses. Clearly, the relationship between the calorimeter and presampler response is more strongly dependent on the amount of absorber in front of the calorimeter than it is on the energy of the incident electrons. It is this feature of the presampler/calorimeter signal relationship that allows particles and jets to be corrected independently of energy, but most optimally, in a region in which the amount of dead material is relatively well known. Figure 2 shows the uncorrected and corrected calorimeter energies using the presampler from the BNL test beam data. Knowledge of the amount of dead material clearly makes this correction more effective. Finally, Figure 3 shows the improvement in resolution obtained with the presampler used to correct the calorimeter energy measurement. The dashed line represents the resolution obtained with no absorber in front of the calorimeter.

Also tested at BNL was the capability of the presampler to separately identify electrons and pions. A factor of 3 improvement over the separation capabilities of the calorimeter only measurement was obtained.

The results of these prototype tests combined with Monte Carlo studies led to the design of a complete barrel presampler detector and a proposal to build and install this device in the ZEUS Detector.

A schedule for approval of the barrel presampler project was made, with the goal of obtaining final DESY Physics Research Committee (PRC) approval at its January, 1996 meeting. Based on this schedule, representatives of the US ZEUS group asked the US Department of Energy (DOE) in September, 1995, for money to begin construction of part of the ZEUS barrel presampler. Some funds were allocated at that time and it was agreed that after final approval by the ZEUS collaboration and the DESY PRC, a request for full funding of the complete barrel presampler would be considered by the DOE. 
The proposal was first presented to the ZEUS collaboration at a meeting in October, 1995. It was evaluated by internal referees, modified according to their recommendations, and subsequently approved by the ZEUS Executive Committee. The final proposal was submitted to the DESY PRC for consideration at its January 16-17, 1996 meeting.

The project will be implemented by the following groups : Argonne, the University of Iowa, and Virginia Polytechnic Institute and State University (VPI) in the US; the Weizmann Institute in Israel; and DESY in Germany. In addition, a substantial amount of help and cooperation from several other ZEUS groups who were responsible for the construction, installation, and operation of the F/RCAL presampler is anticipated.

(S. Magill)



Figure 1. a) BCAL measured energy (Ecal) versus presampler signal in mips for several momenta electrons through an absorber thickness of $1.5 \mathrm{X0}$; and b) BCAL measured energy (Ecal) versus presampler signal in mips for $5 \mathrm{GeV}$ electrons through various absorber thicknesses. The lines represent the best straight line fits to the data. The fit parameters are used to determine the correction parameters to the measured calorimeter energy. 

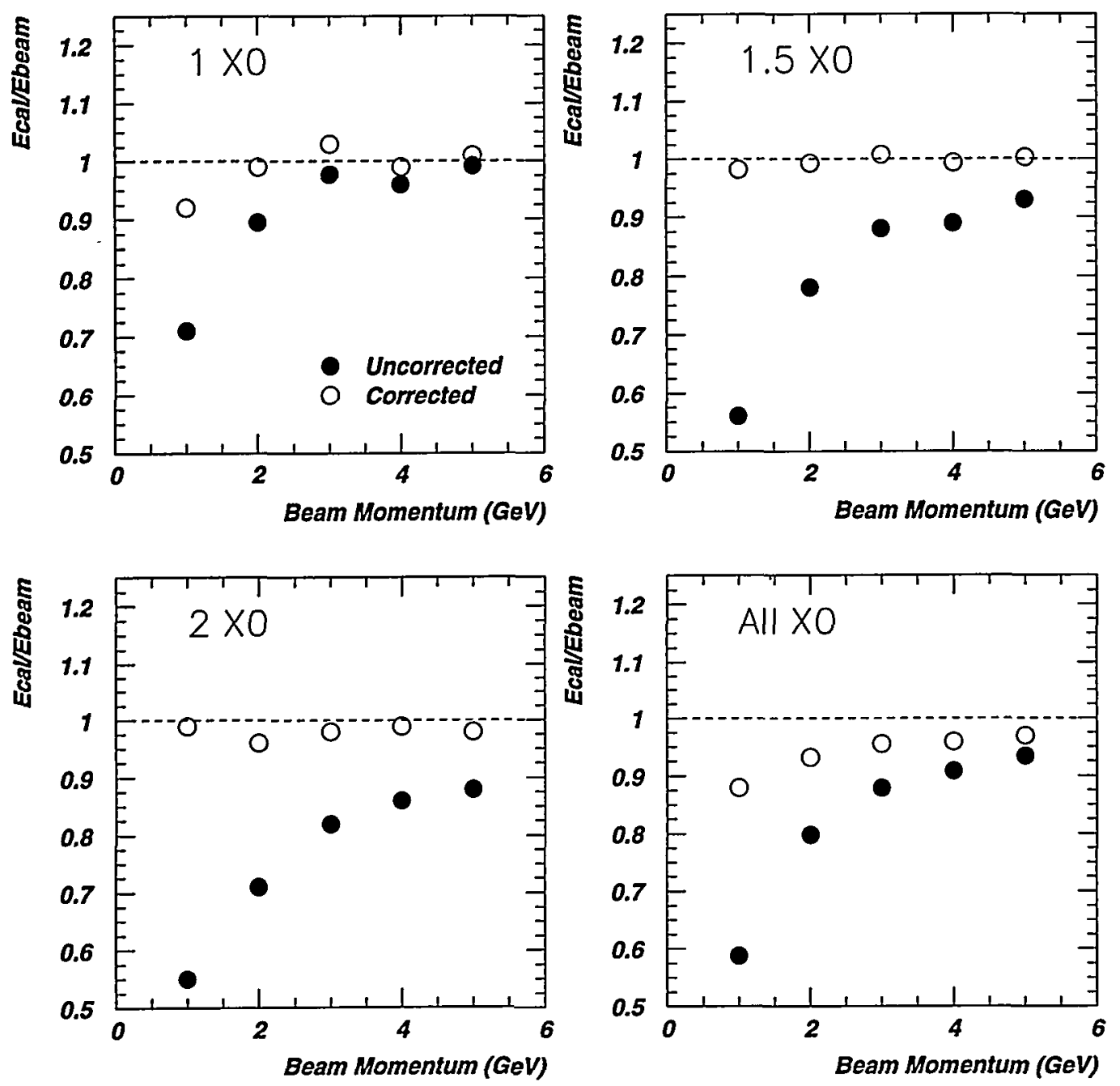

Figure 2. The measured BCAL energy ratio, E/E0 (= E/Ebeam), for $1 \mathrm{X} 0,1.5 \mathrm{X} 0,2 \mathrm{X} 0$, and All X0 radiators. In each plot, the uncorrected points (solid) are compared with the corrected points (open) using the presampler and calorimeter data for the indicated thickness of absorber. The indication "All $\mathrm{XO}$ " means that all of the data have been combined. 

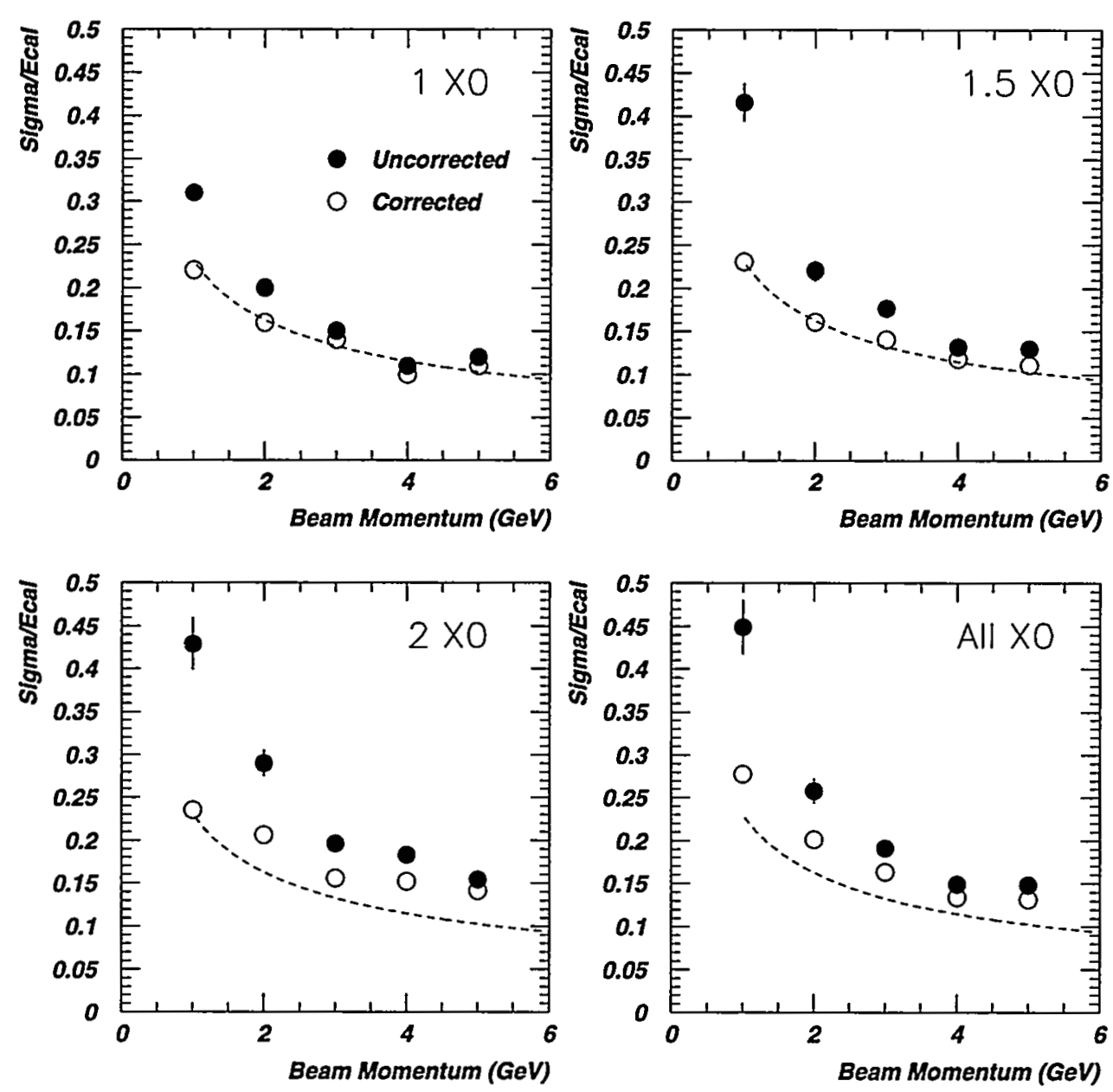

Figure 3. The measured BCAL resolution Sigma/Ecal for $1 \mathrm{X0}, 1.5 \mathrm{X} 0,2 \mathrm{X} 0$, and All $\mathrm{X} 0$ radiators. In each plot, the uncorrected points (solid) are compared with the corrected points (open). The dashed line represents the measured Sigma/Ecal response of the BCAL prototype module to electrons incident directly on the calorimeter with no absorber.

\section{I.C.3 STAR Calorimeter Development}

Much of the effort recently has gone into trying to obtain funding for the calorimeter. It now appears that DOE will fund $1 / 4$ of the barrel as additional equipment for RHIC detectors. Also, Penn State and Michigan State are talking to NSF about funding the endcap detector. 
The head of the calorimeter subsystem, from Argonne, presented one of the three talks on STAR, at the BNL/RHIC review of RHIC Spin Physics. Also, a review of the RHIC spin program was given at DESY Zeuthen. This was for purposes of planning the future of DESY:

Wayne State has become much more involved in the barrel calorimeter as many of the mechanical parts will be produced there.

The planning for all aspects of the STAR calorimeter electronics was consolidated in a new version of the Requirements Document for the Electromagnetic Calorimeter collaborating institution.

(D. Underwood) 


\section{I.C.4 ATLAS Calorimeter Design}

\section{I.C.4.a. Hadron Calorimeter Mechanical Design}

The focus of the mechanical design work on ATLAS continues to be the production of full scale prototype modules for both the barrel and extended barrel regions of the detector. The prototype stacking fixture has been completed and was used in the stacking of 2 submodules at CERN in November, with a baseplate and compression plate provided by CERN. Argonne has advocated the use of die stamping for the fabrication of master plates for some time. The die was completed and used successfully for the production of about 1200 plates. The die was finished somewhat later than expected and therefore we laser cut about 80 plates for the CERN test in November. This gave us the opportunity to get a direct comparison between the two approaches and as a result we now have direct cost comparison which greatly favors die stamping. The following internal Technical notes and papers have been submitted during this report period.

1. Procedure for stacking TileCal submodules using the Argonne designed stacking fixture", N. Hill, 21 Jul 1995.

2. "Specifications for plate fabrication for the ATLAS Tile Hadron Calorimeter", N. Hill, ANL-HEP-TR-95-39.

3. "Report of "Time Saver" surface finish tests on Czech Steel samples", N. Hill and C. Keyser, ANL-HEP-TR-95-55.

\section{a.1. Stacking Fixture}

The ATLAS submodule stacking fixture was completed as designed at Argonne and the small parts shipped to CERN for the first submodule stacking tests in November. A photograph of this fixture is shown in Figure 1. A second set of heavy parts (base plate and upper platen) were fabricated at CERN to reduce shipping costs. The tests were carried out at CERN in November and one technician (K. Wood) was present to participate in the test assembly work. Two submodules were stacked using all of the production tooling. These tests were successful and the other proposed assembly collaborators were given the go ahead to fabricate duplicate tooling and begin the assembly of the respective submodule contribution. Some minor problems were discovered during the test assembly (of note was some interference on tooling required to weld on strengthening bars to the submodule) and were corrected for the follow on work.

\section{a.2. Master Plate Manufacturing}

A bid package was solicited from Argonne for the design and construction of the large die to be used in the stamping of the necessary master plates for the first barrel module ( 6 
meter). The design for these plates is shown in Figure 2. Despite the size and complexity of the piece, tight tolerances are required to minimize the fraction of uninstrumented detector.

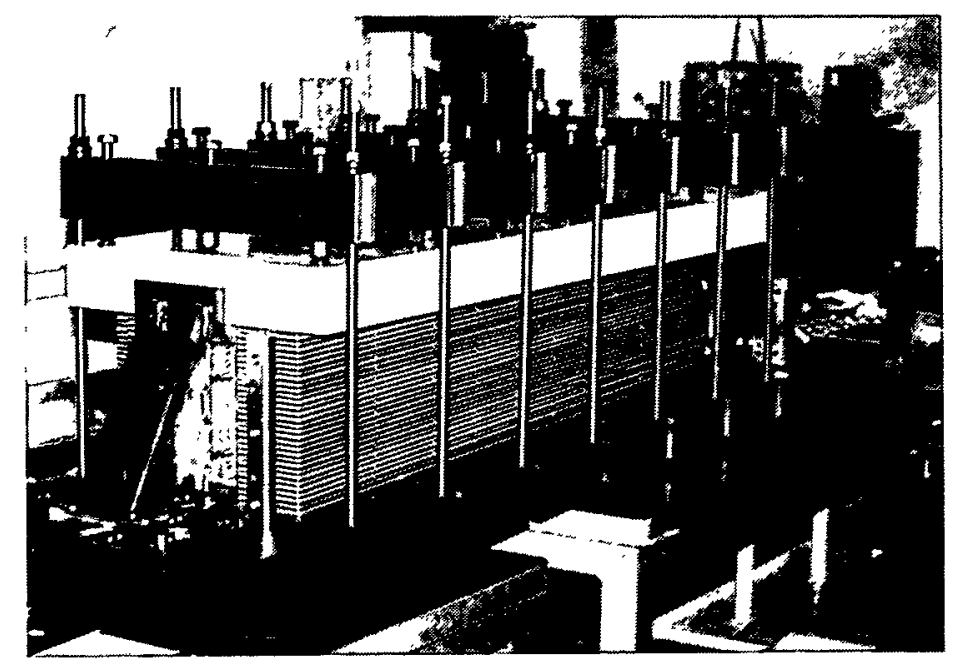

Figure 1. The stacking fixture designed at Argonne for use in the production of submodule.

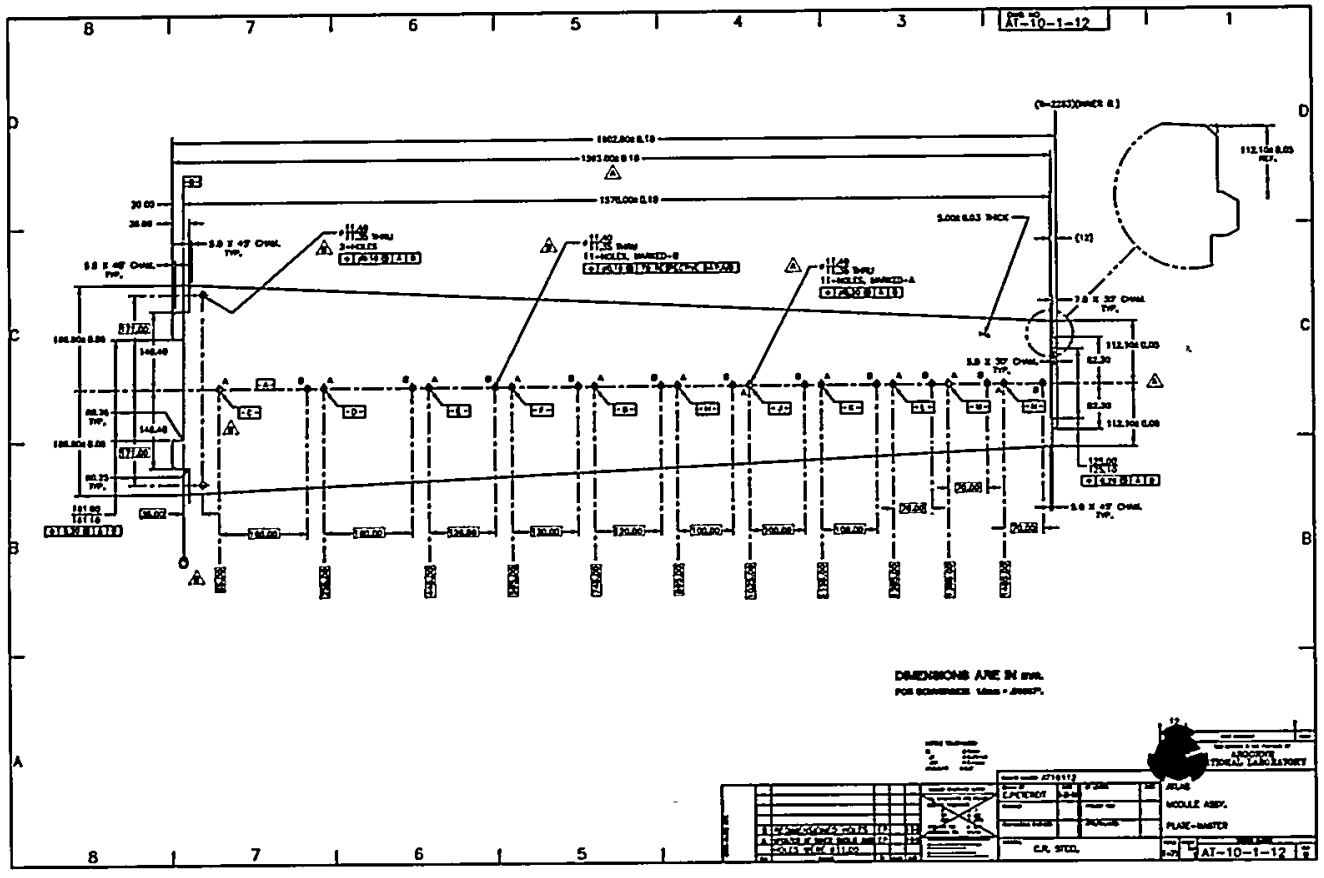

Figure 2. The keys at either end are used for location of the plate in the support girder. The holes are used to allow passage of a radioactive source for calibration of scintillators. 
The contract was let to Banner Tool and Engineering, Milwaukee, Wisconsin for the entire package including the stamping of 1200 master plates. The die was completed and tested in November and the production run of 1200 plates was carried out in midDecember. Figure 3 shows the die as setup in the press for this production run. The stamped plates were not available for the test assembly at CERN in November and 80 plates were cut in the US for that test. These plates gave a good comparison of the cost and tolerance capability of the two methods. The cost comparison for the production plates is approximately 5 to 1 with the stamped plates at high production costing less than $\$ 6$ US each. A certified inspection of the stamped plates was completed by the vendor with no discrepancies in the specified tolerances. Although the laser cut plates held the required tolerances, there were problems with blowout in the holes caused by heat concentration in the limited area of the holes. The stamped plates were packaged and shipped to the four assembly locations. These were Dubna, Russia; Barcelona, Spain; Pisa, Italy; and Prague, Czech Republic. It became apparent in performing the necessary quality control on the stamped and laser cut plates that a simple gauge was necessary to reduce the amount of inspection time. A gauge was designed at Argonne and used for that purpose. The drawings of that gauge were distributed to the other collaborating institutions.

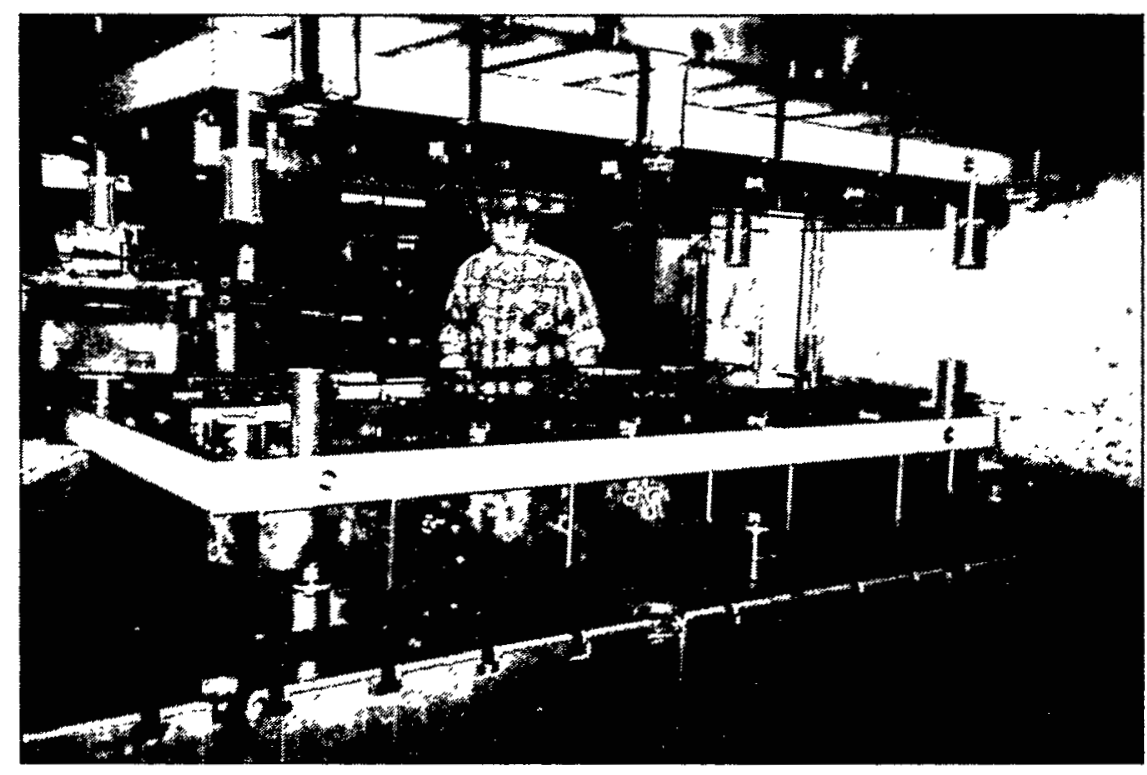

Figure 3. The stamping press and die used for production of master plates.

\section{a.3. Proof test of sub-module assembly procedures}

A prototype sub-module assembly line was set up at CERN in November for the stacking of 2 submodules. The Prague group provided the plate cleaning tanks, Barcelona 
provided the automated gluing machine, CERN provided the baseplate and compression plate for the stacking fixture (as they were too costly to ship) and Argonne provided the keys and load bars for the stacking fixture. These components were installed at CERN in the week of November 20th, with the gauge plate and master plates arriving from the US at the eleventh hour. We took the initiative to make some measurements on the plate thickness (since the steel had been shipped directly from the laser cutter) and to verify the plate profile of every master plate using the gauge plate (an operation which, without the gauge plate would have been inconceivable). Some of the critical component data were not as expected (both master plate thickness and eventual stack height came out somewhat higher than expected and some of the master plates were badly buckled) and it will therefore be interesting to learn how these two submodules compare to the remaining 20 that must be fabricated for module 0 . The tests of the procedures were also invaluable. Although the gluing machine worked flawlessly, the number of technicians required is still somewhat higher than desired. The cleaning procedures were hopelessly time consuming and at periods required 6 technicians working continuously. This is an area that we hope to improve on significantly when we build our submodules at Argonne in 1996. Overall, this was considered a great success - no major problems were encountered.

\section{a.4. Plate Surface Preparation}

During this reporting period, Argonne conducted a number of tests connected with the surface preparation of the submodule plates prior to bonding. These tests consisted of investigation of equipment, surface finish tests and roughness measurements and bond tests using the proposed adhesive for bonding. This effort resulted in an agreement to use a process called "Time-Saver" with equipment produced by a Minneapolis company of the same name being specified for the final finish of the plates.

\section{(N. Hill and J. Proudfoot)}

\section{b. Scintillator readout design tests}

The work on developing an optical model for light collection in the test module and barrel 0 module readout geometries was completed and documented as an ATLAS internal note. The principle conclusion from this study is that although splicing of the wavelength shifting fiber to clear fiber may not be necessary for the barrel detector, it is highly likely to be necessary for the inner depth segments of the extended barrel calorimeter.

In conjunction with the Presampler effort for ZEUS, a study of the light yield of the compact Hamamatsu R5600/R5900 phototube was undertaken. The tight quarters inside the drawer assembly require compact phototubes, and the current choice for ATLAS is the R5900. In fact there were eight of these phototubes mounted into one of the test modules for the September, 1995, testbeam run. Bench tests performed in the lab 
consisted of measuring the photoelectron yield, photocathode uniformity, linearity and gain. The bench tests of the R5600 indicated that the effective quantum efficiency of the tube was 501\% that of a Hamamatsu R580, and about $70-801 \%$ that of the standard Phillips XP2012 used for prior testbeam data taking. Figure __lref $\left\{t b \_p e\right\}$ verifies a decrease in photoelectron yield for the R5600 phototubes in the testbeam data. Typically we find about a $20-30 \%$ loss of light with the new tubes.
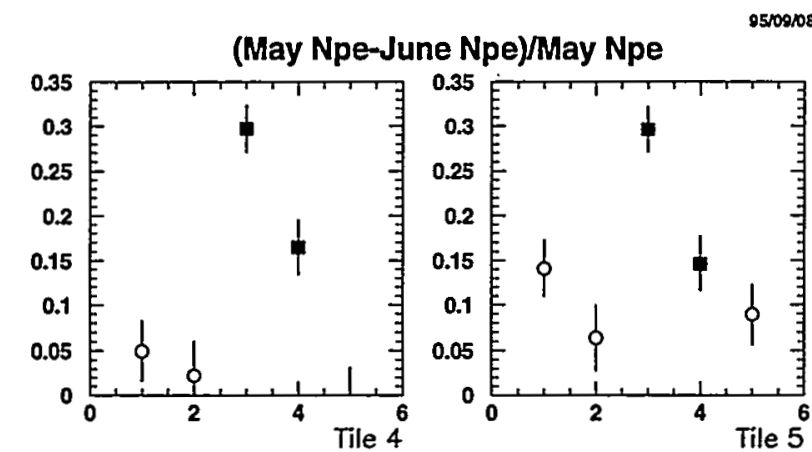

5090820.55
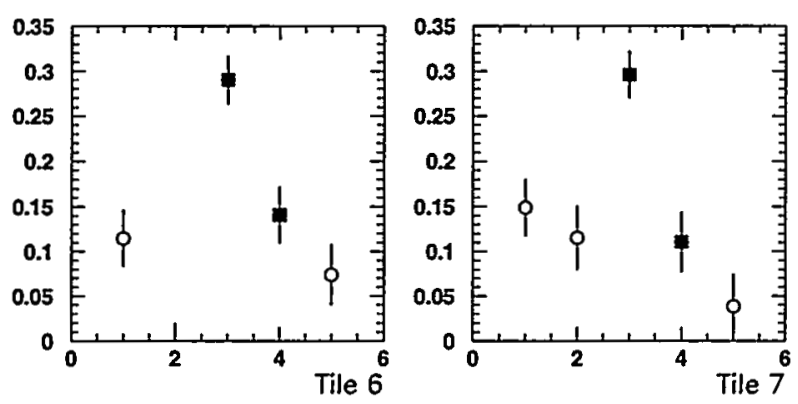

Figure 4. A representative sample of the difference in photoelectron yield of the Hamamatsu R5900 and the Phillips XP2012. June data used the Hamamatsu tubes in the towers flagged by the shaded squares.

Further work on the R5900 indicates that the linearity of the tube may not be adequate for the large dynamic range expected at the LHC. Testbeam data has shown us that the TileCal can produce 32 photoelectrons/GeV/phototube. In ATLAS, the highest energy expected to be deposited in a cell is $2 \mathrm{TeV}$. Using a series of neutral density filters, we determined that with $64 \mathrm{kPE}$, and at a gain of $10^{5}$, a lower limit, the R5900 deviates from linearity at about the $5 \%$ level.

\section{(R. Stanek)}

\section{c. Test Beam Program}

The past year saw intense activity at the testbeam at CERN where a final set of data was taken on TileCal module 3 which was implemented with the best fibers and scintillator. The stack of five test modules were scanned with beam in the standalone mode, where no liquid argon calorimeter was upstream of the TileCal. Several HEP physicists were involved in the shifts during the summer. A culmination of the test module effort resulted in an ATLAS publication documenting the performance of the calorimeter. The 
HEP division took an active role in writing the scintillator tile section, and editing the entire report.

The program for the next years' testbeam effort involves scans of Module 0 in conjunction with either the test modules or more of the 6 meter modules. To this end, the HEP division has an active role in coordinating the effort, constructing mechanical and electronics hardware and programming the devices for operation.

HEP is heavily involved in installation, software, electronics, and overall coordination. HEP has taken the role of providing the motors and motor control for the new 85 ton-capacity scanning table. The table will hold three 6-meter modules and will have degrees of freedom in $z, \theta$, and $\Phi$. Four high power motors have been investigated by HEP to service these coordinates. In addition, we will provide the interface to the drives and absolute encoders from a VME-based system. HEP was asked to provide a system which would calibrate the standard ADCs used by the test modules as well as being able to precisely calibrate regions of the FERMI shaping circuitry. We have designed a VME-based charge injection system which incorporates a 16-bit DAC which will replace the old system by the next test beam run.

(R. Stanek)

\section{d. Simulation Studies}

Work has progressed with testing and installing the most recent version of the ATLAS simulation code on Silicon Graphics workstations in the division. We have also provided support for this to other members of the ATLAS collaboration. However, at the present time we are still in the process of generating simulation data to validate the code itself rather than to study detector effects.

Independently of the Monte Carlo, we have begun studying the sampling fraction of the Tile Calorimeter by performing a numerical integration of the material along rays through the calorimeter. Figure 5, shows the sampling fraction thus measured for the test modules which were studied in the last three years and which have 18 scintillator tiles of constant radial length. The channeling effects associated with the unusual scintillator geometry are clearly visible in this plot. The corresponding plot for the prototype module which we are in the process of building is shown in Figure 6. This design has a reduced number of scintillator tiles and in addition, there are 4 four different tile sizes. The effect of these differences is to wash out the strong channeling effect. An important question is the effect of this on the constant term in the calorimeter resolution and this will be addressed in the following months using the full Monte Carlo simulation.

(R. Blair and J. Proudfoot) 

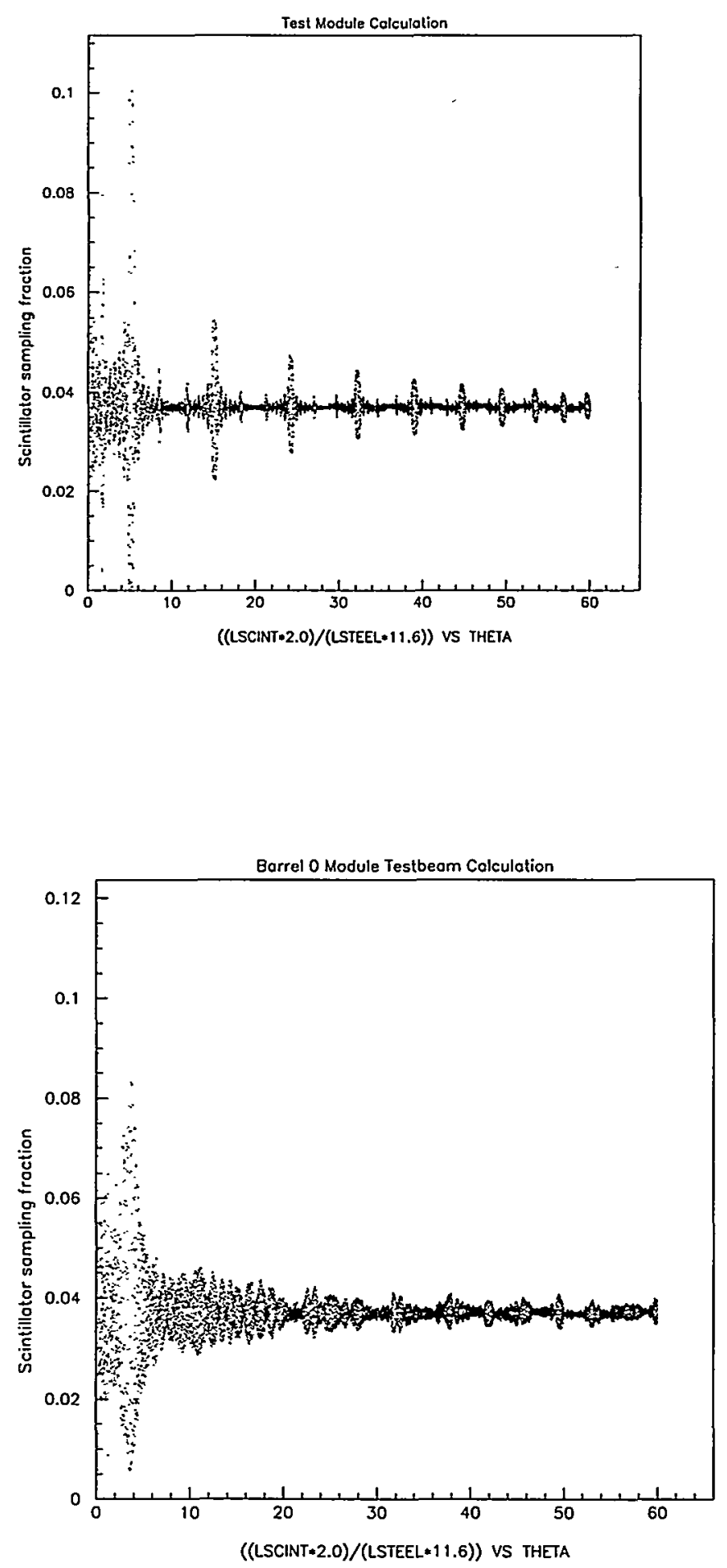

Figure 5. Variation of sampling fraction in the TileCal test modules, averaged over impact point, as a function of impact angle.
Figure 6. Variation of sampling fraction in the Barrel module 0 prototype, averaged over impact point, as a function of impact angle.

\section{I.C.5 ATLAS Detector Research \& Development}

Planning for the trigger system for the ATLAS LHC detector is currently in progress. The architectural design of the trigger has been narrowed down to three possible choices. 
A committee, which includes John Dawson of Argonne, has been appointed to oversee construction of prototypes for each of the three alternatives to assist in determining which is best. Each prototype will allow for demonstration of feasability and benchmarking of performance. Argonne and MSU are providing hardware and software for the trigger supervisor and region of interest (ROI) distribution for all three models along with additional support for evaluation and debugging of the different systems. Some of the hardware for these tests is already present at CERN. It was shipped there for testbeam use during the summer of 1995.

The three choices being considered differ in the way in which the feature extraction, or early local level 2 preprocessing, is performed. In one version the initial processing of ROI's will be performed by dedicated data driven hardware which pass their results to a network of general purpose processors. The other two models use general purpose processors to perform the initial ROI computation but differ in the way in which they are allocated to this local processing. In all three demonstrators some of the same supervisor and ROI distribution hardware will be used. After the architectural studies are complete and the architecture decision is made the next step will be to begin construction of a small scale version of the actual system chosen.

(Bob Blair)

\section{I.C.6 MINOS Detector Development}

\section{a. Introduction}

Construction of the MINOS long-baseline neutrino oscillation experiment will not begin until FY 1998, giving nearly two years to optimize the design of the detector. In addition, the MINOS collaboration is using this time to explore the possibility of improving performance by using technologies which are substantially different from those described in the MINOS proposal. Major detector development programs have been started by several MINOS collaborators to develop Resistive Plate Counters (RPC's) and tubes of liquid scintillator with fiber optics readout as possible alternatives to the limited streamer tube (Iarocci chamber) "reference detector". Similarly, the magnetized steel plate structure of the $10 \mathrm{kton}$ MINOS far detector is being designed in considerable detail, while at the same time alternative methods for fabricating the 8-meter wide octagonal steel plates are being investigated. Finally, the conceptual design of the MINOS electronics system has been started, including special electronics needed to read out RPC's and liquid scintillator detectors.

Argonne physicists and engineers are heavily involved in three separate aspects of the MINOS detector development program. First, the Argonne and Tufts groups are collaborating to optimize the design of the Iarocci chamber reference detector. This involves the development of mass production techniques, the choice of chamber materials 
and gas mixture, and the study of chamber operation in both limited-streamer and proportional modes. Second, the Argonne and Livermore groups are working together on the optimization of the steel and magnet designs. Argonne is performing detailed magnetic field calculations and is also developing an alternative, thin-laminate design for the fabrication of the magnetized steel plates. Third, Argonne physicists and engineers are collaborating with groups at Oxford and Oak Ridge in planning for the engineering and prototyping of MINOS electronics systems. Development work in all three areas began during 1995. MINOS detector R\&D work at Argonne is being partially supported by two Argonne LDRD grants during FY 1996.

\section{b. Iarocci chambers}

Iarocci chambers operated in limited streamer mode are used in the MINOS "reference detector", whose design is described in the MINOS proposal. Although similar detector systems have already been built and operated successfully in other large calorimeters, several design issues which are specific to MINOS remain unresolved:

1. Is calorimeter response adequate with nonflammable gas mixtures? The traditional gas used in Iarocci chambers contains $75 \%$ isobutane, which is probably unacceptable in the underground laboratory environment at Soudan. Nonflammable mixtures contain only $10 \%$ isobutane.

2. Is calorimeter response adequate if Iarocci chambers are operated in proportional mode? This is the "backup" technology if limited streamer operation proves to be unsatisfactory with nonflammable gas.

3. We must identify materials to replace the traditional PVC plastic which has been used to construct Iarocci chambers in the past. (PVC releases toxic fumes when burned.) Aluminum, ABS, and Noryl are being investigated as possible alternative materials.

During 1995 the Argonne group constructed and operated several small test chambers using aluminum and PVC "profile" extrusions. The profile extrusion forms the body of an 8-m long Iarocci-tube chamber, consisting of eight parallel, 1-cm square channels. Eight $100 \mu \mathrm{m}$ diameter anode wires are stretched down the centers of the. channels and the entire assembly is enclosed in a gas-tight "sheath". Test chambers were constructed using bare aluminum profiles as well as aluminum coated with graphite, which may eliminate after pulsing observed previously with aluminum Iarocci chambers. A simple gas mixer system was constructed and used to make gas mixtures containing $10 \%, 30 \%$ and $75 \%$ isobutane.

The MINOS Iarocci chambers will be operated with the wires at ground and the profiles at negative high voltage, to allow the anode wire signals to be read out without 
blocking capacitors. All large Iarocci chamber arrays which have been built previously have operated the anode wires at positive high voltage. Initial tests of prototype chambers at Argonne have shown that operation with grounded anode wires requires a conductive cover over the open side of the profile, in order to have efficient collections of ionization from the entire volume of the $1 \mathrm{~cm}$ square tubes. This cover must have a high resistivity to allow the detection of chamber signals on the external cathode strip plane. At the end of December, all prototype chambers were being modified to include an internal resistive cover. Studies of chamber performance with different gas mixtures and chamber materials will continue in 1996.

\section{c. Steel and magnet engineering}

The MINOS far detector consists of 600 toroidally magnetized steel plates, each of which is an 8-m wide, 4-cm thick octagon with a small central hole for the magnet coil. Planes of Iarocci chambers are located in the 3-cm wide gaps between the plates, which must therefore be quite flat. For solid steel plates, the toroidal field would be about 1.5 Tesla and would be quite uniform over most of the plate area. a low power $(50 \mathrm{~kW})$ conventional copper coil with a single return leg is sufficient to produce this field. In the reference design, the plates are assembled from 2-cm thick, 2-m wide steel strips up to 8$\mathrm{m}$ long, which are the largest pieces which can be moved down the Soudan mine shaft. The Livermore group is responsible for optimizing the engineering of this reference design.

Two alternative techniques are being considered for the construction of the steel plates. During 1995, Argonne physicists and engineers developed a conceptual design in which the 8-m octagonal plates are constructed from sixteen identical triangle-shaped pieces. Each of the sixteen pieces would be prefabricated at a commercial facility from seven 6-mm thick laminates, which are staggered to provide overlapped joints. A preliminary estimate of the fabrication cost of plates constructed in this way showed it to be similar to the cost of the reference design. This thin-laminate construction technique should produce very flat plates with small internal air gaps. The triangular pieces which must be moved underground are both smaller and lighter than the large steel slabs of the reference design.

The engineering of a second alternative design is being investigated at the University of Minnesota. This concept uses a spiral winding of long strips of thin, 4-cm wide steel bar stock around a central core. The 4-cm thick circular plates would be wound underground like reels of magnetic tape. The study of mechanical engineering issues and the development of cost estimates are just beginning for this design.

Initial magnetic field calculations for the MINOS proposal were performed by Larry Turner of the Argonne Advanced Photon Source magnet group, under the supervision of HEP Division physicists. Magnetic field simulation work continued 
throughout 1995 in order to evaluate field quality for different construction techniques. The effects of air gaps between component pieces of individual octagonal plates has become a particular concern as the magnetic models have become more realistic. Variations in the patterns of air gaps from plate to plate affect the predictability and uniformity of the magnetic field, and thus can degrade the momentum resolution. Plates with larger air gaps also require more coil power to achieve a given field strength. During 1995 the Argonne group also performed field measurements on small steel magnet models in order to check and calibrate the magnetic field calculations, and to evaluate the properties of different types of steel.

During 1996 the Argonne MINOS group will continue to work out the engineering details of the thin-laminate steel plate design. Issues include realistic manufacturing costs, flatness and mechanical stability, and the effect of air gaps on field strength and quality. The group will continue to make use of magnet engineering expertise in other Argonne divisions to develop realistic magnetic models of all three steel plate construction techniques. The program of field measurements on small magnet models will be completed in early 1996.

\section{d. Electronics}

Much of the MINOS electronics development work is being postponed as long as possible in order to take advantage of the most modern technology available at the time construction begins. Standard technology which is already in wide use is completely adequate for the MINOS reference detector design, however newer technology may result in improved capabilities and reduced costs. During 1995 the MINOS collaboration began work on the conceptual design of the data acquisition and triggering electronics in order to develop an initial plan for the electronics engineering and fabrication. This will ensure that funds for engineering effort, prototype construction, and production fabrication will be available at the appropriate times.

Alternative active detector technologies will have different electronics requirements, whose costs will be a factor in the final active detector choice. While electronics for Iarocci chambers and RPC's will have similar overall architectures, a liquid scintillator detector might be quite different, depending in the readout technique. For example, the triggering of a liquid scintiallator detector read out with image intensifiers would be entirely different from that already designed in detail for the reference detector. RPC's and scintillators also have the potential for fast timing which could proide time-of-flight measurements for cosmic ray events. The MINOS electronics group will design and build prototypes of specialized front-end electronics needed for tests of all active detector technologies in 1996.

While the Argonne electronics group devoted most of its effort in 1995 to such global system design issues, it also began initial work on specific circuits needed in the 
near term: front-end circuits to suppress after pulsing in Iarocci chambers and RPC's, techniques for obtaining fast timing information from scintillator and RPC signals, and new readout electronics for the Soudan 2 near detector at Fermilab. (There is not enough existing Soudan 2 electronics to operate both the Soudan 2 detector at Soudan and the 20module near detector which will be installed at Fermilab.) the group also constructed a prototype 8-m long cathode strip and used it to measure transmission-line characteristics and to study real cathode signals from a prototype Iarocci chamber. Work will continue in 1996 on the design of specialized circuits to support chamber prototype measurements, both in the laboratory and in test beam studies at SLAC and Fermilab.

\section{I.C.7 Electronics Support Group}

(D. Ayres)

Work continued in support of the Nucleon Decay Experiment, Soudan 2. Our involvement during the period was one primarily of construction and maintenance. We modified 48 Analog Card, repaired 3 Anode H. V. Distribution Boxes, repaired 1 Smart Fuse Box, repaired 1 Calibration Pulser Card, and repaired 15 Preamp Motherboards. In addition we designed and built 12 Volt Relay Imbalance Boxes, and designed and built 1 TASSO Voltage Distribution Chassis. Miscellaneous other pieces of electronic equipment were maintained as necessary. We spent considerable time working on the conceptual framework for triggering and data acquisition in the Long Baseline Detector, and cooperated in building and testing prototype hardware.

Historically, our major effort with regard to support of the ZEUS calorimeter was the development of the first level calorimeter trigger processor(CFLTP) and the trigger for the Small Angle Rear Tracker (SRTD). The Zeus calorimeter first-level trigger processor presents summary data on energy deposition in the uranium/scintillator sampling calorimeter to the global first-level trigger (GFLT). The summary data includes global and regional sums of electromagnetic and hadronic energy deposition, the number of isolated muons and isolated electrons, missing transverse energy, jet cluster information, and the likelihood of beam-gas background. The CFLTP receives data from 16 regional trigger pre-processors which digitize the calorimeter signals and perform regional energy sums and logical operations. Design and construction of these regional pre-processors is the responsibility of our collaborators from Wisconsin. During the period we produced, tested, and furnished to ZEUS 5 Input Cards to be used at DESY as spares.

A new trigger which we built was added to the ZEUS detector during the winter shutdown for use during the 1995 running period. The Small Angle Rear Tracking Detector (SRTD) has 272 scintillation fingers arranged as four quadrants in two overlapping planes around the beampipe and in front of the Rear Calorimeter. The scintillators are viewed by photomultipliers via fiber bundles. As photomultiplier outputs are carried to the data acquisition system, they are spied upon by the trigger. High impedance amplifiers, dual level discriminators, and coincidence logic furnish stops to 
four rapid cycling TDC's which are cleared by the HERA clock every $96 \mathrm{~ns}$. The trigger data furnished to the Global First Level Trigger by the SRTD on every beam crossing consists of 32 bits. One 8-bit word is generated for each SRTD quadrant. It represents the TDC time (6 bits) with a resolution of $1 \mathrm{~ns}$ and the position ( 2 bits) of the hit or hits closest to the beampipe. The trigger hardware includes programmable thresholds, delays, and circuitry to capture scintillator hit patterns and trigger times of interest. An extensive set of diagnostic and operational software has been developed. During the period we also redid the artwork for the EVB/FLT Card and produced, assembled, and tested 3.

We are beginning a heavy involvement with the Level 2 Trigger for the ATLAS Detector at the LHC at CERN. We expect that this work will lead to our having the responsibility in conjunction with our colleagues from Michigan State University for the Design and Construction of the Level 2 Trigger Supervisor and Region of Interest Builder. These are major efforts which will require effort for a number of years. During this period we built and provided to the ATLAS Test Beam at CERN a Prototype Supervisor Module which ran with hardware provided by our collaborators. We built a prototype R5900 Phototube Base for evaluation for the ATLAS Tilecal - the hadron calorimeter. We also are involved in the testbeam effort with regard to the Tilecal and during the period began a design and development effort to support these tests. This work included design of a charge injector system for the ADC's and work on the test module manipulator.

During FY 1992 and FY 1993 we have built and tested electronics for the CDF trigger upgrade. This was an effort to bring the preshower radiator and shower max detector wires into the trigger at second level to improve the efficiency for B physics, and in fact did improve the efficiency of triggering on B's by a factor of 3 . We hope to have a part in the upgrade to $132 \mathrm{~ns}$ operation, and during the period the study of noise problems continued. We expect to have a significant part in this upgrade in the areas of data acquisition from the shower max and preshower chambers and formulation of the trigger using shower max and tracking data.

During the period, we designed and built a Faraday Cup ADC to support the Advanced Accelerator Group, and also built and Air Cylinder Controller Chassis to support the SLAC testbeam hardware for the Profile Monitor project.

\section{(J. Dawson)}




\section{THEORETICAL PHYSICS PROGRAM}

\section{II.A THEORY}

\section{II.A.1 Lattice Formulation of Chiral Gauge Theories}

Over a course of several years, Geoffrey Bodwin has developed a method for formulating gauge theories of chiral fermions, such as the Standard Electroweak Model, on the lattice. (Recently, a similar proposal has been developed independently by Hernández and Sundrum.) The early stages of this work were carried out in collaboration with Eve Kovács (Fermilab).

There are two essential ingredients in the method that Bodwin proposes. The first is to replace the magnitude of the determinant for a fermion with chiral couplings to the gauge field with the square root of the determinant for the same fermion, but with vectorlike couplings to the gauge field. The second ingredient is to take a double limit in which the spacing of the lattice on which the fermion resides is sent to zero before the spacing of the lattice on which the gauge field resides. (The motivation for these ingredients is summarized in the report for January 1-June 30,1993.) A lengthy paper describing this chiral-fermion method [ANL-HEP-PR-95-59] was completed in October. The paper contains a proof, to all orders in perturbation theory, that the method yields a gaugeinvariant theory with the correct low-energy spectrum. The paper also contains an argument that the method is valid, configuration by configuration, even in the presence of nonperturbative, but bounded, gauge fields. It remains an open question as to whether the presence of large transformations of the gauge field, which have been shown by Shamir to lead to singularities on the fermion lattice, could invalidate the method.

(G. Bodwin)

\section{II.A.2 Top Quark Production Dynamics.}

Edmond L. Berger and Harry Contopanagos have been investigating the theoretical description of top quark production at hadron collider energies within the context of perturbative quantum chromodynamics (QCD). Their initial motivation was both phenomenological and theoretical. For some time it has been known that, especially near production threshold, the size of the QCD next-to-leading order term in the cross section exceeds that of the leading order term. Correspondingly, the important premise of perturbation theory that successive terms in the perturbative expansion should be decreasingly smaller is not valid for the production of heavy quarks near threshold, a region of phase space important for top quark production at the Fermilab Tevatron. The origin of the large threshold enhancement may be traced to initial-state soft-gluon radiation in the hard-scattering subprocess in which heavy quarks are generated. To 
obtain more reliable theoretical estimates of cross sections in such situations, the effects of gluon radiation must be reevaluated through a procedure known as soft-gluon resummation to all orders in the QCD running coupling strength. Berger and Contopanagos have carried out a complete calculation of soft-gluon resummation in both the quark-antiquark and gluon-gluon channels. They computed and have published the physical cross section for inclusive $t \bar{t}$ production as a function of top mass in protonantiproton reactions at center-of-mass energies 1.8 and $2.0 \mathrm{TeV}$. Their results at $1.8 \mathrm{TeV}$ are shown in Fig. 1 along with data from the CDF and D0 collaborations. Berger and Contopanagos summarized their calculation in several papers that have emerged during the second half of 1995. These include ANL-HEP-PR-95-31, published in Phys. Lett. B361 (1995) 115-120; ANL-HEP-CP-95-81, an invited paper presented at the International Europhysics Conference on High Energy Physics, Brussels; and ANL-HEPCP-95-85, (hep-ph/9512212) an invited paper presented at the International Symposium on Heavy Flavor and Electroweak Theory, Beijing. In a long paper nearing completion, [ANL-HEP-PR-95-82, to be submitted to Physical Review], Berger and Contopanagos provide a thorough exposition of their method of soft-gluon resummation. They derive the perturbative regime of the resummed series, starting from Principal Value Resummation and isolating the perturbative gluon radiation phase space upon inversion of the corresponding Mellin transform. They show explicitly that their perturbative result is independent of the manner Principal Value Resummation regularizes non-perturbative effects. They compare their approach in detail with another resummation method that relies on the choice of infrared cutoffs. They derive the scale-dependence properties of the resummed cross section, and they discuss factorization scheme dependence and remaining theoretical uncertainties, including possible non-perturbative contributions.



Figure 1. Calculation by Berger and Contopanagos of the total $t \bar{t}$ production cross section as a function of top mass, in the MSscheme, at $\sqrt{ } s=1.8 \mathrm{TeV}$. The upper and lower dashed lines denote the band of perturbative uncertainty, and the solid center line is the central-value prediction. The published CDF and D0 data are also shown.

(E. Berger, H. Contopanagos) 


\section{II.A.3 t-channel Unitarity Construction of Small-x Kernels}

In lectures presented at the XXXV Cracow School of Theoretical Physics [ANL-HEPCP-95-83], Alan White describes and extends recent work with Claudio Coriano. The BFKL equation is first presented as a reggeon Bethe-Salpeter equation and the use of reggeon diagrams to obtain 2-2 and 2-4 reggeon interactions at $O\left(\mathrm{~g}^{4}\right)$ is discussed. The dispersion theory basis of multiparticle $j$-plane analysis is elaborated and it is shown how a gauge theory can be studied by combining Ward identity constraints with the group structure of reggeon interactions. The derivation of gluon reggeization, the $O\left(g^{2}\right)$ BFKL kernel, and $O\left(g^{4}\right)$ corrections, is described within this formalism. An explicit expression for the $O\left(g^{4}\right)$ forward "parton" kernel is given in terms of logarithms and the eigenvalues evaluated. A separately infra-red finite component with a holomorphically factorizable spectrum is shown to be present and conjectured to be a new leading-order partial-wave amplitude. A comparison is made with Kirschner's discussion of $O\left(g^{4}\right)$ contributions from the multi-Regge effective action.

(A. White)

\section{II.A.4 Deep Inelastic Diffractive Scaling}

Presently, diffractive physics is thought to be non-perturbative and uncalculable within QCD, except in limited kinematic circumstances involving small-x and large $Q^{2}$, where it is hoped semi-perturbative BFKL Pomeron calculations will be applicable. Recently, however, Buchmüller has noticed a remarkably simple scaling relation between the diffractive and full deep-inelastic cross-sections measured at small-x at HERA. This scaling suggests an extraordinary simplicity for the Pomeron at large $Q^{2}$. In ANL-HEPPR-95-57, Alan White describes how an explanation for this simplicity is provided by his previous study of the Pomeron in QCD. He argues that the parton interpretation given by Buchmüller and Hebecker can be understood within QCD as the appearance of the Pomeron in a Super-Critical phase. In diffractive hard scattering, the Pomeron effectively appears as reggeized gluon exchange in a color-compensating background field. The formalism can also be applied to diffractive $\mathrm{W}$ production at the Tevatron. If the scaling is a true asymptotic property, then it should anticipate the appearance of a further massive sector of QCD and associated asymptotic Critical Pomeron behavior.

(A. White)

\section{II.A.5 Isolated Photon Cross Sections}

Edmond Berger, Xiaofeng Guo, and Jianwei Qiu (Iowa State University) have written a paper entitled "Breakdown of Conventional Factorization for Isolated Photon Cross Sections", Argonne report ANL-HEP-PR-95-88 (hep-ph/9512281), accepted for 
publication in Physical Review Letters. High energy photons have long been considered an excellent probe of short-distance physics in strong interactions. They couple directly to pointlike quark constituents and do not interact much once produced. For observational reasons the inclusive prompt photon cross section may not be measurable at high energy. Owing to backgrounds from, e.g., $\pi^{0} \rightarrow \gamma \gamma$, a single high energy photon is observed and the cross section is measured only when the photon is relatively isolated. A proper theoretical treatment of the cross section for isolated photons requires careful consideration of the origins and cancellation of both infrared and collinear singularities in QCD perturbation theory. In a theoretical calculation, isolation of the photon restricts the final-state phase space accessible to accompanying quarks and gluons. In their paper, using $e^{+} e^{-} \rightarrow \not X X$ as an example, Berger, Guo, and Qiu demonstrate that this phase space restriction inevitably breaks the perfect cancellation of infrared singularities between real gluon emission and virtual gluon exchange diagrams that is required to yield finite cross sections in each perturbative order. Breakdown of the cancellation of infrared singularities appears first at next-to-leading order in the fragmentation contributions. In isolated photon production, breakdown of factorization means that the cross section cannot be factored into a sum of terms each having the form of an infrared-safe partonic hard part times a corresponding parton-to-photon fragmentation function. This effect limits the region of $x \gamma=2 E \gamma / \sqrt{s}$ in which quark-to-photon fragmentation functions may be extracted reliably from electron-positron reaction data. For production of isolated photons at hadron-hadron colliders, the physical cross section is obtained after an integration over the momentum fractions of incoming partons. One is not free to impose a selection on $x \gamma$ analogous to that in $e^{+} e^{-}$annihilation, and the integration is done throughout the part of phase space where the breakdown of factorization takes place. It is therefore not altogether straightforward to specify the precise form and magnitude of the fragmentation contribution to isolated prompt photon production in hadron-hadron collisions. More discussion of this question will be found in a longer paper that Berger, Guo, and Qiu are now preparing

(E. Berger)

\section{II.A.6 Double Prompt Photon Production in Polarized Proton-Proton Scattering}

Claudio Coriano (Univ. of Florida) and Lionel Gordon completed a calculation of double prompt photon production in polarized and unpolarized proton-proton scattering $(p p \rightarrow y \gamma X)$ using both analytical and Monte Carlo methods. The calculation was done in next-to-leading order quantum chromodynamics (QCD), and at this stage represents only the third full next-to-leading order calculation performed for polarized processes. In their paper [ANL-HEP-PR-95-84], Coriano and Gordon briefly examine the possibility that double prompt photon production will be useful to supplement information from other sources on the polarized gluon distribution of the proton, $\Delta G$, about which very little experimental information is available. They are currently carrying out a more 
detailed phenomenological study of the process at energies appropriate for the BNL heavy ion collider RHIC, which is due to start taking data in a few years.

(L. Gordon)

\section{II.A.7 Lattice Measurement of Matrix Elements for Decays of Heavy Quarkonium}

Geoffrey Bodwin, Seyong Kim, and Don Sinclair are continuing work on a project to determine the nonperturbative operator matrix elements that appear in the factorization theorems for the decay rates of S-wave and P-wave charmonium and bottomonium systems. This work is based on the formalism for quarkonium decays developed by Bodwin, Braaten, and Lepage. The determination of the matrix elements entails two steps. First one measures the lattice-regulated matrix elements in a numerical simulation. (Details of this measurement are given in the Computational Physics section of this report.) Then one relates the lattice-regulated matrix elements to the continuumregulated (MS) matrix elements through a perturbative $\mathrm{QCD}$ calculation. The continuum matrix elements are the ones that appear in existing expressions for the quarkonium decay rates, and they are more physical than the lattice matrix elements, in that they scale with the heavy-quark velocity according to simple rules that follow from the nonrelativistic dynamics. (The lattice matrix elements contain spurious, power ultraviolet divergences that violate the simple velocity-scaling rules.)

Preliminary results for the matrix-element determination have now been worked out. The matrix elements that correspond to the square of the wavefunction at the origin, in the S-wave case, and the square of the derivative of the wavefunction at the origin, in the P-wave case, have been measured previously. The results of Bodwin, Kim and Sinclair are in good agreement with these previous determinations. On the lattice, the Swave matrix element of order $\mathrm{v}^{2}$ is dominated by spurious power ultraviolet divergences. These are, in principle, removed by the perturbative subtractions that relate the lattice matrix elements to the continuum ones. However, Bodwin, Kim, and Sinclair have found that the size of the divergent contributions leads to large errors in the remainder when one works to one-loop accuracy. The use of alternative definitions of the lattice matrix element to reduce the size of the power divergences is currently being explored. For the color-octet P-wave matrix element, preliminary results show reasonable agreement with a phenomenological analysis of the data for charmonium, with the bulk of the continuum. matrix element coming from the perturbative corrections. In the case of bottomonium, comparison of the lattice result for the color-octet matrix element with experiment will have to wait until the decay rate of the $\chi_{61}$ state into light hadrons has been measured.

(G. Bodwin) 


\section{II.A.8 Geometry and Duality in Supersymmetric Sigma-models}

Duality addresses nontrivial alternate descriptions of field theory (and strings). In the past [Phys. Rev. D52 (1995) R573], Cosmas Zachos and Thomas Curtright (Univ. of Miami) have introduced the Supersymmetric Dual Sigma Model (SDSM), a two-dimensional field theory constructed to be nonlocally equivalent to the supersymmetric Chiral $\sigma$ Model. This canonical equivalence, the first for a supersymmetric theory, was proven by virtue of a generating functional expressly invented for this purpose. The explicit maps discovered illuminate the nonlocality inherent in nonabelian duality.

In ANL-HEP-PR-95-90 [hep-th/9601096, published in Nucl. Phys. B469 (1996) 488], these authors, with Tsuneo Uematsu (Univ. of Kyoto), elaborate novel features of this theory (SDSM). This is achieved both by now formulating the model in superspace (thus configuring the formal dual transition in terms of superfields) and also by direct construction of the special chiral-entwined realization of supersymmetry which extends the bosonic DSM. As a consequence, the same model can now be seen to be reachable through three alternate paths.

The third pathway sheds light on the highly unconventional inhomogeneous geometry of the dual manifold, which is worked out explicitly here for the first time. This geometry is expected to underlie the resolution of a two-loop renormalizability conundrum recently encountered by Subbotin and Tyutin. The chiral-entwining encountered further elucidates the fermion-boson symphysis observed, i.e. the conjoining of bosonic and fermionic contributions to the bosons ("disorder pions") of the dual theory.

A third result of the project is a representation of canonical transformations in terms of Poisson Bracket (m.m. canonical commutator) invariance, instead of the less direct invariance of Hamilton's equations (popular in the standard literature), which naturally sets the stage for Dirac quantization.

The four results outlined could sit comfortably in separate publications. Nevertheless, in avoidance of serial publication, they may be appreciated more thoroughly under a common roof in this extensive paper on the full structure of the SDSM.

$$
\text { (C. Zachos) }
$$

\section{II.A.9 Production of a Prompt Photon in Association with a Heavy Quark}

Edmond Berger and Lionel Gordon concluded a fully analytic calculation of the inclusive production of a prompt photon in association with a heavy quark at large values of transverse momentum. This analysis was done at next-to-leading order in perturbative 
quantum chromodynamics (QCD). Their paper, [ANL-HEP-PR-95-36 (hepph/9512343)], was submitted to Physical Review D in December, 1995. In collaboration with Bob Bailey (Eckerd College), Berger and Gordon also began extending this work using a combination of analytic and Monte Carlo integration methods in order to obtain differential distributions, including photon isolation restrictions, that should facilitate contact with experimental results at hadron collider energies. Berger and Gordon show that the study of the two-particle inclusive distribution, with specification of the momentum variables of both the final prompt photon and the final heavy quark, tests correlations inherent in the QCD matrix elements and provides a means for measuring the charm quark density in the nucleon. Seven partonic subprocesses contribute at order $\alpha^{2}$. Berger and Gordon find important corrections to the lowest order, $O\left(\alpha_{s}\right)$, subprocess $c g \rightarrow \gamma$. For the interval in transverse momentum $p_{\mathcal{T}}^{\gamma}$ of current experimental interest, $10 \mathrm{GeV}<p_{\mathcal{T}}^{\gamma}<50 \mathrm{GeV}$, subprocesses initiated by $g c$ and $\not g$ scattering are the most important quantitatively at Fermilab Tevatron energies. Dominance of the subprocess initiated by $g c$ scattering is preserved after the next-to-leading terms are included, justifying use of data from $p+\bar{p} \rightarrow \gamma+c+X$ in attempts to measure the charm quark momentum density in the nucleon. Other subprocesses account for about 50\% of the cross section at currently accessible values of $p_{\mathcal{T}}^{\gamma}$. The "background" associated with these subprocesses must be taken into account in analyses done to extract the charm quark density.

$$
\text { (E. Berger, L. Gordon) }
$$

\section{II.A.10 Analysis of Recent Polarization Experiments}

Recently, polarized deep-inelastic scattering experiments, which were performed at SLAC and CERN have been analyzed. Gordon Ramsey and M Goshtasbpour (Center for Theoretical Physics, Tehran) have performed an analysis of the proton, neutron and deuteron data to extract parton information from the corresponding structure functions. Their study includes both the integrated structure functions and their Bjorken- $x$. dependence. They find that the polarized parton distributions (quark and gluon) are related to their corresponding unpolarized distributions by a flavor-dependent normalization factor. When this factor is extracted from the data for the integrated distributions, the corresponding $x$-dependent distributions fit the data very well. Their initial results will be published in the proceedings of the "VI International Workshop on High Energy Spin Physics", held in Protvino, Russia, in September [ANL-HEP-CP-95-71 and hep-ph-9512250]. Further work is underway to understand these results could lead to a suitable model for polarization. In conjunction with this work, suitable parametrizations of the flavor-dependent distributions as functions of $x$ and $Q^{2}$ are being generated. These are useful in calculations of spin-dependent observables.

(G. Ramsey) 


\section{II.A.11 Report of the TeV2000 Study Group on Future Electroweak Physics at the Tevatron}

Steve Mrenna contributed to this effort to quantify the collider physics potential of a future Tevatron. The physics program entails searches for physics beyond the Standard Model. One of the new physics scenarios that must be confronted is the possibility of a Supersymmetric origin to electroweak symmetry breaking. Such a scenario introduces a host of supersymmetric partners to the Standard Model particle spectrum, all with masses from a few hundred $\mathrm{GeV} / \mathrm{c}^{2}$ to a $\mathrm{TeV} / \mathrm{c}^{2}$. All of those particles which are kinematically accessible to the Tevatron should be produced with cross sections of the same size as other standard Electroweak processes. In addition, there should be a Higgs boson with a mass less than $150 \mathrm{GeV} / \mathrm{c}^{2}$. Previous and current work on Higgs boson physics and Supersymmetry signals at the Tevatron was largely incorporated into the Light Higgs Physics and Supersymmetric Physics chapters of the report.

(S. Mrenna)

\section{II.A.12 High-pT Higgs Boson Production at Hadron Colliders to $O\left(\alpha_{s} G_{F}^{3}\right)$}

Because of the large top-quark Yukawa coupling, electroweak radiative corrections to Higgs boson production can be large. Steve Mrenna and Chien-Peng Yuan (Michigan State Univ.) [hep-ph/9507235 (accepted for publication in Phys. Rev. D)] studied high$p_{T}$ Higgs boson = production in hadron collisions at one-loop to order $O\left(\alpha_{s} G_{F}^{3}\right)$. In particular, the electroweak contributions to the process $g+q(q) \rightarrow q(q)+H$ where $q=u, d, c, s$, or $\sigma$, for the LHC (a $\sqrt{s}=14 \mathrm{TeV}$, proton-proton collider) were compared to the QCD radiative corrections at $O\left(\alpha_{s}^{3} G_{F}\right)$ and the tree level $O\left(\alpha_{s} G_{F}\right)$ calculations. The associated production of a high- $p_{\tau}$ Higgs boson with a 6 -quark or antiquark at $o\left(\alpha_{s} G_{F}^{3}\right)$ is comparable to the $O\left(\alpha_{s}^{3} G_{F}\right)$ and $O\left(\alpha_{s} G_{F}\right)$ processes because of the large top quark mass and the additional contribution of electroweak gauge and Goldstone bosons. The associated production of light quarks, however, is not significant. Because of the equivalence theorem relating Goldstone bosons and longitudinally polarized $\mathcal{W}$-bosons for large energies, it was also possible to investigate new physics effects in the framework of the electroweak chiral Lagrangian. They found that the electroweak radiative corrections are particularly sensitive to new physics which modifies either the $t-b-W, t-t-H$, or $\mathcal{W}-W-H$ vertices.

(S. Mrenna) 


\section{II.A.13 Detecting a Light Stop from Top Decays at the Tevatron}

Supersymmetric models which attempt to explain the apparent discrepancy between the experimental values of $\alpha_{s}\left(\mathcal{M}_{z}^{2}\right) R_{b}$ and $\mathcal{A}_{L R}$ obtained from LEP/SLC data predict a light mass for the lightest top squark. In ANL-HEP-PR-95-66 (accepted for publication in Phys. Lett. B), Steve Mrenna and Chien-Peng Yuan (Michigan State Univ.) considered the possibility of discovering or excluding a light top squark (stop) $\tilde{4}_{1}$ based on top quark decays in the $\bar{t}$ events produced at the Fermilab Tevatron. In particular, they considered the Minimal Supersymmetric Standard Model with the sparticle spectrum

$$
m_{\chi_{1}^{ \pm}}+m_{b}, M_{W}+m_{x_{1}^{0}}+m_{b}>m_{l_{1}}>m_{\chi_{1}^{0}}+m_{c}
$$

where $\chi_{1}^{0}$ is the lightest neutralino, so that $t \rightarrow \tilde{t}_{1} \chi_{1}^{0}$ and $\tilde{t}_{1} \rightarrow c \chi_{1}^{0}$. All other sparticle masses were assumed to be heavier than $m_{t}$. Using this decay mode, the present search limits for a light top squark can be extended.

(S. Mrenna)

\section{II.A.14 Diffractive Deep Inelastic Scattering and Exclusive Vector Meson Production at HERA in Perturbative QCD}

Diffractive deep inelastic scattering has attracted a lot of interest since the first Large Rapidity Gap events were reported by $\mathrm{HI}$ and ZEUS. The existence of large rapidity gaps can only be explained by a color neutral exchange known as the "Pomeron" (Pomeranchuk Pole): any color exchange leads to a shower of partons which immediately fills the gap. To understand the nature of the Pomeron, whether it is hard, soft, or it undergoes a transition from hard to soft depending on the scales of the process, which is more likely, is a central problem and may find clarification in analysis of the new HERA data. The final state, which is generically an open hadronic system, may eventually become a vector meson, when the mass is taken to be in the resonance region. Both processes, Photon Diffractive Dissociation and Vector Meson production in Deep Inelastic Scattering, are closely related and should be studied in the same context. The simplest QCD model for the Pomeron is the two-gluon color singlet exchange, which couples to the hadronic (partonic) final state with preservation of gauge invariance. Including pairwise interactions between the two gluons leads to the hard (or BFKL) Pomeron, associated with the strong rise of the cross section at increasing energy. The complete answer, however, is only reached by considering unitarity corrections. They become stronger when the soft region is entered, transforming the hard into a soft Pomeron. By virtue of the $k_{t}$-factorization theorem, the two-gluon coupling to the hadronic final state should be a good approximation despite the complexity of corrections affecting the Pomeron structure. The remainder of the process including the hadronic final state is perturbatively calculable. 
In collaboration with Jochen Bartels (Hamburg Univ.), John Ellis (CERN), and Henrik Kowalski (DESY), Mark Wüsthoff is developing a parametrization for Diffractive Deep Inelastic Scattering, displaying the main features which result from the use of perturbative QCD. It is designed to provide a simple interpretation and to allow a direct comparison with oncoming data.

In the case of Vector Meson production at large momentum transfer, the Pomeron is perturbatively calculable. Together with Jochen Bartels, Hans Lotter (Hamburg Univ.) and Jeff Forshaw (Univ. of Manchester) Mark Wüsthoff has completed a paper discussing Vector Meson production at large $t$ [ANL-HEP-PR-95-89]. The exact solution for the BFKL-equation for nonzero momentum transfer was used and a small shrinkage found. The cross section was calculated and found to be large enough to be measurable at HERA.

(M. Wüsthoff)

\section{II.B COMPUTATIONAL PHYSICS (LATTICE GAUGE THEORY)}

The computational physics effort is devoted to numerical simulations of lattice quantum field theories, primarily of lattice quantumchromodynamics (QCD). The lattice provides the needed ultraviolet regulation of the theory and allows numerical simulations, which are the only reliable way of calculating non-perturbative results from $\mathrm{QCD}$. This enables one to investigate the behaviour of hadronic/nuclear matter at finite temperature and/or finite baryon number density, and, in particular, to study the transition to a quark-gluon plasma. These calculations have relevance to the physics of the early universe, to neutron stars, and to relativistic heavy ion collisions (RHIC). In addition, lattice QCD enables us to calculate basic properties of hadrons, such as their masses and decay rates.

For some time we have been involved in a project aimed at calculating the matrix elements which describe the decays of charmonium and bottomonium into light hadrons, in collaboration with G. T. Bodwin of the theory group. We have now calculated the matrix elements describing the decays of S- and P-wave bottomonium at $6 / \mathrm{g}^{2}=6.0$, and charmonium at $6 / \mathrm{g}^{2}=5.7$ (both quenched) on the lattice. For our larger lattice calculations $\left(16^{3} \times 32\right)$ we have used the CRAY C-90 at NERSC. The perturbative coefficients relating these lattice matrix elements to their continuum counterparts have been calculated. Preliminary results have been calculated and indicate that, within the limitations of our calculations (lowest order in $\mathrm{v}^{2}$; one loop perturbation theory), we can expect good agreement with experimental measurements.

We have been calculating the masses of flavour singlet mesons, in particular the $\eta^{\prime}$ and the $\sigma\left(f_{0}\right)$, in collaboration with J. B. Kogut (University of Mllinois), and their screening masses at finite temperature (Fig. 1). So far we have worked in the quenched approximation at both zero and finite temperature. These are of interest since they have a 


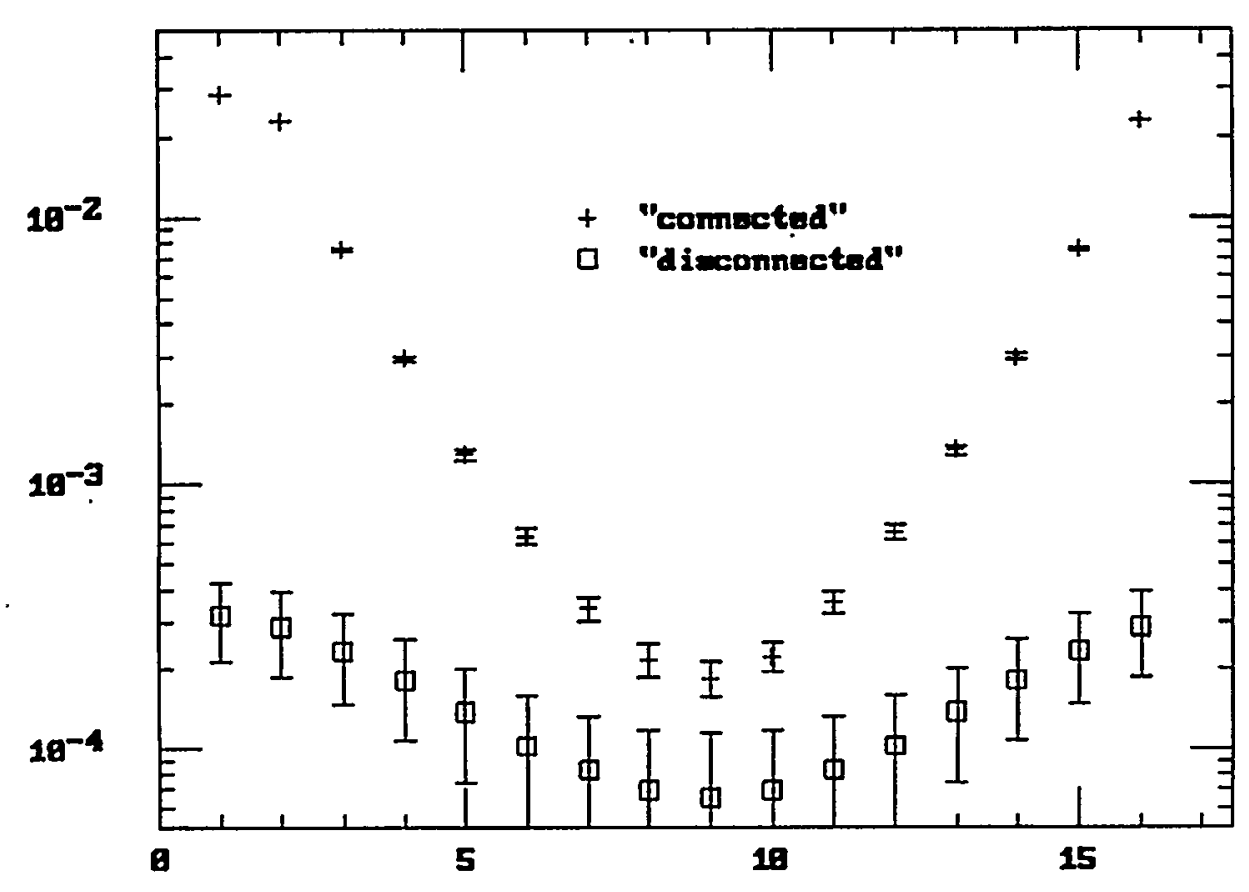

Fig 1. Propagator for the disconnected contribution to the $\eta^{\prime}$ propagator.

contribution from mixing with multi-gluon states. Because of this, the $\eta^{\prime}$ is sensitive to topological excitations (instantons, monopoles, etc.). Thus, at non-zero temperature, these calculations can be used to monitor the role of topological excitations in the transition from hadronic matter to a quark-gluon plasma. These studies are also using the CRAY C-90 at NERSC.

One of us (JFL) is extending the work he started at the University of Kentucky which calculates various matrix elements (in particular those with disconnected contributions) on the lattice. These include matrix elements which describe the partition of energy and angular momentum between the constituents in hadrons. The latter matrix elements include those related to the lowest non-trivial moments of the spin-dependent structure functions. These are of considerable interest experimentally since they are related to the so-called proton spin crisis.

Finally we have started on a project with J. B. Kogut which modifies the lattice QCD lagrangian by adding a chiral 4-fermion interaction. Such a term is a higher dimensional (irrelevant) operator, which should not modify the physics of QCD. However, such a term changes the behavior of the Dirac operator, such that it no longer is 
is singular for zero quark mass. This makes it possible to simulate near and even at the chiral $\left(\mathrm{m}_{\mathrm{q}}=0\right)$ limit, without paying the price of having to invert a nearly singular matrix, which requires large amounts of computer time.

(D. K. Sinclair, J. F. Lagae) 


\section{ACCELERATOR RESEARCH AND DEVELOPMENT PROGRAM}

\section{III.A ARGONNE WAKEFIELD ACCELERATOR PROGRAM}

During this reporting period, AWA activities focused on commissioning the main "drive" linac and on completing the high brightness witness beam electron gun and its beam transport system. Demonstration that the potent drive beam pulses required for the generation of $100 \mathrm{MeV} / \mathrm{m}$ accelerating wakefields were achievable was a major step in the AWA program.

\section{III.A.1 AWA Facility Status}

\section{a. Drive Beam Gun and Linac}

As previously reported, we had observed electron yields from the gun which, although very intense, were highly non-linear with respect to the intensity of laser illumination of the cathode. We analyzed the time structure of the beam with a streak camera and confirmed that at high laser intensity each laser pulse resulted in a "train" of electron pulses spaced at the $1.3 \mathrm{GHz}$ rf period (fig 1.). This was a clear signature of "explosive mode" emission, a phenomena not well understood but observed by other photocathode based gun developers.

When operated below this threshold the quantum efficiency, $\mathrm{QE}$, of both copper and magnesium was substantially less (by a factor of ten or so) than that

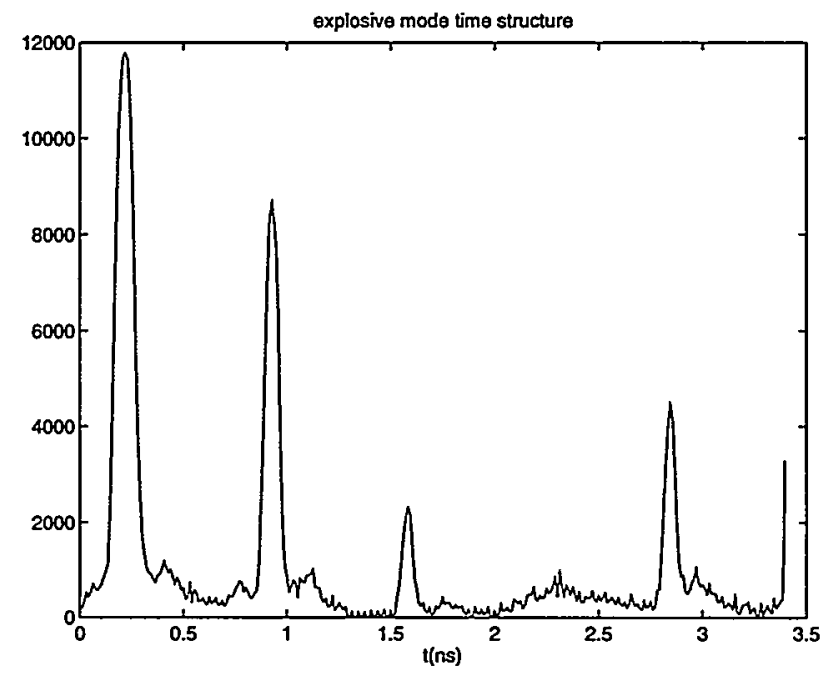
available in the literature on which we based the design parameters of the AWA. In an effort to find a better cathode material, we Figure 1 systematically measured the quantum efficiency of pure copper, magnesium, calcium, yttrium, and "glidcop", a matrix of aluminum oxide and copper. We were unable to find any metallic material with quantum efficiency better than that of magnesium. Responding to reports that photo-emission occurred preferentially at grain boundaries, we tested copper whose surface had been "shot-peened" to produce abundant dislocations. 
By scanning a small laser spot on the cathode we searched for possible "hot spots" to no avail.

Finally, we discovered that some improvement in $\mathrm{QE}$ was possible by very careful surface preparation followed by a hexane rinse. Why hexane is particularly effective is not clear, but it works.

Electron Pulse Length vs Charge

Our measured magnesium $\mathrm{QE}$ is still a factor of five or so from the published "best" value, but is consistent with that quoted by the vast majority of gun research groups.

In the fall of 1995 we systematically measured the drive beam properties. We confirmed that the AWA drive beam is a unique among photo-cathode driven sources by virtue of its high current and short pulses capability (see Figure 2). We anticipate modest increases in performance (e.g. shorter pulse lengths) will be obtained when we begin operation with the specially

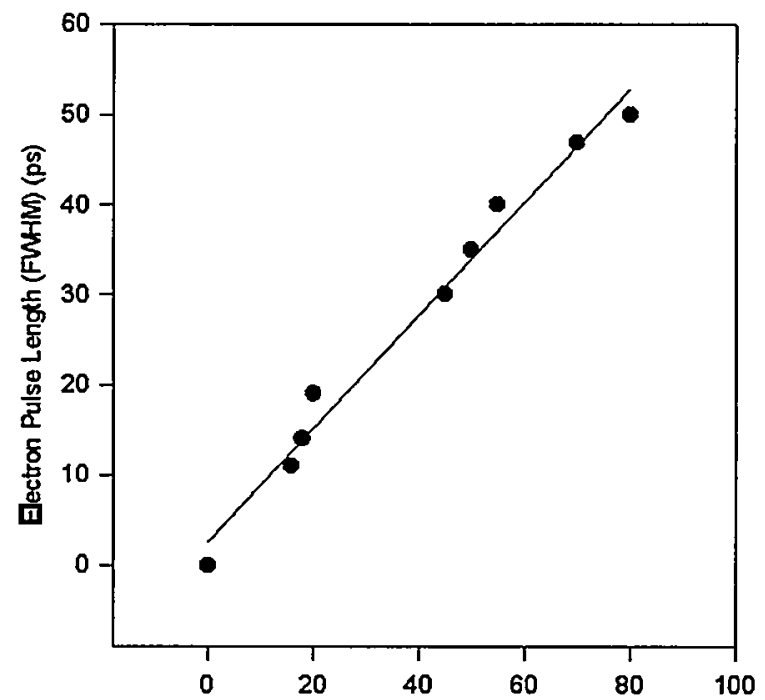

Figure 2 shaped laser pulses in late spring, 1996. It should be noted however, that the present beam is already sufficient for all but a few of our planned wakefield experiments.

Late summer saw a shut-down of the AWA during which we made several modifications and improvements.

1. New cooling tubes were installed in the drive beam linac tanks and a new paint job added for good looks.

2. An adjustable tuning stub was installed in the gun, eliminating the need to position the cathode as a means to tune the structure and solving some difficulties with arcing in the gun.

3. Non-destructive intensity monitors were installed to compliment Faraday cup monitors.

4. The AWA shield vault was enlarged to accommodate more research area. 


\section{b. Witness Gun System}

The 6-cell witness gun cavity was completed and tuned. Upon return to Argonne after being brazed we discovered rather severe vacuum leaks in the braze joints between the cells. For expediency we decided to repair these leaks at ANL rather than return them to the brazing company.

The gun and the beam line elements used to transport the witness pulses to and combine them with the drive pulses have been mounted on the support tables. Alignment and final installation details will be completed during the first two months of 1996.

\section{III.A.2 Experiments and New Ideas}

\section{a. Dielectric Based Experiment}

A short $(30 \mathrm{~cm})$ section of dielectric loaded, specially instrumented wave guide was installed and briefly studied. The purpose was to validate the techniques we plan to use to measure the forward flow of multi-Ghz beam induced rf power in such a wave guide. The test was successful, confirming values we had predicted.

\section{b. Plasma Wakefield Experiment}

By reflecting the laser pulse from a mirror which had a "recessed" section in the middle of the laser spot, we produced a pseudo witness pulse along with a drive pulse in the main AWA gun. This combined drive-witness beam was then directed into the UCLA plasma cell for some preliminary tests. The results were encouraging, but various problems such a beam clipping in the plasma cell entrance and (at that time) relatively long pulses limited the success. Nevertheless, it was a useful experiment in that it identified areas needing modification and improvement.

\section{c. New Ideas}

We are fabricating an X-band dielectric device as a possible NLC structure. We intend to test this at SLAC this spring. Dielectric devices have significant advantages as rf-driven accelerators as well as wakefield accelerators. The suppression of multibunch beam breakup is well understood and is easier to implement than in conventional iris loaded copper structures. Undesirable space harmonics of the accelerating field are also absent in the dielectric device.

We have begun investigating laser-driven structures, which offer the possibility of using the high fields available in a laser pulse to provide very high gradients for particle acceleration. 
One such technique involves the use of a radially polarized laser such as the one available at Brookhaven for use in inverse Cherenkov acceleration experiments. We have proposed a method to efficiently couple laser radiation into a dielectric structure-- in fact the accelerating TM0n modes are identical to those of dielectric wakefield structures. This allows acceleration over extended distances and within vacuum, clear advantages over the inverse Cherenkov schemes proposed thus far. Gradients in excess of $500 \mathrm{MeV} / \mathrm{m}$ should be possible using this method, and parasitic wakes can be controlled using the same mode suppression techniques developed for dielectric wakefield devices. A preliminary proposal has been presented for an experimental test of this concept at BNL-ATF.

Another laser accelerator under study involves planar dielectric structures. These structures consist of two dielectric layers separated by a vacuum gap through which the electron beam propagates. Laser light is coupled into the structure via partially reflecting mirrors. The dielectric constant (or alternatively the thickness) of the dielectric is modulated along the direction of propagation of the electron beam at the laser wavelength; the standing wave excited in the structure by the laser pulse is then synchronous with the electron bunch. One very attractive aspect of these devices is that for deflecting wakefields due to beam misalignments are small. Plans for experiments with these structures at the Argonne Wakefield Accelerator and at the Brookhaven Accelerator Test Facility are being developed.

(J. Simpson)

\section{III.B HIGH RESOLUTION PROFILE MONITOR DEVELOPMENT}

The apparatus has now been installed in the Final Focus Test Beam at SLAC and we are beginning to use beam to tune up and align the system. The vacuum chambers, computer, and all the mechanical and electrical components arrived at SLAC in the first week of September. Following unpacking and trial assembly, the chambers and hardware were cleaned and vacuum checked. Then they and the collimator supports were mounted in the beamline along with a number of new components in the E144 systems aligned with a transit, connected to the vacuum and pumped down. The collimator insertion and retraction systems were installed and checked out along with the encodermotors which constituted the precision collimator control. This is shown in Figure 1.

In addition to the collimators, a periscope was designed and constructed of $1 / 8^{\prime \prime}$ steel. This box contained the pair converter target, an air Cherenkov radiator, periscope mirrors and mount for the camera. One mirror of the periscope was remotely adjustable in two dimensions using encodermotors, which permitted remote alignment of the whole detector system. The camera and power supply had been operated in March; however a new computer was installed to permit control of more encodermotors and other peripherals. Programming changes in LabWindows were done during the summer by 
Amit Patel, an undergraduate. The control computer, printer, monitor, CCTV monitor, CAMAC and Pneumatic/Hydraulic control panel were rack mounted in Building 406.

The primary problems in this experiment were expected to be alignment and backgrounds. Neither limited performance or speed of setup during the initial runs. We got our first parasite beam on December 13, and were immediately able to determine that the image intensifier used in the camera was capable of a dynamic range of greater than a million, effectively permitting us to measure the divergence of the electron beam directly, and through a nine radiation length absorber (CCM1). We were also able to align the detector periscope. This parasite beam was followed by a few minutes of primary beam, when we were able to sequentially insert each collimator and verify that a strong signal was detected thru each gap.

When we got additional time in December, we were able to insert the primary collimator and the upper slit, do some preliminary alignment using beam and some initial scans of the primary collimator (with a 50 micron gap) and the upper slit (which had a 200 micron gap) against each other. These showed that comparatively large motions ( 0.3 $\mathrm{mm}$ ) of the collimators could be easily made to adjust to the set of tunes and configurations of the electron and photon beams. Fig. 2 shows profiles of the detected signal at the pair converter, showing low levels of background. Fig. 3 shows the result of scans of the upper slit and collimator against each other. Since the slit was not closed, this data does not measure beam profiles. We expect to make high resolution beam profile measurements in the summer of 96.

(J. Norem) 


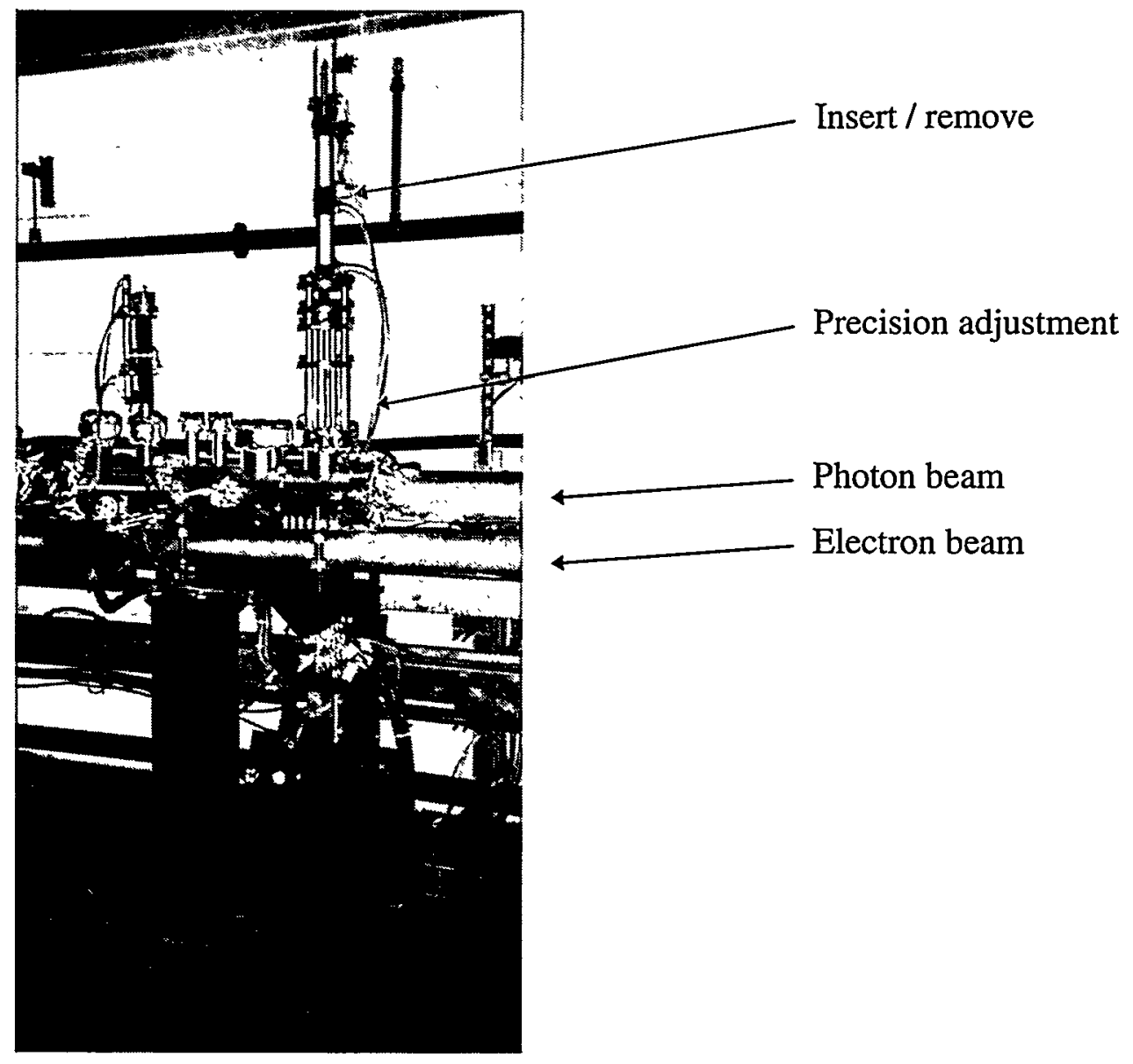

Figure 1. The primary collimator in the photon line. The electron beam is below it.

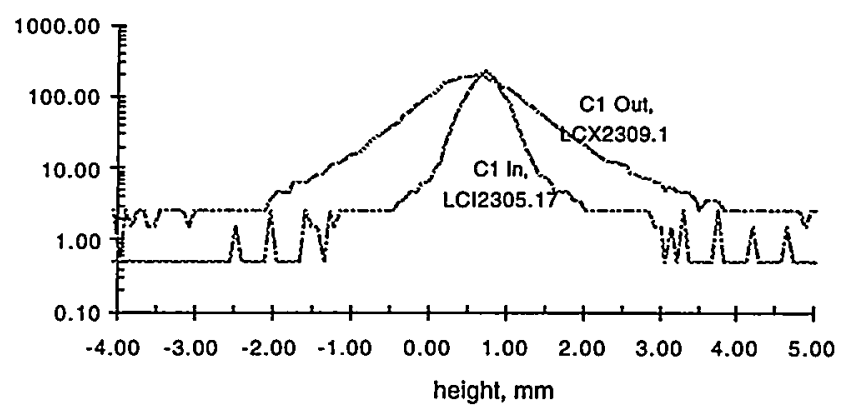

Figure 2. Detected signals in the pair converter as a function of height off the median plane.
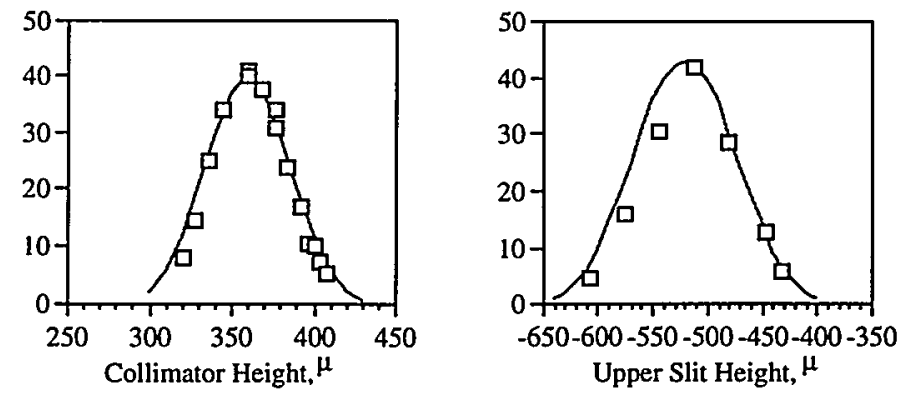

Figure 3. Scans of the collimator and upper slit against each other. 


\section{DIVISIONAL COMPUTING ACTIVITIES}

\section{IV.A HIGH PERFORMANCE COMPUTING: THE PASS PROJECT}

\section{IV.A.1 R\&D During the Period July 95 thru December 95}

Two physicists (L. Price and E. May) and a computer scientist (D. Malon) from DIS division continued to work on the "Petabyte And Storage Solutions" (PASS) project. This is a HPCC and HEP supported R\&D project to study the use of database technologies for the storage of and access to scientific data on the scale of a few petabytes ( $10^{15}$ bytes). Future HEP experiments will collect data at the rate of 1 petabyte per year; new advanced technology (both hardware and software) is required to provide the access to this quantity of data in a fast and efficient manner for a world-wide HEP environment. This work is being done in collaboration with the University of Ilinois at Chicago (UIC), University of Maryland, and Lawrence Berkeley lab (LBL). I describe briefly some PASS activities in which ANL staff had the principal role or made important contributions.

We made a detailed investigation of the OMG CORBA technology for distributed object management, in particular the Persistence Services Protocol. Prepared a design suitable for the PASS architecture and implemented a demo using the IBM SOM/DSOM Corba product for AIX.

We made a detailed investigation of the ODMG standard version 1.1 for objectoriented databases. We determined the object model and the API advocated by the ODMG group are suitable for scale and type of data to be managed by the PASS achitecture. We developed a $\mathrm{C}++$ code to implement sufficient portions of the ODMG C++ API which allows us to build ODMG compliant object-oriented databases on top of the PASS light-weight object manager. As a test of this technology we implemented a persistent object data model for a standard HEP monte carlo code ISAJET. The data from standard ISAJET runs was generated, loaded into the model and stored in the hierarchical I/O and mass storage system of the ANL/MCS IBM SP1 parallel computer. Studies of ODMG complient queries using parallel $\mathrm{I} / \mathrm{O}$ access to the stored data have been initiated. Initial results for an 8 server system have yielded thru puts of typically 32 to $48 \mathrm{MB} / \mathrm{sec}$ for the NSL Unitree file system and the IBM PIOFS parallel disk system, respectively.

We prepared talks and papers for the 1995 Computing in HEP conference. We initiated a collaboration with the RD45 project (A Persistent Object Manager for HEP) which is doing R\&D work in preparation for CERN Large Hadron Collider experiments.

(E. May) 


\section{PUBLICATIONS}

\section{A. JOURNAL PUBLICATIONS, CONFERENCE PROCEEDINGS, BOOKS}

A Possible Method to Produce a Polarized Antiproton Beam at Intermediate Energies

H. Spinka, E. W. Vaandering and J. S. Hofmann

Proceedings of the Eighth International Symposium on Polarization Phenomena in Nuclear Physics, editors E. J. Stephenson and S. E. Vigdor, American Institute of Physics Conference Proceedings \#339, p. 713, Woodbury, NY (1995)

Analyzing Power Measurement in Inclusive Lambda-0 Production with a $200 \mathrm{GeV} / \mathrm{c}$ Polarized Proton Beam

A. Bravar, et al

Phys. Rev. Lett. 75.3073 (1995)

Breakdown of Conventional Factorization for Isolated Photon Cross-Sections Edmond L. Berger, Xiafeng Guo, Jianwei Qis

Phys. Rev. Lett. 76, 2234-2237 (1996)

Canonical Nonabelian Dual Transformations in Supersymmetric Field Theories

T. Curtright, C. Zachos

Phys. Rev. D. 52, R573, (1995)

Decay Rates of Various Bottomonium Systems

Seyong Kim

Lattice 1995:437-440 (QCD 161:1715:1995)

Detecting a Light Stop from Top Decays at the Tevatron

S. Mrenna (ANL), C. P. Yuan

Phys. Lett. B $\underline{367}$, 188-194 (1996)

Exclusive $\rho^{\circ}$ Production in Deep Inelastic Electron-Proton Scattering at HERA

By ZEUS Collaboration (M. Derrick et al)

Phys. Lett. B $\underline{356}$, 601-616 (1995)

Gauge Theory High-Energy Behavior from J Plane Unitarity

C. Coriano, A. R. White

Nucl. Phys. B468, 175 (1996)

Geometry and Duality in Supersymmetric $\sigma$ - Models

T Curtright, T Uematsu, and C Zachos

Nucl. Phys. B 469 488-512 (1996) 
Inclusive Charged Particle Distributions in Deep Inelastic Scattering Events at HERA By ZEUS Collaboration (M. Derrick et al)

Z. Phys. C므, 1-16 (1996)

Inclusive Prompt Photon Production in Hadronic Final States of Electron-

Positron Annihilation

Edmond L. Berger (ANL), Xiao-Feng Guo, Jian-Wei Qiu

Phys. Rev. D프, 1124 (1996)

Light Hadron Masses with 4-GeV Cutoff and L = 2.4 FM

Seyong Kim (ANL), Shigemi Ohta

Lattice 1995: 350-353 (QCD 161:1715:1995)

Light Hadron Spectroscopy

D. K. Sinclair

Lattice 1995: 112-127 (QCD 161:1715: 1995)

Long-Baseline Neutrino Oscillation Experiments

D. Crane and M. Goodman

Proceedings of the 1994 Snowmass Summer Study, Particle and Neuclear

Astrophysics and Cosmology in the Next Millenium, p. 225, World

Scientific, 1995, E. Kolb and R. Peccei, editors

Measurement of Alpha-S from Jet Rates in Deep Inelastic Scattering at HERA

By ZEUS Collaboration (M. Derrick et al)

Phys. Lett. B $\underline{363}, 201-216$ (1995)

Measurement of Elastic $\rho^{\circ}$ Photoproduction at HERA

By ZEUS Collaboration (M. Derrick et al)

Z. Phys. C무, 39-54 (1995)

Measurement of correlated $\mu-\bar{b}$ jet cross sction in $p \bar{p}$ collisions at $\sqrt{s}=1.8 \mathrm{TeV}$

By CDF Collaboration

Phys. Rev. D $\underline{53}, 1051$ (1996)

Measurement of Single Spin Asymmetry for Direct Photon Production in pp Collisions at $200-\mathrm{GeV} / \mathrm{c}$

By E704 Collaboration (D. P. Grosnick, D. L. Adams, D. A. Hill et al (ANL)

Published in Proceedings of the Eleventh International Symposium on High

Energy Spin Physics, K. J. Heller and S. L. Smith, editors, American Institute of Physics Conference Proceedings \#343, p. 404, Woodbury, NY (1995) 
Measurement of the B Meson and B Quark Cross-Sections at S** $(1 / 2)=1.8 \mathrm{TEV}$ F. Abe et al

Phys. Rev. D $\underline{50}, 4252$ (1995)

Measurement of the Cross Section for the Reaction $\partial \rho \rightarrow \ni / \psi \rho$ with the ZEUS Detector at HERA

M. Derrick and the ZEUS Collaboration

Phys. Lett. B $\underline{350}, 120$ (1995)

Measurement of the Proton Structure Function F 2 at Low x and Low $Q^{2}$ at HERA

M. Derrick et al

Z. Phys. Cㅌ9, 607-620 (1996)

Measurement of the Ratio $\sigma \mathrm{B}(p \bar{p} \rightarrow W \rightarrow e v) / \sigma B\left(p \bar{p} \rightarrow Z^{\circ} \rightarrow e e\right)$ in $p \bar{p}$ collisions at $\sqrt{s}=1800 \mathrm{GeV}$

L. Nodulman

Phys. Rev. D. V프, 2624 (1995)

Measurement of the W Boson Mass

F. Abe et al

Phys. Rev. D $\underline{52}, 4784$ (1995)

Measurement of the W Boson Mass

F. Abe et al

Phys. Rev. Lett. $\underline{75}, 11$ (1995)

Neutrino Oscillation Experiments with Atmospheric Neutrinos

T. Gaisser, M. Goodman

Proceedings of the 1994 Snowmass Summer Study, Particle and Neuclear

Astrophysics and Cosmology in the Next Millenium, p. 220, World Scientific, 1995, E. Kolb and R. Peccei, editors.

Neutron-Proton Elastic Scattering Spin-Spin Correlation Parameter Measurements Between 500 and $800-\mathrm{MeV}$. 3. Mixtures of C(SS), C(LS), C(LL), and C(NN).

V. Carlson, R. Garrett, D. Hill (ANL)

Phys. Rev. D. 53, 3506-3533 (1996)

New Results in Nucleon-Nucleon Scattering at Intermediate Energies

H. Spinka

Proceedings of the Eighth International Symposium on Polarization Phenomena in Nuclear Physics, editors E. J. Stephenson and S. E. Vigdor, American of Physics Conference Proceedings \#339, p. 275, Woodbury, NY (1995) 
Partial Siberian Snake Experiment at the AGS

H. Huang et al (M. Beddo, D. Grosnick, D. Lopiano, H. Spinka, D. G. Underwood,

A. Yokosawa [ANL])

Proceedings of the Eleventh International Symposium on High Energy Spin

Physics, editors K. J. Heller and S. L. Smith, American Institute of Physics

Conference Proceedings \#343, p. 90, Woodbury, NY (1995)

Perturbative Gluon Resummation of the Top Quark Production Cross Section

Edmond L. Berger, Harry Contopanagos

Phys. Lett. B361, 115-120 (1995

Properties of High-Mass Multijet Events at Fermilab Proton-Antiproton Collider

L. Nodulman

Phys. Rev. Lett. $\underline{75}$, 608 (1995)

Properties of the Scale Invariant $\mathrm{O}\left(g^{4}\right)$ Lipatov Kernel

A. R. White, C. Coriano

Nucl. Phys. B451, 231 (1995)

Quantum Mechanical Time-Development Operator for the Uniformly Accelerated Particle

R. W. Robinett

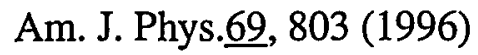

Quenched QCD at Finite Density

J. Kogut, M. Lombardo, D. K. Sinclair

Phys. Rev. D $\underline{1}$, 1282-1291 (1995)

Rapidity Gaps Between Jets in Photoproduction at HERA

M. Derrick et al (By Zeus Collaboration)

Phys. Lett. B $\underline{369}$, 55-68 (1996)

Scale Invariant $\mathrm{O}\left(\mathrm{g}^{4}\right)$ Lipatov Kernels at Nonzero Momentum Transfer

C. Coriano, R. R. Parwani, A. R. White

Nucl. Phys. B $\underline{468}, 219$ (1996)

Search for New Particles Decaying to Dijets in $p \bar{p}$ Collisions at $\sqrt{s}=1.8 \mathrm{TeV}$

L. Nodulman

Phys. Rev. Lett., 74, 3538 (1995)

Search for Squarks and Gluinos via Radiative Decays of Neutralinos in ProtonAntiproton Collisions at $\sqrt{s}=1.8 \mathrm{TeV}$

L. Nodulman

Phys. Rev. Lett., $\underline{75}, 613$ (1995) 
Single-Spin Asymmetries and Invariant Cross Sections of the High Transverse Momentum Inclusive pi-0 Production in $200 \mathrm{GeV} / \mathrm{c}$ pp and pbarp Interactions

D. L. Adams et al (D. P. Grosnick, D. A. Hill, et al [ANL])

Published in Proceedings of the Eleventh International Symposium on High Energy Spin Physics, K. J. Heller and S. L. Smith, editors; American Institute of Physics Conference Proceedings \#343, p. 422, Woodbury, NY (1995)

Spin Physics at E-704 and RHIC

David G. Underwood

Proceedings of the Workshop on the Prospects of Spin Physics at HERA, p. 49-54, Zeuthen, Germany (1995), edited by J. Blumlein and W. D. Nowak

Studies of Hadron Electron Separators for the ZEUS Barrel Calorimeter

I. Ambats, D. Bortz, A. Connolly, A. Derlicki, M. Derrick, W. Kahle, S. Magill, D. Mikunas, B. Musgrave, J. Schlereth, R. Stanek, J. Thron

Nucl. Instrum. Meth. A $\underline{368}, 364-377$ (1996)

t-Channel Unitarity Construc̀tion of Small-x Kernels

Claudio Coriano, Alan R. White Acta Phys. Polon. B26, 2005-2038 (1995)

The Argonne Wakefield Accelerator High Current Photocathode Gun and Drive Linac P. Schoessow, E. Chojnacki, G. Cox, W. Gai, R. Konecny, J. Power, M. Rosing, J. Simpson, N. Barov, M. Conde

Proceedings of the 1995 Particle Accelerator Conference and International Conference on High-Energy Accelerators, Vol. 2, 97.9 (1995)

The Electric Charge of a Dirac Monopole at Nonzero Temperature C. Coriano, R. Parwani

Phys. Lett. B363, 71 (1995)

The Movable Polarized Target as a Basic Equipment for High-Energy Spin Physics Experiments at the JINR Dubna Accelerator Complex

F. LoNar et al (D. Grosnick, D. Hill, T. Kasprzyk et al [ANL])

Nucl. Instrum. Methods A 356 , 58-61 (1995)

The Spectrum of the $\mathrm{O}\left(\mathrm{g}^{4}\right)$ Scale Invariant Lipatov Kernel

A. White

Phys. Rev. Lett. 74, 4980 (1995) 
The ZEUS Calorimeter First Level Trigger

S. Silverstein, I. Ali, B. Behrens, C. Foudas, C. Fordham, A. Goussiou, M. Jaworski,

J. Lackey, D. Reeder, P. Robl, W. H. Smith, A. Vaicuilis, M. Wodarczyk, J. Dawson,

D. Krakauer, R. Talaga, J. Schlereth, H. Zhang

Nucl. Instrum. Meth. A $\underline{360}$, 322-324 (1995)

Ultrahigh Energy.Cosmic Ray Composition from Surface Air Shower and Underground Muon Measurements at Soudan 2

N. P. Longley, et al

Phys. Rev. D $\underline{2}, 2760$ (1995)

Visualizing the Solutions for the Circular Infinite Well in Quantum and Classical Mechanics

R. W. Robinett

Am. J. Phys. 64, 440-446 (1996)

Witness Gun for the Argonne Wakefield Accelerator

J. Power, J. Simpson, E. Chojnacki, R. Konecny

Proceedings of the 1995 Particle Accelerator Conference and International

Conference on High-Energy Accelerators, Vol. 2 , 976 (1995)

Workshop on the Tau/Charm Factory

Ed. by J. Repond

American Institute of Physics, Conference Proceedings $\underline{349}, 518$ (1995)

B. PAPERS SUBMTTED FOR PUBLICATION IN JOURNAL/CONFERENCE PROCEEDINGS

A Lattice Formulation of Chiral Gauge Theories

G. T. Bodwin

ANL-HEP-PR-95-59

Submitted to Phys. Rev. D

Analytic Calculation of Prompt Photon Plus Associated Heavy Flavor at Next-to-Leading Order in QCD

Edmond L. Berger, L. E. Gordon

ANL-HEP-PR-95-36

To be published in Phys. Rev. D

Argonne Tau-charm Factory Collider Design Study

L. C. Teng et al

Proceedings of the Workshop on the Tau/Charm Factory, ed. J. Repond, AIP Conference Proceedings 349, American Institute of Physics, Woodbury, New York 
Buchmuller Scaling, The QCD Pomeron, and Tevatron Diffractive Hard Scattering Alan R. White

ANL-HEP-PR-95-57

Submitted to Phys. Rev.

Data Analysis in an Object Request Broker Environment

David Malon, Edward N. May

ANL-HEP-CP-95-70

Submitted to Proceedings of Computing in High Energy Physics

Decay Rates of Various Bottomonium Systems

S. Kim

ANL-HEP-CP-95-65

Submitted to Proceedings of the International Symposium on Lattice Field

Theory (LATTICE '95)

Diffractive Production of Vector Mesons at Large t

J. Bartels, J. R. Forshaw, H. Lotter, M. Wusthoff

Submitted to Phys. Lett.

Higher Order Corrections to BFKL Evolution from t-channel Unitarity

Claudio Coriano, Alan R. White

Presented at VI International Conference on Elastic and Diffractive

Scattering, Blois Workshop (1995)

Large Transverse Momentum Jet Production and the Gluon Distribution Inside the Proton J. Huston, E. Kovacs, S. Kuhlmann (ANL), H. L. Lai, J. F. Owens, D. Soper, W. K. Tung

Measurement of the Differences in the Total Cross Section for Antiparallel and Parallel Longitudinal Spins and a Measurement of Parity Nonconservation with Incident Polarized Protons and Antiprotons at $200 \mathrm{GeV} / \mathrm{c}$

D. P. Grosnick, D. A. Hill, T. Kasprzyk, et al (ANL)

Submitted to Phys. Rev. D

Measurement of the Total Cross-Section Difference Delta(Sigma-L) in N P Transmission at $1.19,2.49$ and $3.65 \mathrm{GeV}$

B. P. Adiacevich et al (D. P. Grosnick, D. A. Hill, et al [ANL])

Submitted to Z. Phys. C 
Measurement of the W Mass at CDF

L. Nodulman

ANL-HEP-PR-95-60

Submitted to Proceedings of the International Europhysics Conference on High

Energy Physics

Muon Colliders

J. Norem et al

To be published in the Proceedings of the 9th ICFA Workshop on Advanced

Dynamics and Technology Issues for mu+mu- Colliders (MUMU 95)

New Particle Searches at CDF

L. Nodulman (For the Collaboration)

ANL-HEP-CP-95-56

Submitted to Proceedings of the International Europhysics Conference on High

Energy Physics

Object Database Standards, Persistence Specifications, and Physics Data

David M. Malon (ANL), Edward N. May (ANL), Robert L. Grossman,

Christopher T. Day, David R. Quarrie

ANL-HEP-CP-95-82

Submitted to Proceedings of Computing in High Energy Physics

Parton Distributions from SMC and SLAC Data

Grdon P. Ramsey, M. Gostasbpour

ANL-HEP-CP-95-71

Submitted to Proceedings of the 6th Workshop on High Energy Spin Phenomena (Spin 95)

Pathologies of Quenched Lattice QCD at Nonzero Density and Its Effective Potential D. K. Sinclair

To be published in Phys. Rev. D.

Perturbative Resummation of Gluon Radiation for Top Quark Production

Edmond L. Berger, $\mathrm{H}$. Contopanagos

ANL-HEP-CP-95-51

To be published in the Proceedings of the Iowa State University Workshop on

Top Quark Physics

Polarized and Unpolarized Double Prompt Photon Production in Next-to-Leading Order QCD

L. E. Gordon

ANL-HEP-PR-95-84

Accepted by Nucl. Phys. B (In Press) 
Production of a Prompt Photon in Association with Charm at Next-to-Leading Order in QCD

B. Bailey, Edmond L. Berger (ANL), , L. E. Gordon (ANL)

ANL-HEP-PR-95-87

Submitted to Phys. Rev. D

Proton-proton Interactions Using the RHIC Polarized Collider

A. Yokosawa

Submitted to Proceedings of the Adriatico Research Conference on "Trends in Collider Spin Physics"

Proton-proton Interactions Using the RHIC Polarized Collider

A. Yokosawa

Submitted to Proceedings of the XII International Seminar of High Energy

Physics Problems

Resummation of Gluon Radiation and the Top Quark Production Cross-Section Edmond L. Berger, Harry Contopanagos

ANL-HEP-CP-95-81

Submitted to Proceedings of International Europhysics Conference on High

Energy Physics (HEP 95)

Single Spin Asymmetries in Inclusive Charged Pion Production by Transversely Polarized Antiprotons

A. Bravar et al (D. P. Grosnick, D. Lopiano, Y. Ohashi, et al [ANL])

Submitted to Phys. Rev. D.

Spin Physics at E-704 and RHIC

D. G. Underwood

ANL-HEP-CP-96-10 and DESY 95-200

To be published in proceedings of the Workshop on the Prospects of Spin Physics at HERA, p. 49-54, Zeuthen, Germany, 1995, edited by J. Blumlein and W-D Nowak, August 1995

The High-Energy Behavior of the Forward Scattering Parameters: An Amplitude Analysis Update

A. R. White (ANL), M. M. Block, B. Margolis

Presented at VI International Conference on Elastic and Diffractive Scattering

Blois Workshop (1995)

The Perturbative Resummed Series for Top Quark Production in Hadron Reactions

Edmond L. Berger, Harry Contopanagos

ANL-HEP-PR-95-82

To be published in Phys. Rev. D 
The Soudan 2 Detector. The Design and Construction of the Tracking Calorimeter Modules

W. W. M. Allison et al

Accepted for publication in Nuclear Instruments and Methods in Physics

Research A

Top Quark Production Dynamics in QCD

Edmond L. Berger, Harry Contopanagos

ANL-HEP-CP-95-85

Submitted to Proceedings of International Symposium on Heavy Flavor and Electroweak Theory, Beijing,, China

\section{TECHNICAL NOTES}

An Optical Model for the Prototype Module Performance from Bench Measurements of Components and the Test Module Response to Muons

J. Proudfoot, R. Stanek

TileCal No. 066

Experiences with Stacking the First Four ATLAS Submodules at Argonmne

N. Hill et al

ANL-HEP-TR-96-14

Numerical Calculation of the Sampling Variation Intrinsic to the TileCal Scintillator Geometry

C. Padilla, J. Proudfoot

TileCal No. 073

Procedure for Stacking Tile-Cell Submodules Using the Argonne Designed Stacking Fixture

N. Hill

ANL-HEP-TR-95-54

Report of "Time Saver" Surface Finish Tests on Czech Steel Sampler

N. Hill

ANL-HEP-TR-95-55

CDF Note 3265 Top Physics at the Tevatron

D. Amidei, P. Baringer, A. Heinson, S. B. Kim, T. LeCompte,

T. Liss, K. Tollefson, S. Vejcik, B. Winer, D. Winn 
CDF Note 3267

CDF Note 3289

CDF Note 3294

CDF Note 3310

CDF Note 3319

CDF Note 3328

CDF Note 3329

CDF Note 3342

CDF Note 3347

CDF Note 3350

CDF Note 3434

CDF Note 3435

CDF Note 3443
Measurement of the B- and B0-Bar Meson Lifetimes Using

Semileptonic Decays

F. Ukegawa, A. B. Wicklund

A Technique for Measuring the B Quark Cross Section at $630 \mathrm{GeV}$ T. LeCompte

The Bias in the Top Mass Sample Due to Hadronic TAU Decays M. Hohlmann

Limits on WWZ and WWG Couplings...Updated to Include Run $1 \mathrm{~A}$ and $1 \mathrm{~B}$

T. A. Fuess, C. H. Wendt

Results of Some Parametric Trigger Tracker Studies

T. LeCompte, G. Berchiesi

New Particle Searches at CDF

L. Nodulman, The CDF Collaboration

Measurement of the W Mass at CDF

L. Nodulman, The CDF Collaboration

Will we Find the Higgs in Run II?

S. Kuhlmann

Search for the FCNC Radiative Decays T to C Gamma and T to $\mathrm{U}$ Gamma

T. LeCompte, R. Roser

Proposal for a Straw Tube Replacement of the CDF Central Tracking Chamber

R. Blair, K. Byrum, V. Guarino, N. Hill, S. Kuhlmann,

T. LeCompte, J. Proudfoot, L. Nodulman, A. B. Wicklund, et al.

Further Update on B Meson Lifetime with Semileptonics

F. Ukegawa, A. B. Wicklund

Measurement of B- and B0 Lifetimes with Semieptonic Decays F. Ukegawa, A. B. Wicklund

Tree-Level Calc. of Assoc. Prod. of Stand. Model Higgs in $\mathrm{W}->\mathrm{WH}, \mathrm{Z}->\mathrm{ZH}$

M. Hohlmann 
CDF Note 3448 Layout of the Axial and Stereo Superlayers for the CST

V. Guarino, R. Wagner

CDF Note $3453 \quad$ Updates of Photon $+D^{\wedge *}+$ Cross Section

R. Oishi, S. Kuhlmann, B. Flaugher

CDF Note 3459 Status Report to the Fermilab PAC on the CDF Central Straw

Tracking Chamber

The CDF Collaboration

CDF Note $3461 \quad$ Observation of Top Dileptons with Hadronically Decaying Tau

Leptons

M. Gallinaro, M. Hohlmann

NuMI-B-92 Status Report on the Technical Design Report for NuMI Beam

J. Morfin et al

NuMI-L-100 Fermilab Answers to HEPAP Subpanel Supplemental Questions

FNAL NuMI Group and D. Ayres et al (MNNOS Collaboration)

NuMI-L-105 Minutes of the MINOS Collaboration Meeting at Fermilab,

July 10-11, 1995

D. Ayres

NuMI-L-111 Conceptual Design of the Front Concrete Section of the Far Reference Detector

V. Guarino

NuMI-112 Tau Neutrino Cross Sections

H. Gallagher and M. Goodman

NuMI-B-113 K-long Production Angle in the NuMI Beam

D. Crane

NuMI-L-116 Minutes of the MINOS Executive Committee Meeting of

October 1995

M. Goodman

NuMI-L-118 Transparencies from the MINOS Collaboration Meeting at Fermilab, October 13-14, 1995

D. Ayres

NuMI-L-119 Minutes of the MINOS Collaboration Meeting, October 1995

D. Ayres 
NuMI-L-120 Minutes of the MINOS Technical Board Meeting, October 1995

D. Ayres

NuMI-B-121 The Length of the NuMI Shield

D. Crane and M. Goodman

NuMI-L-130 MINOS Detector R\&D Plan

D. Ayres (The MINOS Collaboration)

NuMI- B-132 NuMI Position Paper: The Length of the NuMI Shield

D. Crane et al

NuMI-L-133 Transparencies from the MINOS Collaboration Meeting at

Fermilab, December 9-10, 1995

D. Ayres (ANL); M. Schub

NuMI-L-135 Minutes of the MINOS Executive Meeting, December 8, 1995 M. Goodman

NuMI-L-136 Minutes of the MINOS Collaboration Meeting at Fermilab, December 9-10, 1995

D. Ayres

NuMI-L-137 Minutes of the MINOS Technical Board Meeting at Fermilab, December 10, 1995

D. Ayres

NuMI-L-138 Minutes of the MINOS Detector R\&D Committee Meeting at Fermilab, December 8, 1995

D. Ayres

NuMI-L-153 Minutes and Summary of a MINOS Electronic Meeting at Argonne, December 11, 1995

J. Thron

PDK-619 Soudan 2Experiment Quarterly Status Report, April-June 1995 D. Ayres

PDK-621 Minutes of the July CEV Meeting R. Seidlein

PDK-623 Measurement of the Precision and Accuracy of the Event Time Reconstruction R. Seidlein (ANL); J. Carlson 
PDK-624 Soudan 2 Experiment Quarterly Status Report, July-September 1995 D. Ayres

PDK-625 Decisions of the Soudan 2 Collaboration Meeting at Argonne, October 15-17, 1995

D. Ayres

PDK-626 Neutrino Cross Sections

H. Gallagher; M. Goodman

PDK-627 Minutes of the CEV Meeting at Minneapolis

M. Goodman; R. Seidlein

PDK-629 Soudan 2 Experiment Quarterly Status Report, October-December 1995

D. Ayres

STAR 242 Jet Quenching Using Gamma-Jet Events, T. J. Lecompte and D. G. Underwood

STAR 245 A Study of Effects of Phi Cracks in the STAR EMC, K. Krueger, D. G. Underwood and T. Lecompte

Wakefield Note - WF-177

An Inverse Cherekov Accelerator Using a Dielectric Channelled Waveguide, July 1995, W. Gai and J. D. Simpson 


\section{COLLOQUIA AND CONFERENCE TALKS}

Edmond L. Berger,

"Resummation of Gluon Radiation and the Top Quark Production Cross-Section International Europhysics Conference on High Energy Physics (HEP 95), Brussels, Belgium (July 1995)

"Top Quark Production Dynamics in QCD"

Invited review at the Institute for High Energy Physiocs, Beijing. International Symposium on Heavy Flavor and Electroweak Theory, (August 1995)

Geoff Bodwin

"A Lattice Formulation of Chiral Gauge Theories"

Physics Department, Ohio State University (November 1995)

"A Lattice Formulation of Chiral Gauge Theories"

Physics Department, University of Illinois, Chicago (November 1995)

"A Lattice Formulation of Chiral Gauge Theories"

ANL/HEPD Theoretical Physics Seminar (November 1995)

M. C. Goldman

"Long-Baseline Neutrino Oscillation Experiments"

Workshop on Massive Neutrinos, Santa Fe, New Mexico (July 1995)

"Status of the Soudan 2 Experiment"

Astrophysics Seminar, Los Alamos National Laboratory, Los Alamos,

New Mexico (July 1995)

"Status of the Fermilab NuMI Project"

CERN to Gran Sasso Long-baseline Neutrino Oscillations Workshop,

L'Aquila, Italy (October 1995)

"Near/Far Beam Differences for A Long-baseline Neutrino Experiment"

CERN to Gran Sasso Long-baseline Neutrino Oscillations Workshop,

L'Aquila, Italy (October 1995)

\section{J. Nodulman}

"Top and Electroweak Physics at CDF"

Presented at MPI, Munich, Germany (July 1995)

"W Mass Measurement at CDF:

Presented at the International Europhysics Conference (Parallel Session), Brussels, Belgium (July 1995) 
"New Particle Searches at CDF"

Presented at the International Europhysics Conference (Parallel Session),

Brussels, Belgium (July 1995)

"Measuring the Top Quark, at Fermilab"

Illinois State University, Normal, Illinois (September 1995)

"Top and Electroweak Physics at CDF"

University of Utah (October 1995)

\section{K. Sinclair}

"Light Hadron Spectroscopy"

Presented at LATTICE 1995, Melbourne, Australia (July 1995)

\section{G. Underwood}

"Capabilities of STAR for Spin Physics"

Presented at RHIC Spin Review, Brookhaven National Laboratory (July 1995)

"Spin Physics at E-704 and RHIC"

Presented at the Workshop on the Prospects of Spin Physics at HERA, Zeuthen, Germany (August 1995)

\section{A. R. White}

"Buchmuller Scaling, Diffractive W Production, and the QCD Pomeron"

ANL-HEP Lunch Seminar (July 1995)

"Cosmic Rays and Forward Physics"

Workshop on Forward p-p Physics, CERN, Switzerland (August 1995)

"QCD with Sextet Quarks"

Seminar, CDF QCD Working Group (August 1995)

"Hard Diffraction: From HERA to the Tevatron"

CTEQ Workshop on Collider Physics, Michigan State University (October 1995)

\section{A. Yokosawa}

"RHIC Spin Physics"

Physics Department, Wayne State University, Detroit (July 1995)

"Collider Spin Physics at RHIC and STAR"

Adriatico Research Conference on "Trends in Collider Spin Physics",

University of Trieste, Trieste, Italy (December 1995) 


\section{Zachos}

"Nonlocal Canonical Equivalence of Field Theories, Nonabelian Duality, and Symphysis" University of Washington, (November 1995) 


\section{HIGH ENERGY PHYSICS COMMUNITY ACTIVITIES}

\section{D.S.Ayres}

Acting Project Manager and Deputy Spokesman for MINOS Project.

\section{$\underline{\text { E. Berger }}$}

Member, High Energy and Nuclear Physics Advisory Committee, Brookhaven National Laboratory, 1995-1998.

Member, Past Chairman, Committee on Meetings, American Physical Society, 1991Present. Appointed by the President of APS to propose guidelines and rules for the organization and operation of all meetings of the APS and its subunits. The major issue before the Committee at present is the implementation of plan for a truly general meeting involving all subunits of the Society.

Member, Organizing Committee, U.S. ATLAS Physics Workshop, Argonne National Laboratory, October 1995.

Member, past-Chairman, Argonne National Laboratory Director's Review Committee, Individual Investigator Laboratory Directed Research and Development Program, 19941996.

Member, Scientific Program Committee, XXXI Rencontre de Moriond, "QCD and High Energy Hadronic Interactions", Les Arcs, France, March 1996.

Member, International Advisory Committee, Snowmass Summer Study, Division of Particles and Fields of the American Physical Society, Snowmass, CO, June-July 1996

Member, Steering Committee, 11th Topical Workshop on Hadron Collider Physics, Padova, Italy, May 1996.

Organizing Committee, Sixth Conference on the Intersections between Particle and Nuclear Physics, May 1997.

\section{Maury Goodman}

Secretary, Fermilab User's Executive Committee Secretary, MINOS Executive Board 
E. May

Member, ESnet Remote Conferencing Working Group

\section{J. Nodulman}

Member, TeV2000 working group, subgroup on electroweak physics of CDF.

\section{Underwood}

Technical Director, STAR EMC

Member of Technical Committee for STAR experiment.

\section{Zachos}

Member, Editorial Board of Journal of Physics A: Mathematical and General 


\title{
VIII HIGH ENERGY PHYSICS DIVISION RESEARCH PERSONNEL
}

\author{
L. Price \\ Administration \\ Accelerator Physicists \\ W. Gai \\ Accelerator Physicists \\ J. Norem \\ P. Schoessow \\ J. Norem \\ Experimental Physicists \\ D. Ayres \\ R. Blair \\ K. Byrum \\ D. Crane \\ M. Derrick \\ T. Fields \\ T. Fuess \\ M. Goodman \\ D. Krakauer \\ S. Kuhlmann \\ T. LeCompte \\ S. Magill \\ E. May \\ D. Hill \\ J. Simpson \\ B. Musgrave \\ L. Nodulman \\ J. Proudfoot \\ J. Repond \\ R. Seidlein \\ H. Spinka \\ R. Stanek \\ R. Talaga \\ J. Thron \\ D. Underwood \\ R. Wagner \\ A. B. Wickland \\ A. Yokosawa
}
E. Berger
G. Bodwin
H. Contopanagos
C. Coriano
L. Gordon
J. F. Legae

Theoretical Physicists

Engineers, Computer Scientists, and Applied Scientists

\section{J. Dawson}

V. Guarino

W. Haberichter

N. Hill

S. Mrenna

D. Sinclair

A. White

M. Wusthoff

C. Zachos

J. Nasiatka

J. Schlereth

$X$. Yang
I. Ambats
L. Baika
H. Blair
G. Cox
D. Jankowski

Technical Support Staff

C. Allgower

N. Barov

M. Bei

H. Gallagher

A. Hardman

M. Hohlmann

Laboratory Graduate Participants

B. Bailey (Theory)

Visiting Scientists

M. Conde (AWA)

T. Curtright (Theory)

H. Huant
D. Mikunas
J. Okrasinski
J. Power
H. Zhang

T. Kasprzyk

L. Kocenka

D. Konecny

R. Rezmer
A. Raczka (Theory)
G. Ramsey (Theory) 